\title{
Total Synthesis and Structural Revision of the Marine Macrolide Neopeltolide
}

\author{
Daniel W. Custar, Thomas P. Zabawa and Karl A. Scheidt* \\ Department of Chemistry, Northwestern University, \\ 2145 Sheridan Road, Evanston, Illinois 60208

\section{Supporting Information}

\section{Table of Contents}

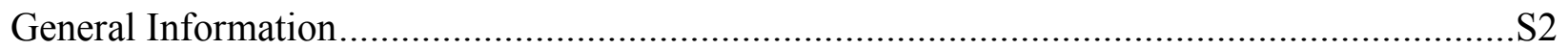

Experimental Procedures and Characterization Data for Synthesis of Proposed Structure.........S2

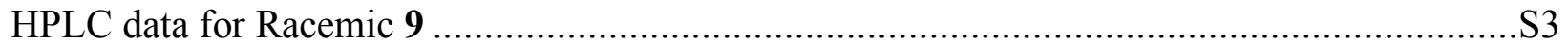

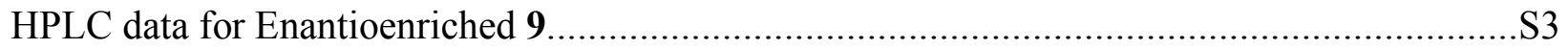

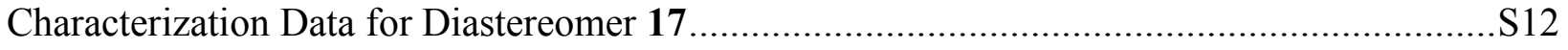

Experimental Procedures and Characterization Data for Synthesis of Revised Structure..........S12

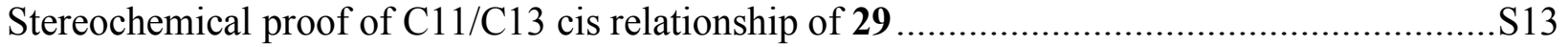

Comparison of ${ }^{13} \mathrm{C}$ Spectral Data of Isolated Neopeltolide, 1, and 21 .................................S20

Comparison of ${ }^{1} \mathrm{H}$ Spectral Data of Natural Neopeltolide and Synthetic Neopeltolide (21)......S21

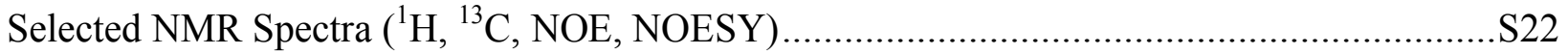




\section{General Information}

All reactions were carried out under a nitrogen atmosphere in flame-dried glassware with magnetic stirring. THF was purified by passage through a bed of activated alumina. ${ }^{1}$ Reagents were purified prior to use unless otherwise stated following the guidelines of Perrin and Armarego. ${ }^{2}$ Purification of reaction products was carried out by flash chromatography using EM Reagent silica gel 60 (230-400 mesh). Analytical thin layer chromatography was performed on EM Reagent $0.25 \mathrm{~mm}$ silica gel 60-F plates. Visualization was accomplished with UV light and ceric ammonium nitrate stain, anisaldehyde, or potassium permangenate stain followed by heating. Infrared spectra were recorded on a Perkin Elmer 1600 series FT-IR spectrometer. Optical rotations were measured on a Perkin Elmer Model 341 polarimeter with a sodium lamp and are reported as follows: $[\alpha] \lambda_{\mathrm{T}}{ }^{\circ} \mathrm{C}(\mathrm{c}=\mathrm{g} / 100 \mathrm{~mL}$, solvent $) .{ }^{1} \mathrm{H}$ NMR spectra were recorded on a Varian INOVA $500(500 \mathrm{MHz})$ spectrometer and are reported in ppm using solvent as an internal standard $\left(\mathrm{CDCl}_{3}\right.$ at $\left.7.26 \mathrm{ppm}\right)$. Data are reported as $(\mathrm{s}=$ singlet, $\mathrm{d}=$ doublet, $\mathrm{t}=$ triplet, $\mathrm{q}=$ quartet, $\mathrm{m}=$ multiplet, $\mathrm{b}=$ broad. All coupling constant(s) are reported in Hz. Protondecoupled ${ }^{13} \mathrm{C}$ NMR spectra were recorded on Varian INOVA $500(125 \mathrm{MHz})$ or INOVA 400 $(100 \mathrm{MHz})$ spectrometers and are reported in ppm using solvent as an internal standard $\left(\mathrm{CDCl}_{3}\right.$ at $77.0 \mathrm{ppm}$ ). Mass spectra data were obtained on a Varian 1200 Quadrupole Mass Spectrometer and Micromass Quadro II Spectrometer.

\section{Experimental Procedures and Characterization Data for Synthesis of Proposed Structure}

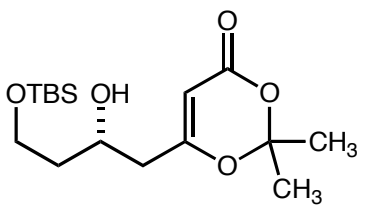

(S)-6-(4-(tert-butyldimethylsilyloxy)-2-hydroxybutyl)-2,2-dimethyl-4H-1,3-dioxin-4-one (9): A mixture of $(R)$-BINOL $(0.26 \mathrm{~g}, 0.9 \mathrm{mmol}), \operatorname{Ti}(i-\mathrm{OPr})_{4}(271 \mu \mathrm{L}, 0.9 \mathrm{mmol}), 4 \AA$ molecular sieves $(2.11 \mathrm{~g})$, and THF $(11 \mathrm{~mL})$ was stirred vigorously at room temperature under $\mathrm{N}_{2}$ for 60 minutes to yield a heterogeneous orange solution. The mixture was cooled to $-78{ }^{\circ} \mathrm{C}$ and a solution of aldehyde $7(1.14 \mathrm{~g}, 6.0 \mathrm{mmol})$ in THF $(24 \mathrm{~mL})$ was added via cannula and the resulting solution stirred for $30 \mathrm{~min}$. Enol silane 8 (2.61 g, $12.2 \mathrm{mmol})$ was added dropwise to the solution and the mixture was stirred vigoursly for 2 hours at $-78{ }^{\circ} \mathrm{C}$. The mixture was then warmed to $23{ }^{\circ} \mathrm{C}$ and allowed to stir for 12 hours. Trifluoroacetic acid ( $\left.2.5 \mathrm{~mL}\right)$ was added at $78{ }^{\circ} \mathrm{C}$ and the solution was allowed to warm to $23^{\circ} \mathrm{C}$. Stirring continued for $1 \mathrm{~h}$. The reaction mixture was diluted with EtOAc $(60 \mathrm{~mL})$ and saturated $\mathrm{NaHCO}_{3}$ was added dropwise until gas evolution ceased. The mixture was added to a separatory funnel containing brine $(60 \mathrm{~mL})$. The aqueous layer was extracted with EtOAc $(3 \times 20 \mathrm{~mL})$. The organic layers were combined, dried over anhydrous $\mathrm{Na}_{2} \mathrm{SO}_{4}$ and concentrated. The residue was purified by flash column chromatography (50\% $\mathrm{Et}_{2} \mathrm{O} /$ hexanes) to afford $\beta$-hydroxy-dioxinone $9(1.25 \mathrm{~g}, 63 \%, 88 \%$ ee) as a colorless oil. Analytical data for 9: IR (film); 3462, 2953, 2857, 1728, 1635, 1386, 1255, 1205, 1091, 1012, $1091{ }^{1} \mathrm{H}$ NMR, $\left(500 \mathrm{MHz}, \mathrm{CDCl}_{3}\right) \delta 5.34$ (s, 1H), 4.15 (bs, 1H), 3.93-3.89 (m, 1H), $3.85-3.83(\mathrm{~m}, 1 \mathrm{H}), 2.43(\mathrm{dd}, J=14.2,7.81 \mathrm{~Hz}, 1 \mathrm{H}), 2.34(\mathrm{dd}, J=14.6,4.88 \mathrm{~Hz}, 1 \mathrm{H}), 1.8-1.65$

1. Pangborn, A. B.; Giardello, M. A.; Grubbs, R. H.; Rosen, R. K.; Timmers, F. J. Organomet. 1996, 15, 15181520.

2. Perrin, D. D. and Armarego, W. L. Purification of Laboratory Chemicals; 3rd Ed., Pergamon Press, Oxford. 1988. 
$(\mathrm{m}, 2 \mathrm{H}), 1.70(\mathrm{~s}, 3 \mathrm{H}), 1.69(\mathrm{~s}, 3 \mathrm{H}), 0.89(\mathrm{~s}, 9 \mathrm{H}), 0.08(\mathrm{~s}, 1 \mathrm{H}) ;{ }^{13} \mathrm{C} \mathrm{NMR}\left(125 \mathrm{MHz}, \mathrm{CDCl}_{3}\right) \delta$ 169.3, 161.4, 106.7, 95.3, 69.332, 62.6, 41.8, 38.0, 26.0, 25.5, 24.9, 18.3, -5.3; LRMS (ESI): Mass calcd for $\mathrm{C}_{16} \mathrm{H}_{30} \mathrm{O}_{5} \mathrm{SiNa}[\mathrm{M}+\mathrm{Na}]^{+}$, 353. Found $[\mathrm{M}+\mathrm{Na}]^{+}, 353 .[\alpha]_{\mathrm{D}}{ }^{25}=+17.5\left(\mathrm{CHCl}_{3}, \mathrm{c}=\right.$ 1.0, er $=94: 6$ ). Enantiomeric ratio was measured by HPLC (Chiralcel OD-H, 5\% IPA/Hexanes, $\left.\mathrm{Rt}_{1}=9.00, \mathrm{Rt}_{2}=10.17\right)$.

\section{HPLC data for Racemic 9}
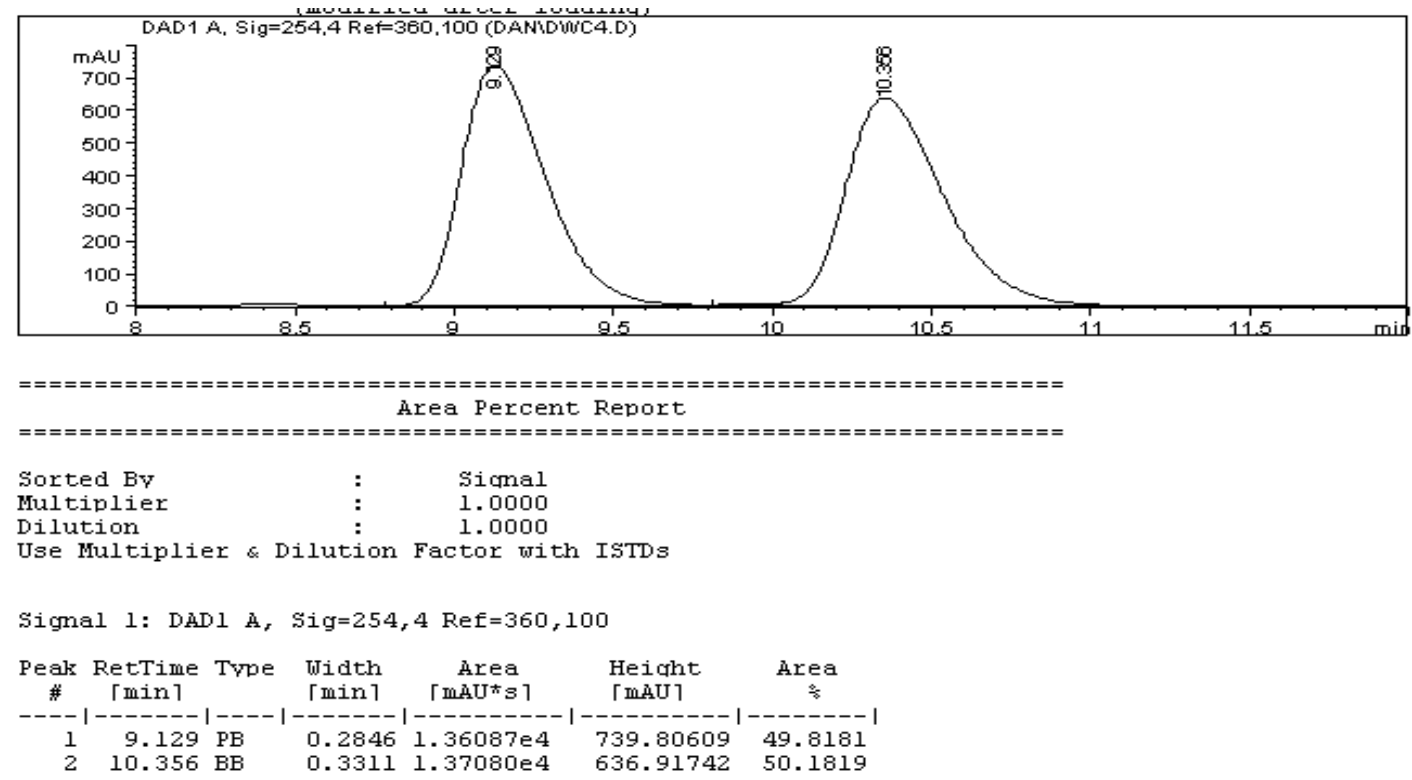

\section{HPLC data for Enantioenriched 9}
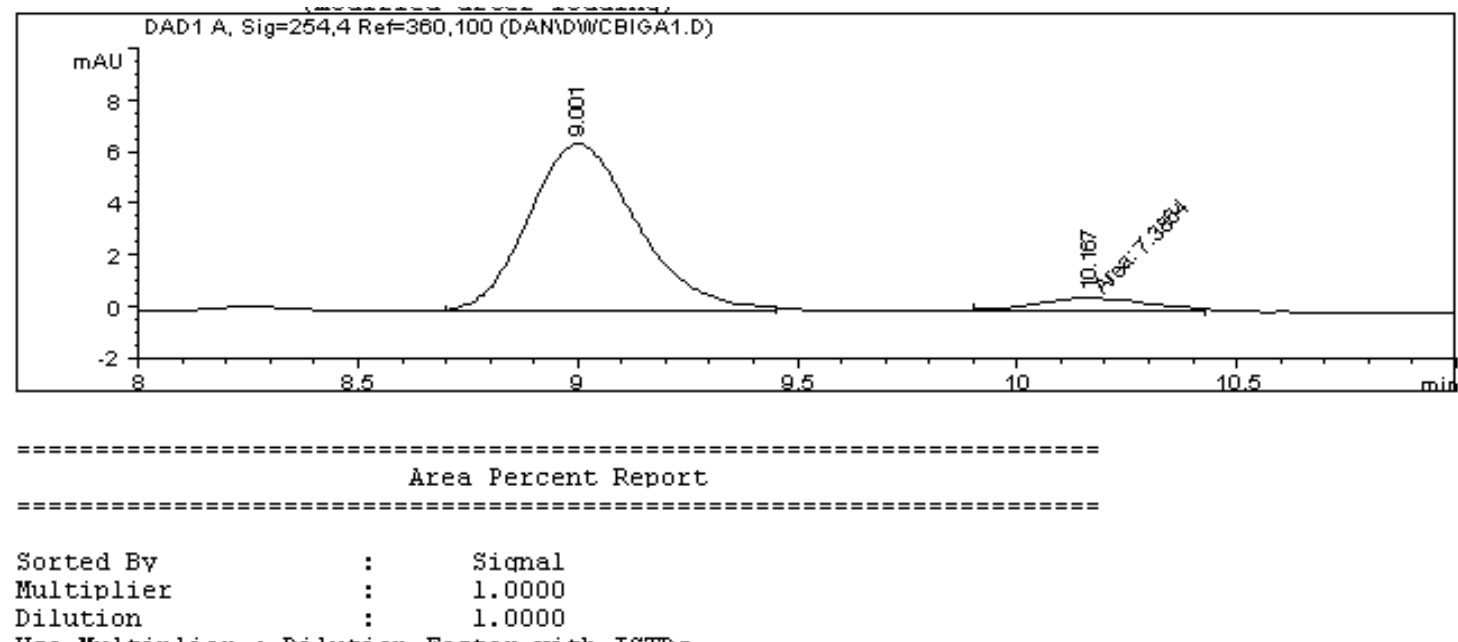

Use Multiplier \& Dilution Factor with ISTDs

Signal 1: DADl A, Sig $=254,4$ Ref $=360,100$

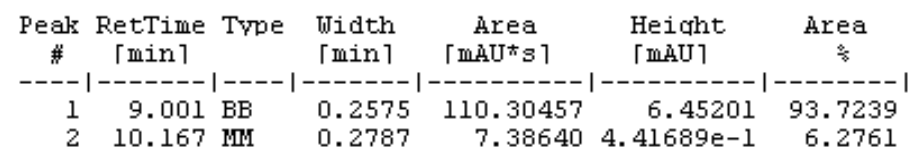




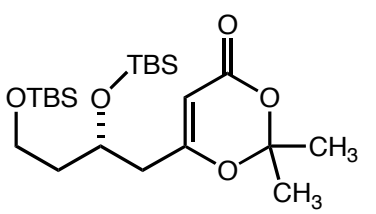

(S)-6-(2,4-bis(tert-butyldimethylsilyloxy)butyl)-2-hydroxybutyl)-2,2-dimethyl-4H-1,3-

dioxin-4-one (22): To a $0{ }^{\circ} \mathrm{C}$ solution of $9(1.63 \mathrm{~g}, 4.9 \mathrm{mmol})$ in $\mathrm{CH}_{2} \mathrm{Cl}_{2}(52 \mathrm{~mL})$ was added 2,6-lutidine $(1.7 \mathrm{~mL}, 14.8 \mathrm{mmol})$ and TBSOTf $(2.0 \mathrm{~mL}, 8.9 \mathrm{mmol})$. The resulting solution was stirred at $0{ }^{\circ} \mathrm{C}$ for $1 \mathrm{~h}$ and quenched by the addition of saturated $\mathrm{NaHCO}_{3}(80 \mathrm{~mL})$. The aqueous layer was extracted with EtOAc $(3 \times 15 \mathrm{~mL})$. The combined organic layers were dried over anhydrous $\mathrm{Na}_{2} \mathrm{SO}_{4}$, filtered and concentrated. The resulting residue was purified by flash column chromatography (40\% $\mathrm{Et}_{2} \mathrm{O} /$ hexanes) to afford silyl ether $22(1.98 \mathrm{~g}, 91 \%)$ as a clear oil. Analytical data: IR (film) 2955, 2932, 2858, 1734, 1636, 1386, 1254, 836, $776 \mathrm{~cm}^{-1}$; ${ }^{1} \mathrm{H}$ NMR $\left(500 \mathrm{MHz}, \mathrm{CDCl}_{3}\right) \delta 5.28(\mathrm{~s}, 1 \mathrm{H}), 4.15-4.13(\mathrm{~m}, 1 \mathrm{H}), 3.68(\mathrm{dd}, J=6.3,5.8 \mathrm{~Hz}, 2 \mathrm{H}), 2.40$ (ddd, $J$ $=20.0,14.1,5.86 \mathrm{~Hz}, 2 \mathrm{H}), 1.72-1.66(\mathrm{~m}, 2 \mathrm{H}), 1.70(\mathrm{~s}, 3 \mathrm{H}), 1.68(\mathrm{~s}, 3 \mathrm{H}), 1.57(\mathrm{~s}, 1 \mathrm{H}), 0.90(\mathrm{~s}$, 9H), $0.88(\mathrm{~s}, 9 \mathrm{H}), 0.08(\mathrm{~s}, 3 \mathrm{H}), 0.07(\mathrm{~s}, 3 \mathrm{H}), 0.06(\mathrm{~s}, 6 \mathrm{H}) ;{ }^{13} \mathrm{C}$ NMR $\left(125 \mathrm{MHz}, \mathrm{CDCl}_{3}\right) \delta 169.5$, 161.4, 106.5, 95.6, 66.8, 59.3, 42.4, 40.3, 26.1, 26.0, 25.9, 24.7, 18.4, 18.2, -4.3, -5.1; LRMS (ESI): Mass calcd for $\mathrm{C}_{22} \mathrm{H}_{44} \mathrm{O}_{5} \mathrm{Si}{ }_{2} \mathrm{Na}[\mathrm{M}+\mathrm{Na}]^{+}$, 468. Found $[\mathrm{M}+\mathrm{Na}], 468 .[\alpha]_{\mathrm{D}}^{25}=+3.1\left(\mathrm{CHCl}_{3}, \mathrm{c}\right.$ $=0.3$ ).

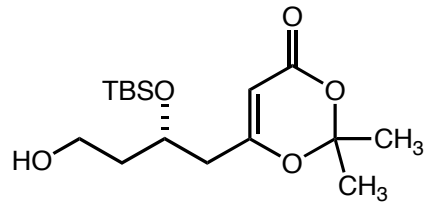

(S)-6-(2-(tert-butyldimethylsilyloxy)-4-hydroxybutyl)-2,2-dimethyl-4H-1,3-dioxin-4-one

(23): To a $23{ }^{\circ} \mathrm{C}$ solution of $22(1.82 \mathrm{~g}, 4.1 \mathrm{mmol})$ in EtOH $(178 \mathrm{~mL})$ was added PPTS $(2.48 \mathrm{~g}$, $9.8 \mathrm{mmol})$. The resulting mixture was stirred vigorously for $24 \mathrm{~h}$. Brine $(4 \mathrm{~mL})$ was added to the reaction mixture and the solvent was removed in vacuo and the resulting residue was diluted with EtOAc $(100 \mathrm{~mL})$ and added to a separatory funnel containing brine $(100 \mathrm{~mL})$. The aqueous layer was extracted with EtOAc $(4 \times 15 \mathrm{~mL})$. The organic layers were combined and dried with anhydrous $\mathrm{Na}_{2} \mathrm{SO}_{4}$, filtered and concentrated. The resulting residue was purified by flash column chromatography $\left(50 \% \mathrm{Et}_{2} \mathrm{O} /\right.$ hexanes $)$ to afford $\mathbf{2 3}(1.28 \mathrm{~g}, 83 \%)$ as a clear oil. Analytical data: IR (film) 3452, 2932, 2858, 1728, 1633, 1388, 1205, 1015, 835, $776 \mathrm{~cm}^{-1}$; ${ }^{1} \mathrm{H}$ NMR (500 MHz, $\left.\mathrm{CDCl}_{3}\right) \delta 5.29(\mathrm{~s}, 1 \mathrm{H}), 4.23-4.18(\mathrm{~m}, 1 \mathrm{H}), 3.83-3.73(\mathrm{~m}, 1 \mathrm{H}), 2.45$ (dddd, $J=22.9,20.0,14.1$, $5.85,2 \mathrm{H}), 1.98(\mathrm{bs}, 1 \mathrm{H}), 1.88-1.81(\mathrm{~m}, 1 \mathrm{H}), 1.75-1.65(\mathrm{~m}, 2 \mathrm{H}), 1.71(\mathrm{~s}, 3 \mathrm{H}), 1.69(\mathrm{~s}, 3 \mathrm{H}), 0.89$ (s, 9H), 0.11 (s, 3H), 0.09 (s, 3H); $\left.{ }^{13} \mathrm{C} \mathrm{NMR} \mathrm{(125} \mathrm{MHz,} \mathrm{CDCl}_{3}\right) \delta$ 168.9, 161.3, 128.6, 106.7, 95.7, 68.2, 59.6, 42.1, 38.9, 25.9, 24.7, 18.2, -4.5; LRMS (ESI): Mass calcd for $\mathrm{C}_{16} \mathrm{H}_{30} \mathrm{O}_{5} \mathrm{SiNa}$ $[\mathrm{M}+\mathrm{Na}]^{+}, 353$ Found $[\mathrm{M}+\mathrm{Na}] 353 .[\alpha]_{\mathrm{D}}{ }^{25}=-2.7\left(\mathrm{CHCl}_{3}, \mathrm{c}=1.0\right)$.<smiles>CC1(C)OC(=O)C=C(C[C@@H](CC(=O)O)O[GaH2])O1</smiles>

(R)-3-(tert-butyldimethylsilyloxy)-4-(2,2-dimethyl-4-oxo-4H-1,3-dioxin-6-yl)butanoic acid (5): To a $23{ }^{\circ} \mathrm{C}$ solution of $23(0.45 \mathrm{~g}, 1.36 \mathrm{mmol})$ in DMF $(21 \mathrm{~mL})$ was added PDC (2.61 g, 
$12.3 \mathrm{mmol})$. The resulting mixture was stirred vigorously for $3 \mathrm{~h}$. The reaction mixture was then diluted with $\mathrm{Et}_{2} \mathrm{O}(20 \mathrm{~mL})$ and then passed through a short plug of $\mathrm{MgSO}_{4}$ (eluting with $\left.\mathrm{Et}_{2} \mathrm{O}\right) . \quad \mathrm{H}_{2} \mathrm{O}(450 \mathrm{~mL})$ was then added to the filtrate and the aqueous layer was extracted with $\mathrm{Et}_{2} \mathrm{O}(5 \times 20 \mathrm{~mL})$. The combined organic layers were combined and dried with anhydrous $\mathrm{Na}_{2} \mathrm{SO}_{4}$, filtered and concentrated to afford carboxylic acid $\mathbf{5}(44 \mathrm{mg}, 97 \%)$ as a colorless oil. Carboxylic acid $\mathbf{5}$ was used directly in the next step. Analytical data: IR (film) 3101, 2931, 2857, 1734, 1635, 1388, 1205, 1092, 1015, 833, $777 \mathrm{~cm}^{-1}$; ${ }^{1} \mathrm{H}$ NMR $\left(500 \mathrm{MHz}, \mathrm{CDCl}_{3}\right) \delta 5.32$ (s, $1 \mathrm{H}), 4.42-4.40(\mathrm{~m}, 1 \mathrm{H}), 2.59(\mathrm{~d}, J=5.86,2 \mathrm{H}), 2.49(\mathrm{~d}, J=5.86 \mathrm{~Hz}, 2 \mathrm{H}), 1.72(\mathrm{~s}, 3 \mathrm{H}), 1.70(\mathrm{~s}$, $3 \mathrm{H}), 1.26(\mathrm{~s}, 1 \mathrm{H}), 0.88(\mathrm{~s}, 9 \mathrm{H}), 0.10(\mathrm{~s}, 3 \mathrm{H}), 0.09$ (s, 3H); ${ }^{13} \mathrm{C}$ NMR $(125 \mathrm{MHz}) \delta 176.3,168.2$, 161.1, 106.8, 96.1, 66.5, 42.2, 41.9, 29.9, 25.9, 25.8, 24.7, 18.1, -4.7; LRMS (ESI): Mass calcd for $\mathrm{C}_{16} \mathrm{H}_{28} \mathrm{O}_{6} \mathrm{SiNa}[\mathrm{M}+\mathrm{Na}]^{+}, 367$. Found $[\mathrm{M}+\mathrm{Na}] 367 .[\alpha]_{\mathrm{D}}^{25}=+3.3\left(\mathrm{CHCl}_{3}, \mathrm{c}=0.6\right)$.

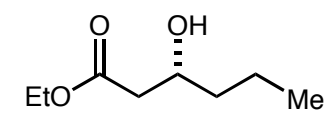

(R)-Ethyl 3-hydroxyhexanoate. A stainless steel stirred autoclave was charged with $\left[\mathrm{RuCl}_{2} \text { (benzene) }\right]_{2}(402 \mathrm{mg}, 0.80 \mathrm{mmol})$ and $(R)$-tol-BINAP $(1145 \mathrm{mg}, 1.69 \mathrm{mmol})$. The vessel was sealed and repeatedly pressure purged with argon (ca. $20 \times 30 \mathrm{psig}$ ). Argon sparged absolute ethanol $(950 \mathrm{~mL})$ and ethyl 3-oxohexanoate $(109.35 \mathrm{~g}, 682.6 \mathrm{mmol})$ were then added via cannula into the reaction vessel under argon. The vessel was sealed and pressure purged with argon and then hydrogen. The reactor was pressurized to and maintained at about 50 psig using a pressure regulator fed by a small high-pressure reservoir of hydrogen. The reaction mixture was vigorously stirred and heated to $100{ }^{\circ} \mathrm{C}$. Hydrogen uptake was complete within about $30 \mathrm{~min}$. of reaching $100^{\circ} \mathrm{C}$ after which heating continued for an additional $1 \mathrm{~h}$. After cooling and release of pressure, the orange product mixture was filtered and the filtrate was evaporated. Chiral GC (FID) analysis of the unpurified product mixture revealed 97\% ee. Vacuum distillation at about $125-130{ }^{\circ} \mathrm{C} / 90 \mathrm{mmHg}$ afforded $102.9 \mathrm{~g}(94 \%)$ of a clear, colorless oil. ${ }^{1} \mathrm{H}$ NMR $(400 \mathrm{MHz}$, $\left.\mathrm{CDCl}_{3}\right) \delta 4.16(\mathrm{q}, J=7.1 \mathrm{~Hz}, 2 \mathrm{H}) 4.01(\mathrm{~m}, 1 \mathrm{H}) 3.23(\mathrm{~s}, 1 \mathrm{H}) 2.48(\mathrm{dd}, J=16.2,3.6 \mathrm{~Hz}, 1 \mathrm{H}) 2.40$ $(\mathrm{dd}, J=16.2,8.6 \mathrm{~Hz}, 1 \mathrm{H}) 1.32-1.58(\mathrm{~m}, 4 \mathrm{H}) 1.27(\mathrm{dd}, J=7.1,7.1 \mathrm{~Hz}, 3 \mathrm{H}) 0.93(\mathrm{dd}, J=7.1,7.1$ $\mathrm{Hz}, 3 \mathrm{H}) .{ }^{13} \mathrm{C}$ NMR $\left(100 \mathrm{MHz}, \mathrm{CDCl}_{3}\right) \delta$ 172.2, 67.6, 60.4, 41.5, 38.7, 18.8, 14.3, 14.1 .

Chiral GC analysis was performed using an Agilent 6850 gas chromatograph equipped with an FID detector, Chiraldex $\beta$-cyclodextrin-DB ( $30 \mathrm{~m} \times 0.25 \mathrm{~mm}$ ) column, and a split injection port (50:1). Helium was used as the carrier gas at a constant $2 \mathrm{~mL} / \mathrm{min}$. The column oven was initially held at $60{ }^{\circ} \mathrm{C}$ for $1 \mathrm{~min}$ then ramped to $120^{\circ} \mathrm{C}$ at $2{ }^{\circ} \mathrm{C} / \mathrm{min}$. The chromatographic separation was checked using racemic ethyl 3-hydroxyhexanoate prepared analogously using racemic BINAP. The predominant isomer eluted second after about $25.2 \mathrm{~min}$ and was assigned to the $R$ configuration based upon literature precedent ${ }^{3}$ and Mosher ester analysis.

3. (a) Kitamura, M.; Tokunaga, M.; Ohkuma, T.; Noyori, R. Org. Synth. 1993, 71, 1. (b) Deng, L. S.; Huang, X. P.; Zhao, G. J. Org. Chem. 2006, 71, 4625. 


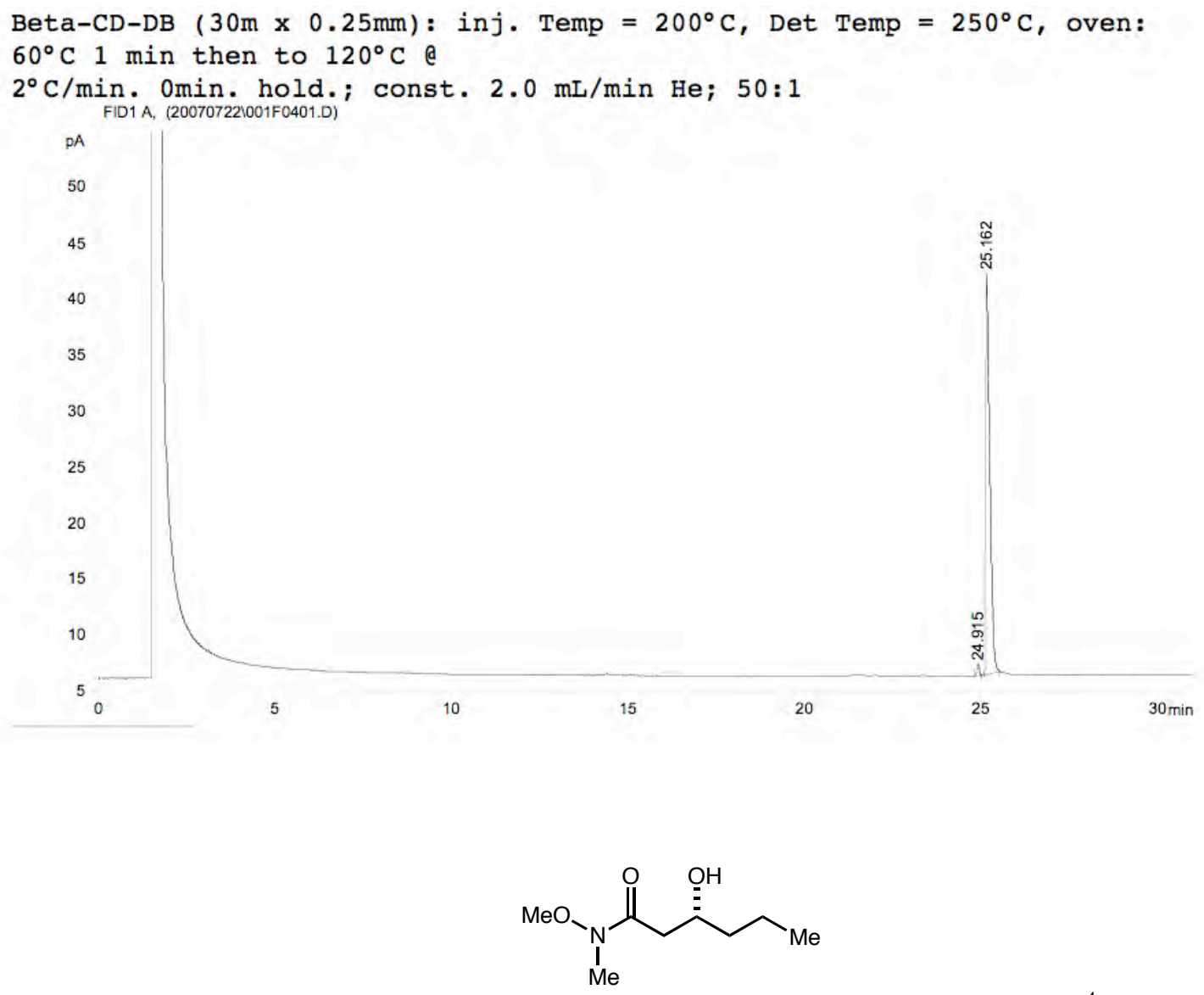

(R)-3-hydroxy- $N$-methoxy- $N$-methylhexanamide (24): Weinreb's amine ${ }^{4} \mathrm{MeONHMe} \cdot \mathrm{HCl}$ $(4.00 \mathrm{~g}, 41.0 \mathrm{mmol})$ was dried by azeotroping with benzene 3 times, dried in vacuo for $30 \mathrm{~min}$, and dissolved in THF $(20 \mathrm{~mL})$. (R)-Ethyl 3-hydroxyhexanoate $(2.12 \mathrm{~g}, 13.2 \mathrm{mmol})$ was then added and the mixture was cooled to $-20{ }^{\circ} \mathrm{C}$ (dry ice/benzyl alcohol). $i$ - $\mathrm{PrMgCl}(2.0 \mathrm{M}$ solution in THF, $39.7 \mathrm{~mL}$ ) was then added dropwise over $30 \mathrm{~min}$ via cannula, and the mixture was stirred for an additional $45 \mathrm{~min}$ at $-20^{\circ} \mathrm{C}$. The bath was then removed and the mixture stirred for $1 \mathrm{~h}$ at $23{ }^{\circ} \mathrm{C}$. The reaction was quenched with the dropwise addition of $20 \%$ aqueous $\mathrm{NH}_{4} \mathrm{Cl}(40 \mathrm{~mL})$. The aqueous layer was then extracted with EtOAc $(3 \times 40 \mathrm{~mL})$, the organic layers combined, dried over anhydrous $\mathrm{Na}_{2} \mathrm{SO}_{4}$, filtered and concentrated. The residue was purified by flash column chromatography (20\%-40\% gradient EtOAc/hexanes) to afford amide $\mathbf{2 4}(1.97 \mathrm{~g}, 85 \%)$ as a colorless oil. Analytical data for 24: IR (film) 3448, 2958, 2936, 2874, 1647, 1420, 1388, 1122, 1178, $1000 \mathrm{~cm}^{-1} ;{ }^{1} \mathrm{H}$ NMR $\left(500 \mathrm{MHz}, \mathrm{CDCl}_{3}\right) \delta 4.00(\mathrm{~m}, 1 \mathrm{H}), 3.78(\mathrm{~s}, 1 \mathrm{H}), 3.66(\mathrm{~s}, 3 \mathrm{H})$, $3.16(\mathrm{~s}, 3 \mathrm{H}), 2.63(\mathrm{~d}, J=16.5 \mathrm{~Hz}, 1 \mathrm{H}), 2.42(\mathrm{dd}, J=17.0,9.5 \mathrm{~Hz}, 1 \mathrm{H}), 1.56-1.44(\mathrm{~m}, 2 \mathrm{H}), 1.38$ (dddd, $J=22.0,15.5,15.5,4.0 \mathrm{~Hz}, 2 \mathrm{H}), 0.90(\mathrm{dd}, J=7.5,7.5 \mathrm{~Hz}, 3 \mathrm{H}) ;{ }^{13} \mathrm{C}$ NMR $(125 \mathrm{MHz}$, $\left.\mathrm{CDCl}_{3}\right) \delta 174.1,67.7,61.4,38.8,38.3,31.9,18.9,14.2$; LRMS (ESI): Mass calcd for $\mathrm{C}_{16} \mathrm{H}_{34} \mathrm{~N}_{2} \mathrm{O}_{6} \mathrm{Na}[2 \mathrm{M}+\mathrm{Na}]^{+}, 373$. Found [2M+Na], $373 ;[\alpha]_{\mathrm{D}}^{25}=-42.4\left(\mathrm{CHCl}_{3}, \mathrm{c}=0.79\right)$.

4. Nahm, S.; Weinreb, S. M. Tetrahedron Lett. 1981, 22, 3815-3818. 


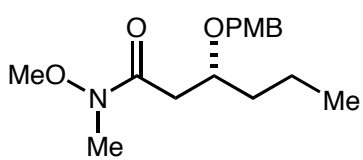

(R)-3-(4-methoxybenzyloxy)- $\boldsymbol{N}$-methoxy- $\boldsymbol{N}$-methylhexanamide (11): Amide 24 (1.40 g, 8.00 mmol) was dissolved in cyclohexane $(7 \mathrm{~mL})$ and $\mathrm{CH}_{2} \mathrm{Cl}_{2}(3 \mathrm{~mL})$ and cooled to $0{ }^{\circ} \mathrm{C}$. Freshly prepared PMB-imidate $(2.71 \mathrm{~g}, 9.60 \mathrm{mmol})$ was dissolved in $\mathrm{CH}_{2} \mathrm{Cl}_{2}(2 \mathrm{~mL})$ and added dropwise, followed by the addition of pPTs $(0.10 \mathrm{~g}, 0.40 \mathrm{mmol})$ in one portion. The mixture was then warmed to $23{ }^{\circ} \mathrm{C}$ and stirred for $8 \mathrm{~h}$ after which an additional portion of pPTs was added $(0.10 \mathrm{~g}, 0.40 \mathrm{mmol})$. This mixture was stirred for an additional $7 \mathrm{~h}$, and then plug filtered through a pad of silica ( $20 \% \mathrm{EtOAc/hexanes),} \mathrm{and} \mathrm{the} \mathrm{filtrate} \mathrm{washed} \mathrm{with} \mathrm{saturated} \mathrm{aqueous}$ $\mathrm{NaHCO}_{3}$ and brine then dried over anhydrous $\mathrm{Na}_{2} \mathrm{SO}_{4}$, filtered and concentrated. The residue was purified by flash column chromatography (15\%-30\% gradient EtOAc/hexanes) to afford amide 11 (1.87 g, 80\%) as a yellow oil. Analytical data for 11: IR (film) 3287, 2960, 2872, 1732, 1661, 1614, 1515, 1462, 1385, 1249, 1175, $1036 \mathrm{~cm}^{-1} ;{ }^{1} \mathrm{H}$ NMR $\left(500 \mathrm{MHz}, \mathrm{CDCl}_{3}\right) \delta 7.20$ $(\mathrm{d}, J=8.5 \mathrm{~Hz}, 2 \mathrm{H}), 6.80(\mathrm{~d}, J=7.5 \mathrm{~Hz}, 2 \mathrm{H}), 4.45\left(\mathrm{~A}\right.$ of $\left.\mathrm{ABq}, J_{\mathrm{AB}}=10.5 \mathrm{~Hz}, 1 \mathrm{H}\right), 4.41(\mathrm{~B}$ of $\left.\mathrm{ABq}, J_{\mathrm{AB}}=10.5 \mathrm{~Hz}, 1 \mathrm{H}\right), 3.92(\mathrm{ddd}, J=11.5,5.5,5.5 \mathrm{~Hz}, 1 \mathrm{H}), 3.72(\mathrm{~s}, 3 \mathrm{H}), 3.60(\mathrm{~s}, 3 \mathrm{H}), 3.14$ (s, 3H), 2.80 (dd, $J=14.5,6.5 \mathrm{~Hz}, 1 \mathrm{H}), 2.41$ (dd, $J=15.5,4.5 \mathrm{~Hz}, 1 \mathrm{H}), 1.58-1.39$ (m, $3 \mathrm{H}), 1.33$ (dddd, $J=23.5,13.5,13.5,6.5 \mathrm{~Hz}, 1 \mathrm{H}), 0.87(\mathrm{dd}, J=7.5,7.5 \mathrm{~Hz}, 3 \mathrm{H}) ;{ }^{13} \mathrm{C}$ NMR $(125 \mathrm{MHz}$, $\left.\mathrm{CDCl}_{3}\right) \delta 172.6,159.0,130.8,129.3,113.6,75.7,71.5,61.2,55.1,37.2,37.1,32.0,18.6,14.1$; LRMS (ESI): Mass calcd for $\mathrm{C}_{32} \mathrm{H}_{50} \mathrm{~N}_{2} \mathrm{O}_{8} \mathrm{Na}[2 \mathrm{M}+\mathrm{Na}]^{+}, 614$. Found $[2 \mathrm{M}+\mathrm{Na}], 613 ;[\alpha]_{\mathrm{D}}^{25}=$ $+8.5\left(\mathrm{CHCl}_{3}, \mathrm{c}=1.0\right)$.

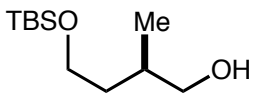

(R)-4-(tert-butyldimethylsilyloxy)-2-methylbutan-1-ol (25): Diisopropylamine (23.8 g, 32.9 $\mathrm{mL}, 234 \mathrm{mmol})$, was dissolved in THF $(200 \mathrm{~mL})$ and cooled to $-78{ }^{\circ} \mathrm{C}$. A solution of $n$ butyllithium (1.59 $\mathrm{M}$ in hexanes, $137 \mathrm{~mL}$ ) was added dropwise by syringe and the solution was stirred for $10 \mathrm{~min}$ at $-78{ }^{\circ} \mathrm{C}$, then warmed to $0{ }^{\circ} \mathrm{C}$ and stirred for an additional $15 \mathrm{~min}$. Ammonia-borane complex $(6.90 \mathrm{~g}, 224 \mathrm{mmol})$ was then added in one portion and the mixture stirred for $15 \mathrm{~min}$ at $0{ }^{\circ} \mathrm{C}$, then warmed to $23{ }^{\circ} \mathrm{C}$ and stirred for an additional $15 \mathrm{~min}$. The mixture was then cooled back down to $0{ }^{\circ} \mathrm{C}$ and $[1 S(R), 2 S]-N$-(2-Hydroxy-1-methyl-2phenylethyl)-4-(tert-butyldimethylsilyloxy)- $N, 2-$ dimethylbutanamide $^{5} \quad(21.2 \mathrm{~g}, \quad 55.9 \mathrm{mmol})$ dissolved in THF $(120 \mathrm{~mL})$ was added via cannula, and the mixture as warmed to $23{ }^{\circ} \mathrm{C}$ and stirred for $2 \mathrm{~h}$. The mixture was then cooled to $0{ }^{\circ} \mathrm{C}$ and quenched with the addition of $0.01 \mathrm{M}$ $\mathrm{HCl}(500 \mathrm{~mL})$. This mixture was then extracted with EtOAc $(3 \times 200 \mathrm{~mL})$ and the organic layers were combined, washed with $0.1 \mathrm{M} \mathrm{HCl}(100 \mathrm{~mL}), 1 \mathrm{M} \mathrm{NaOH}(100 \mathrm{~mL})$, brine $(100 \mathrm{ML})$ then dried over anhydrous $\mathrm{Na}_{2} \mathrm{SO}_{4}$, filtered and concentrated. The residue was purified by flash column chromatography (10\% EtOAc/hexanes) to afford alcohol 25 (11.3 g, 90\%) as a colorless oil. Analytical data for 25: IR (film) 3352, 2930, 2859, 1464, 1389, 1253, 1095, 1085, 1044, 1001, 835, $776 \mathrm{~cm}^{-1} ;{ }^{1} \mathrm{H}$ NMR (500 MHz, $\mathrm{CDCl}_{3}$ ) $\delta 3.72$ (ddd, $\left.J=10.5,6.0,6.0 \mathrm{~Hz}, 1 \mathrm{H}\right), 3.62$ (ddd, $J=7.5,7.5,4.0 \mathrm{~Hz}, 1 \mathrm{H}), 3.45$ (ddd, $J=16.5,5.5,5.5 \mathrm{~Hz}, 1 \mathrm{H}), 3.37$ (ddd, $J=15.0,4.0,4.0$ $\mathrm{Hz}, 1 \mathrm{H}), 3.29$ (s $1 \mathrm{H}), 1.75$ (ddd, $J=12.5,12.5,6.5,1 \mathrm{H}), 1.56-1.44(\mathrm{~m}, 2 \mathrm{H}), 0.88(\mathrm{~d}, J=7.0 \mathrm{~Hz}$,

5. Myers, A. G.; McKinstry, L. J. Org. Chem. 1996, 61, 2428-2440. 
3H), 0.86 (s, 9H), 0.03 (s, 6H); ${ }^{13} \mathrm{C}$ NMR (125 MHz, $\left.\mathrm{CDCl}_{3}\right) \delta$ 68.2, 61.9, 37.6, 34.4, 26.0, 18.4, 17.5, -5.3; LRMS (ESI): Mass calcd for $\mathrm{C}_{11} \mathrm{H}_{26} \mathrm{O}_{2} \mathrm{Si}[\mathrm{M}]^{+}$, 218. Found [M], 218; $[\alpha]_{\mathrm{D}}{ }^{25}=+9.8$ $\left(\mathrm{CHCl}_{3}, \mathrm{c}=1.0\right)$.

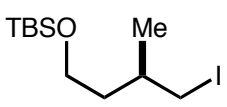

((R)-4-iodo-3-methylbutoxy)(tert-butyl)dimethylsilane (12): Triphenylphosphine $(8.21 \mathrm{~g}, 31.3$ mmol) was dissolved in $\mathrm{CH}_{2} \mathrm{Cl}_{2}(130 \mathrm{~mL}$ and imidazole $(2.66 \mathrm{~g}, 39.2 \mathrm{mmol})$ was added in one portion followed by $\mathrm{I}_{2}(9.27 \mathrm{~g}, 36.5 \mathrm{mmol})$. A solution of alcohol 25 in $\mathrm{CH}_{2} \mathrm{Cl}_{2}(50 \mathrm{~mL})$ was then added via cannula and the mixture stirred for $15 \mathrm{~min}$ at $23{ }^{\circ} \mathrm{C}$. The solvent was removed in vacuo and the residue was purified by flash column chromatography ( $5 \% \mathrm{Et}_{2} \mathrm{O} / \mathrm{hexanes}$ ) to afford iodide $12(6.02 \mathrm{~g}, 70 \%)$ as a tan oil. Analytical data for 12: IR (film) 3746, 2929, 2857 , 2362, 1652, 1464, 1253, 1099, 834, $775 \mathrm{~cm}^{-1} ;{ }^{1} \mathrm{H}$ NMR $\left(500 \mathrm{MHz}, \mathrm{CDCl}_{3}\right) \delta 3.63(\mathrm{dddd}, J=$ $16.5,10.5,10.5,6.0 \mathrm{~Hz}, 2 \mathrm{H}), 3.27$ (dd, $J=9.5,4.5 \mathrm{~Hz}, 1 \mathrm{H}), 3.19$ (dd, $J=10.0,6.0 \mathrm{~Hz}, 1 \mathrm{H})$, $1.67-1.56(\mathrm{~m}, 2 \mathrm{H}), 1.42(\mathrm{ddd}, J=13.0,13.0,6.5 \mathrm{~Hz}, 1 \mathrm{H}), 0.98(\mathrm{~d}, J=6.5 \mathrm{~Hz}, 3 \mathrm{H}), 0.88(\mathrm{~s}, 9 \mathrm{H})$, $0.04(\mathrm{~s}, 6 \mathrm{H}) ;{ }^{13} \mathrm{C}$ NMR $\left(125 \mathrm{MHz}, \mathrm{CDCl}_{3}\right) \delta 60.8,39.9,31.5,26.1,20.8,18.4,18.4,-5.1 ;[\alpha]_{\mathrm{D}}{ }^{25}$ $=-4.9\left(\mathrm{CHCl}_{3}, \mathrm{c}=1.0\right)$.

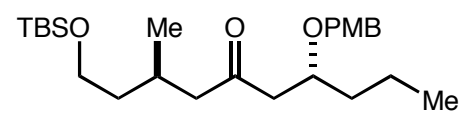

(3R,7R)-1-(tert-butyldimethylsilyloxy)-7-(4-methoxybenzyloxy)-3-methyldecan-5-one (13): Iodide 12 ( $2.04 \mathrm{~g}, 6.20 \mathrm{mmol})$ was dried by azeotroping with benzene 3 times then dissolved in pentane (freshly distilled from $\mathrm{CaH}_{2}, 35 \mathrm{~mL}$ ) and ether $(25 \mathrm{~mL})$ and cooled to $-78{ }^{\circ} \mathrm{C}$. A solution of $t$-butyllithium $(1.7 \mathrm{M}$ in pentane, $7.30 \mathrm{~mL})$ was added dropwise and the mixture was stirred at $-78{ }^{\circ} \mathrm{C}$ for $15 \mathrm{~min}$, then warmed to $0{ }^{\circ} \mathrm{C}$ for $20 \mathrm{~min}$ (after which a white precipitate was generated). The solution was cooled back down to $-78{ }^{\circ} \mathrm{C}$ and a solution of amide 11 (1.02 g, $3.45 \mathrm{mmol}$ ) in THF was cooled to $-78{ }^{\circ} \mathrm{C}$ and added via cannula (dripped along the side of the cooled reaction flask). This mixture was stirred for $15 \mathrm{~min}$ at $-78{ }^{\circ} \mathrm{C}$. The reaction was quenched at low temperature with the addition of saturated aqueous $\mathrm{NH}_{4} \mathrm{Cl}(50 \mathrm{~mL})$. Upon warming to ambient temperature, the mixture was extracted with EtOAc $(3 \times 50 \mathrm{~mL})$, the organic layers combined, dried over anhydrous $\mathrm{Na}_{2} \mathrm{SO}_{4}$, filtered and concentrated. The residue was purified by flash column chromatography (3\%-10\% gradient EtOAc/hexanes) to afford ketone $13(0.74 \mathrm{~g}, 50 \%)$ as a colorless oil. Analytical data for 13: IR (film) 2957, 2860, 2362, 1713, 1614, 1514, 1465, 1362, 1302, 1250, 1094, 1039, $835 \mathrm{~cm}^{-1} ;{ }^{1} \mathrm{H}$ NMR (500 MHz, $\left.\mathrm{CDCl}_{3}\right) \delta 7.22$ $(\mathrm{d}, J=8.0 \mathrm{~Hz}, 2 \mathrm{H}), 6.84(\mathrm{~d}, J=8.5 \mathrm{~Hz}, 2 \mathrm{H}), 4.42$ (s. $2 \mathrm{H}), 3.92(\mathrm{ddd}, J=11.5,6.0,6.0 \mathrm{~Hz}, 1 \mathrm{H})$, 3.77 (s, 3H), 3.62 (dddd, $J=7.5,7.5,7.5,6.0 \mathrm{~Hz}, 2 \mathrm{H}), 2.71$ (dd, $J=16.5,7.5 \mathrm{~Hz}, 1 \mathrm{H}), 2.43$ (dddd, $J=25.0,9.0,9.0,9.0 \mathrm{~Hz}, 2 \mathrm{H}), 2.24(\mathrm{dd}, J=16.0,8.0 \mathrm{~Hz}, 1 \mathrm{H}), 2.15$ (ddd, $J=13.5,13.5$, $6.5 \mathrm{~Hz}, 1 \mathrm{H}), 1.55-1.44(\mathrm{~m}, 4 \mathrm{H}), 1.43-1.33(\mathrm{~m}, 2 \mathrm{H}), 0.95-0.86(\mathrm{~m}, 6 \mathrm{H}), 0.88(\mathrm{~s}, 9 \mathrm{H}), 0.03(\mathrm{~s}, 6 \mathrm{H})$; ${ }^{13} \mathrm{C}$ NMR $\left(125 \mathrm{MHz}, \mathrm{CDCl}_{3}\right) \delta 209.8,159.4,131.0,129.6,113.9,75.3,71.5,61.3,55.5,51.8$, 48.3, 39.8, 37.0, 26.4, 26.2, 20.1, 18.8, 18.5, 14.4, -5.1; LRMS (ESI): Mass calcd for $\mathrm{C}_{50} \mathrm{H}_{88} \mathrm{O}_{8} \mathrm{Si}_{2} \mathrm{Na}[2 \mathrm{M}+\mathrm{Na}]^{+}, 896$. Found $[2 \mathrm{M}+\mathrm{Na}], 897 ;[\alpha]_{\mathrm{D}}{ }^{25}=-5.0\left(\mathrm{CHCl}_{3}, \mathrm{c}=1.0\right)$. 


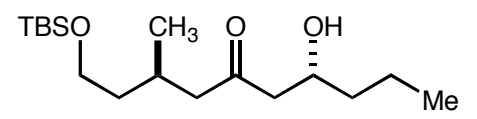

(3R,7R)-1-(tert-butyldimethylsilyloxy)-7-hydroxy-3-methyldecan-5-one (26): Ketone 13 (0.52 $\mathrm{g}, 1.2 \mathrm{mmol})$ was dissolved in $\mathrm{CH}_{2} \mathrm{Cl}_{2}(110 \mathrm{~mL})$, $\mathrm{pH} 7$ phosphate buffer added $(10 \mathrm{~mL})$ and the mixture cooled to $0{ }^{\circ} \mathrm{C}$. DDQ added $(0.32 \mathrm{~g}, 1.4 \mathrm{mmol})$ and the mixture stirred for $3 \mathrm{~h}$ at $0{ }^{\circ} \mathrm{C}$. The reaction was quenched by the addition of saturated aqueous $\mathrm{NaHCO}_{3}(50 \mathrm{~mL})$, and the aqueous phase was extracted with $\mathrm{CH}_{2} \mathrm{Cl}_{2}(3 \times 50 \mathrm{~mL})$. The organic layers were combined, dried over anhydrous $\mathrm{Na}_{2} \mathrm{SO}_{4}$, filtered and concentrated. The residue was purified by flash column chromatography (3\%-5\% gradient EtOAc/hexanes) to afford ketone $26(0.32 \mathrm{~g}, 84 \%)$ as a colorless oil. Analytical data for 26: IR (film) 3463, 2957, 2860, 1701, 1465, 1254, 1096, 837, $776 \mathrm{~cm}^{-1} ;{ }^{1} \mathrm{H}$ NMR $\left(500 \mathrm{MHz}, \mathrm{CDCl}_{3}\right) \delta 4.02(\mathrm{~m}, 1 \mathrm{H}), 3.62$ (dddd, $J=10.5,10.5,10.5,6.5 \mathrm{~Hz}$, $2 \mathrm{H}), 3.07(\mathrm{~d}, J=3.5 \mathrm{~Hz}, 1 \mathrm{H}), 2.55(\mathrm{dd}, J=17.5,2.0 \mathrm{~Hz}, 1 \mathrm{H}), 2.50-2.43(\mathrm{~m}, 2 \mathrm{H}), 2.24(\mathrm{dd}, J=$ 16.0, $8.5 \mathrm{~Hz}, 1 \mathrm{H}), 2.15$ (dddd, $J=13.0,13.0,13.0,6.0,1 \mathrm{H}), 1.52-1.43$ (m, 3H), 1.41-1.29 (m, $3 \mathrm{H}), 0.92-0.87(\mathrm{~m}, 6 \mathrm{H}), 0.87(\mathrm{~s}, 9 \mathrm{H}), 0.03(\mathrm{~s}, 6 \mathrm{H}) ;{ }^{13} \mathrm{C} \mathrm{NMR}\left(125 \mathrm{MHz}, \mathrm{CDCl}_{3}\right) \delta 212.4,67.4$, 61.1, 51.3, 49.5, 39.7, 38.7, 26.3, 26.1, 20.1, 18.8, 18.4, 14.2, -5.1; LRMS (ESI): Mass calcd for $\mathrm{C}_{34} \mathrm{H}_{72} \mathrm{O}_{6} \mathrm{Si}_{2} \mathrm{Na}[2 \mathrm{M}+\mathrm{Na}]^{+}, 656$. Found $[2 \mathrm{M}+\mathrm{Na}], 655 ;[\alpha]_{\mathrm{D}}^{25}=+23.4\left(\mathrm{CHCl}_{3}, \mathrm{c}=0.82\right)$.

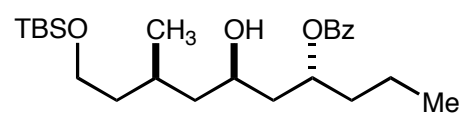

(4R,6R,8R)-10-(tert-butyldimethylsilyloxy)-6-hydroxy-8-methyldecan-4-yl benzoate (14): Ketone $26(0.32 \mathrm{~g}, 1.0 \mathrm{mmol})$ and benzaldehyde $(0.53 \mathrm{~g}, 0.51 \mathrm{~mL}, 5.0 \mathrm{mmol})$ were dissolved in THF (4 mL) that was freshly distilled over benzophenone and sodium, then cooled to $-10{ }^{\circ} \mathrm{C}$ (ice/salt water). A freshly prepared $0.1 \mathrm{M}$ solution of $\operatorname{SmI}_{2}(10.1 \mathrm{~mL})^{6}$ was added dropwise and the mixture stirred at $-10{ }^{\circ} \mathrm{C}$ for $12 \mathrm{~h}$. The reaction was quenched at low temperature by the addition of saturated aqueous $\mathrm{NaHCO}_{3}(5 \mathrm{~mL})$, and the aqueous layer was extracted with $\mathrm{Et}_{2} \mathrm{O}(3$ x $20 \mathrm{~mL}$ ). The organic layers were combined, dried over anhydrous $\mathrm{Na}_{2} \mathrm{SO}_{4}$, filtered and concentrated. The residue was purified by flash column chromatography $(5 \%-7.5 \%$ gradient EtOAc/hexanes) to afford alcohol $14(0.39 \mathrm{~g}, 91 \%)$ as a colorless oil. Analytical data for 14: IR (film) 3519, 2957, 2930, 2858, 2362, 1700, 1459, 1277, 1097, $837 \mathrm{~cm}^{-1}$; ${ }^{1} \mathrm{H}$ NMR (500 MHz, $\left.\mathrm{CDCl}_{3}\right) \delta 8.05(\mathrm{~d}, J=7.5 \mathrm{~Hz}, 2 \mathrm{H}), 7.57(\mathrm{dd}, J=7.5,7.5 \mathrm{~Hz}, 1 \mathrm{H}), 7.45(\mathrm{~d}, J=8.0 \mathrm{~Hz}, 2 \mathrm{H}), 5.36$ $(\mathrm{m}, 1 \mathrm{H}), 3.66-3.55(\mathrm{~m}, 3 \mathrm{H}), 3.26(\mathrm{~d}, J=3.5 \mathrm{~Hz}, 1 \mathrm{H}), 1.80-1.71(\mathrm{~m}, 3 \mathrm{H}), 1.66-1.50(\mathrm{~m}, 3 \mathrm{H})$, 1.47-1.24 (m, 5H), $0.93(\mathrm{t}, J=7.5 \mathrm{~Hz}, 3 \mathrm{H}), 0.86(\mathrm{~d}, J=6.5 \mathrm{~Hz}, 3 \mathrm{H}), 0.85(\mathrm{~s}, 9 \mathrm{H}),-0.01(\mathrm{~s}, 6 \mathrm{H})$; ${ }^{13} \mathrm{C}$ NMR $\left(125 \mathrm{MHz}, \mathrm{CDCl}_{3}\right) \delta$ 167.9, 133.4, 130.3, 128.7, 72.5, 65.4, 61.6, 44.7, 43.5, 39.7, 37.5, 26.6, 26.2, 20.7, 19.0, 18.5, 14.2, -5.0; LRMS (ESI): Mass calcd for $\mathrm{C}_{48} \mathrm{H}_{84} \mathrm{O}_{8} \mathrm{Si}_{2} \mathrm{Na}[2 \mathrm{M}+$ $\mathrm{Na}]^{+}, 868$. Found $[2 \mathrm{M}+\mathrm{Na}], 867 ;[\alpha]_{\mathrm{D}}^{25}=+4.1\left(\mathrm{CHCl}_{3}, \mathrm{c}=0.70\right)$.

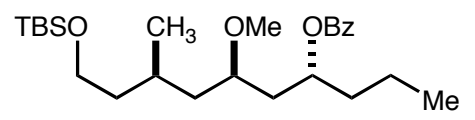

(4R,6R,8R)-10-(tert-butyldimethylsilyloxy)-6-methoxy-8-methyldecan-4-yl benzoate (27): Alcohol 14 (0.098 g, $0.23 \mathrm{mmol})$ was dissolved in $\mathrm{CH}_{2} \mathrm{Cl}_{2}(1 \mathrm{~mL})$ and 2,6-di-tert-butyl-4methylpyridne $(0.238 \mathrm{~g}, 1.16 \mathrm{mmol})$ was added. This solution was cooled to $0{ }^{\circ} \mathrm{C}$ and freshly

6. Girard, P.; Namy, J. L.; Kagan, H. B. J. Am. Chem. Soc. 1980, 102, 2693-2698. 
prepared MeOTf $(0.190 \mathrm{~g}, 0.13 \mathrm{~mL}, 1.16 \mathrm{mmol})$ added dropwise. The ice bath was then removed and the mixture stirred for $12 \mathrm{~h}$ at $23{ }^{\circ} \mathrm{C}$ (formation of a white precipitate observed). After $12 \mathrm{~h}$, a second portion of MeOTf $(0.190 \mathrm{~g}, 0.13 \mathrm{~mL}, 1.16 \mathrm{mmol})$ was added and the mixture was allowed to stir for an additional $8 \mathrm{~h}$. The mixture was then concentrated in vacuo and the residue was purified by flash column chromatography (3\%-5\% gradient EtOAc/hexanes) to afford methylated alcohol $27(0.088 \mathrm{~g}, 88 \%)$ as a colorless oil. Analytical data for 27: IR (film) 2931, 2858, 2362, 1273, 1096, 836, $776 \mathrm{~cm}^{-1} ;{ }^{1} \mathrm{H}$ NMR $\left(500 \mathrm{MHz}, \mathrm{CDCl}_{3}\right) \delta 8.04(\mathrm{~d}, J=$ $7.5 \mathrm{~Hz}, 2 \mathrm{H}), 7.55(\mathrm{dd}, J=7.5,7.5 \mathrm{~Hz}, 1 \mathrm{H}), 7.44(\mathrm{dd}, J=7.5,7.5 \mathrm{~Hz}, 2 \mathrm{H}), 5.38$ (ddd, $J=12.0$, 9.0, 3.0 Hz, 1H), 3.63 (dddd, $J=17.0,10.5,10.5,6.5 \mathrm{~Hz}, 2 \mathrm{H}), 3.28(\mathrm{~m}, 3 \mathrm{H}), 3.24(\mathrm{~m}, 1 \mathrm{H}), 1.85$ $(\mathrm{m}, 1 \mathrm{H}), 1.74-1.60(\mathrm{~m}, 4 \mathrm{H}), 1.55(\mathrm{ddd}, J=13.0,7.0,7.0,1 \mathrm{H}), 1.48-1.30(\mathrm{~m}, 5 \mathrm{H}) 0.92(\mathrm{t}, J=7.0$ $\mathrm{Hz}, 3 \mathrm{H}), 0.88(\mathrm{~s}, 9 \mathrm{H}), 0.85(\mathrm{~d}, J=7.0 \mathrm{~Hz}, 3 \mathrm{H}), 0.03(\mathrm{~s}, 6 \mathrm{H}) ;{ }^{13} \mathrm{C}$ NMR $\left(125 \mathrm{MHz}, \mathrm{CDCl}_{3}\right) \delta$ $166.4,132.9,130.9,129.7,128.5,76.3,72.3,61.2,57.2,41.6,40.5,39.9,37.5,26.3,26.2,20.0$, 18.7, 18.5, 14.3, -5.0; LRMS (ESI): Mass calcd for $\mathrm{C}_{25} \mathrm{H}_{44} \mathrm{O}_{4} \mathrm{Si}[\mathrm{M}]^{+}$, 437. Found [M], 437; $[\alpha]_{\mathrm{D}}^{25}=-14.5\left(\mathrm{CHCl}_{3}, \mathrm{c}=0.75\right)$.

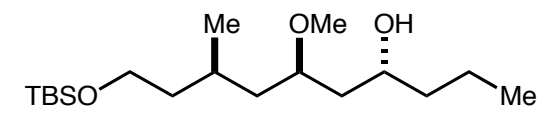

(4R,6R,8R)-10-(tert-butyldimethylsilyloxy)-6-methoxy-8-methyldecan-4-ol (6): Methylated alcohol 27 (0.078 g, $0.180 \mathrm{mmol})$ was dissolved in $\mathrm{MeOH}(2 \mathrm{~mL})$ and $\mathrm{K}_{2} \mathrm{CO}_{3}(1.24 \mathrm{~g}, 8.98$ mmol) added. This slurry was stirred at $23{ }^{\circ} \mathrm{C}$ for $20 \mathrm{~h}$. The mixture was then concentrated in vacuo and the residue was diluted with water, and extracted with $\mathrm{Et}_{2} \mathrm{O}(3 \times 15 \mathrm{~mL})$. The organic layers were combined, dried over anhydrous $\mathrm{Na}_{2} \mathrm{SO}_{4}$, filtered and concentrated. The residue was purified by flash column chromatography (5\%-10\% gradient EtOAc/hexanes) to afford alcohol 6 $(0.052 \mathrm{~g}, 86 \%)$ as a colorless oil. Analytical data for 6: IR (film) 3454, 2955, 2932, 1464, 1253, 1094, $836 \mathrm{~cm}^{-1} ;{ }^{1} \mathrm{H}$ NMR (500 MHz, $\left.\mathrm{CDCl}_{3}\right) \delta 3.89(\mathrm{~m}, 1 \mathrm{H}), 3.64$ (ddd, $J=24.5,10.0,6.0 \mathrm{~Hz}$, 2H), 3.56 (ddd, $J=10.0,10.0,6.5 \mathrm{~Hz}, 1 \mathrm{H}), 3.36$ (s, 3H), $3.06(\mathrm{~d}, J=3.0 \mathrm{~Hz}, 1 \mathrm{H}), 1.72(\mathrm{~m}, 1 \mathrm{H})$, $1.65-1.31(\mathrm{~m}, 10 \mathrm{H}), 0.95(\mathrm{~d}, J=7.5 \mathrm{~Hz}, 3 \mathrm{H}), 0.90(\mathrm{t}, J=3.0 \mathrm{~Hz}, 3 \mathrm{H}), 0.88(\mathrm{~s}, 9 \mathrm{H}), 0.04(\mathrm{~s}, 6 \mathrm{H})$; ${ }^{13} \mathrm{C}$ NMR $\left(125 \mathrm{MHz}, \mathrm{CDCl}_{3}\right) \delta 78.3,68.7,61.2,56.9,40.6,40.5,40.2,39.0,26.6,26.2,20.0$, 19.1, 18.5, 14.4, -5.0; LRMS (ESI): Mass calcd for $\mathrm{C}_{36} \mathrm{H}_{82} \mathrm{O}_{7} \mathrm{Si}\left[2 \mathrm{M}+\mathrm{H}_{2} \mathrm{O}\right]^{+}$, 683. Found $[2 \mathrm{M}+$ $\left.\mathrm{H}_{2} \mathrm{O}\right], 682 ;[\alpha]_{\mathrm{D}}^{25}=-14.8\left(\mathrm{CHCl}_{3}, \mathrm{c}=0.62\right)$.

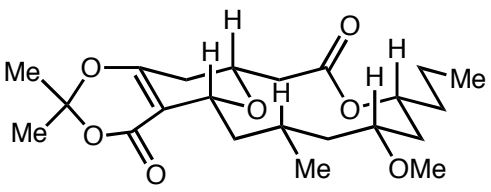

Tricyclic Dioxinone (15): To round bottom charged with aldehyde 4 (21.6 $\mathrm{mg}, 0.05 \mathrm{mmol})$, $\mathrm{CaSO}_{4}(20.6 \mathrm{mg}, 1.52 \mathrm{mmol})$, and $\mathrm{Sc}(\mathrm{OTf})_{3}(4.9 \mathrm{mg}, 0.01 \mathrm{mmol})$ was added $\mathrm{MeCN}(5.0 \mathrm{~mL})$. The reaction stirred for $45 \mathrm{~min}$ and then brine $(0.5 \mathrm{~mL})$ was added and the reaction mixture was filtered through a short pad of Celite eluting with $\mathrm{CH}_{2} \mathrm{Cl}_{2}$. The layers were separated and the aqueous layer was extracted with EtOAc $(4 \mathrm{X} 5 \mathrm{~mL})$. The combined organic layers were dried with anhydrous $\mathrm{Na}_{2} \mathrm{SO}_{4}$, filtered and concentrated. The resulting residue was purified by flash column chromatography (30\% EtOAc:hexanes) to afford tricyclic dioxinone $15(5.0 \mathrm{mg}, 25 \%)$ as a colorless oil. Analytical data for 15: ${ }^{1} \mathrm{H} \mathrm{NMR}\left(500 \mathrm{MHz}, \mathrm{CDCl}_{3}\right) \delta 5.21(\mathrm{~m}, 1 \mathrm{H}), 4.44(\mathrm{~d}, J=$ 10.0, 1H), $4.04(\mathrm{~m}, 1 \mathrm{H}), 3.35(\mathrm{~s}, 3 \mathrm{H}), 3.22(\mathrm{~m}, 1 \mathrm{H}), 2.53(\mathrm{dd}, J=12 ., 2.5 \mathrm{~Hz}, 1 \mathrm{H}), 2.47(\mathrm{dd}, J=$ 11.0, 11.0, 1H), 2.35-2.11 (m, 4H), $1.82(\mathrm{~d}, J=7.5 \mathrm{~Hz}, 1 \mathrm{H}), 1.70(\mathrm{~s}, 6 \mathrm{H}), 1.59-1.48(\mathrm{~m}, 4 \mathrm{H}), 1.35$ 
(ddd, $J=14.0,9.5,6.0 \mathrm{~Hz}, 2 \mathrm{H}), 1.22-1.17(\mathrm{~m}, 2 \mathrm{H}), 1.03(\mathrm{~d}, J=7.5 \mathrm{~Hz}, 3 \mathrm{H}), 0.93(\mathrm{dd}, J=7.5$, $7.5 \mathrm{~Hz}, 3 \mathrm{H}) ;{ }^{13} \mathrm{C}$ NMR $\left(125 \mathrm{MHz}, \mathrm{CDCl}_{3}\right) \delta 171.7,162.2,159.5,106.0,105.9,82.5,76.0,75.1$, 71.0, 56.9, 44.9, 42.5, 41.9, 40.5, 37.9, 33.6, 33.3, 27.7, 25.3, 22.1, 18.7, 14.1; LRMS (ESI): Mass calcd for $\mathrm{C}_{44} \mathrm{H}_{68} \mathrm{O}_{14} \mathrm{Na}[2 \mathrm{M}+\mathrm{Na}]^{+}, 843$. Found $[2 \mathrm{M}+\mathrm{Na}]^{+}, 843$.

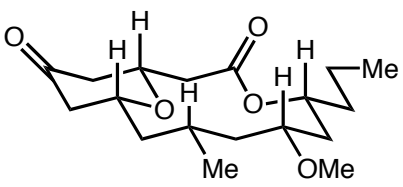

(1R,5R,7R,9S,11R)-7-methoxy-9-methyl-5-propyl-4,15-dioxabicylo[9.3.1]penta-decane3,12-dione (28): To a one-dram vial charged with tricyclic dioxinone 15 (4.1 $\mathrm{mg}, 0.01 \mathrm{mmol})$ was added DMSO $(0.18 \mathrm{~mL})$ and $\mathrm{H}_{2} \mathrm{O}(0.5 \mathrm{~mL})$. The reaction was then submerged into a preheated oil bath of $130{ }^{\circ} \mathrm{C}$. The reaction stirred for $12 \mathrm{hrs}$ and was then diluted with EtOAc (2 $\mathrm{mL})$ and added to a separatory funnel containing brine $(120 \mathrm{~mL})$. The layers were separated and the aqueous layer was extracted with EtOAc $(5 \mathrm{X} 5 \mathrm{~mL})$. The combined organic layers were dried with anhydrous $\mathrm{Na}_{2} \mathrm{SO}_{4}$, filtered and concentrated. The resulting residue was purified by flash column chromatography (30\% EtOAc:hexanes) to afford pyranone $\mathbf{2 8}(3.2 \mathrm{mg}, 99 \%)$ as a colorless oil. Analytical data for 28: IR (film) 2918, 2872, 1724, 1651, 1558, 1369, $1100 \mathrm{~cm}^{-1}$; ${ }^{1} \mathrm{H}$ NMR $\left(500 \mathrm{MHz}, \mathrm{CDCl}_{3}\right) \delta 5.26$ (dddd, $\left.J=11.0,5.5,5.5,5.5,1 \mathrm{H}\right), 4.01$ (dd, $J=11.0,11.0$, $1 \mathrm{H}), 3.71(\mathrm{dd}, J=10.5,10.5,1 \mathrm{H}), 3.33(\mathrm{~s}, 3 \mathrm{H}), 3.21(\mathrm{dd}, J=8.5,8.5,1 \mathrm{H}), 2.55(\mathrm{dd}, J=11.5$, $11.5,1 \mathrm{H}), 2.45(\mathrm{~d}, J=13.5,2 \mathrm{H}), 2.36(\mathrm{~d}, J=14.0,2 \mathrm{H}), 2.26(\mathrm{ddd}, J=16.0,16.0,12.5,2 \mathrm{H}), 1.84$ $(\mathrm{d}, J=15.0,1 \mathrm{H}), 1.65-1.53(\mathrm{~m}, 3 \mathrm{H}), 1.51-1.46(\mathrm{~m}, 2 \mathrm{H}), 1.38-1.32(\mathrm{~m}, 3 \mathrm{H}), 1.18(\mathrm{ddd}, J=14.5$, 9.5, 6.5, 1H), $1.03(\mathrm{~d}, J=6.0,3 \mathrm{H}), 0.93(\mathrm{dd}, J=8.0,3 \mathrm{H}) ;{ }^{13} \mathrm{C} \mathrm{NMR}\left(125 \mathrm{MHz}, \mathrm{CDCl}_{3}\right) \delta 205.9$, 171.9, 83.3, 79.5, 75.4, 75.0, 56.5, 48.6, 47.5, 44.8, 44.7, 42.7, 39.9, 38.0, 34.5, 24.6, 18.8, 14.1; LRMS (ESI): Mass calcd for $\mathrm{C}_{36} \mathrm{H}_{60} \mathrm{O}_{10} \mathrm{Na}[2 \mathrm{M}+\mathrm{Na}]^{+}, 676$. Found $[2 \mathrm{M}+\mathrm{Na}]^{+}, 676 .[\alpha]_{\mathrm{D}}{ }^{25}=$ $+20.6\left(\mathrm{CHCl}_{3}, \mathrm{c}=0.1\right)$.

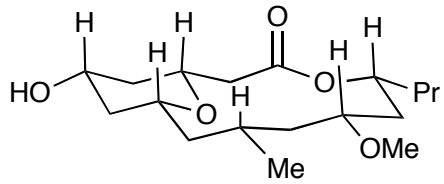

$(1 R, 5 R, 7 R, 9 S, 11 R, 13 S)-13-h y d r o x y-7-m e t h o x y-9-m e t h y l-5-p r o p y l-4,15-$

dioxabicyclo[9.3.1]pentadecan-3-one (2): To a one dram vial charged with pyranone 28 (4.2 $\mathrm{mg}, 0.013 \mathrm{mmol})$ was added $\mathrm{MeOH}(0.26 \mathrm{~mL})$. The reaction mixture was cooled to $0{ }^{\circ} \mathrm{C}$ and $\mathrm{NaBH}_{4}(1.0 \mathrm{mg}, 0.026 \mathrm{mmol})$ was added. After $10 \mathrm{~min}$, AcOH $(8.0 \mu \mathrm{L})$ was added and the reaction was concentrated in vacuo. The resulting residue was purified by flash column chromatography (50\% EtOAc:hexanes) to afford alcohol $2(2.9 \mathrm{mg}, 96 \%)$ as a colorless oil. Analytical data for 2: ${ }^{1} \mathrm{H}$ NMR $\left(500 \mathrm{MHz}, \mathrm{CDCl}_{3}\right) \delta 5.25(\mathrm{~m}, 1 \mathrm{H}), 3.84$ (dddd, $J=5.8,5.8,5.8$, $5.8,1 \mathrm{H}), 3.74$ (apt, $J=10.9,1 \mathrm{H}), 3.43(\mathrm{apt}, J=10.6,1 \mathrm{H}), 3.43(\mathrm{~s}, 3 \mathrm{H}), 3.21$ (apt, $J=8.4,1 \mathrm{H})$, $2.43(\mathrm{ddd}, J=14.7,3.6,3.6,2 \mathrm{H}), 2.29(\mathrm{~d}, J=14.4,1 \mathrm{H}), 2.02(\mathrm{dd}, J=9.8,2.0,1 \mathrm{H}), 1.95(\mathrm{dd}, J=$ $4.2,2.0,1 \mathrm{H}), 1.82(\mathrm{~d}, J=14.8,1 \mathrm{H}), 1.63-1.44(\mathrm{~m}, 7 \mathrm{H}), 1.43-1.13(\mathrm{~m}, 7 \mathrm{H}), 1.00(\mathrm{~d}, J=6.7,3 \mathrm{H})$, $0.93(\mathrm{dd}, J=7.3,3 \mathrm{H}) ;{ }^{13} \mathrm{C} \mathrm{NMR}\left(125 \mathrm{MHz}, \mathrm{CDCl}_{3}\right) \delta 172.8,83.3,78.3,74.7,74.2,68.0,56.5$, $45.1,44.5,42.6,41.9,40.9,40.0,38.0,34.5,24.8,18.8,14.1$; 


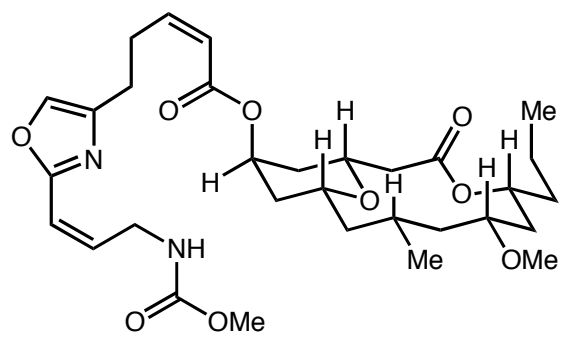

Originally proposed structure (1): To a one dram vial charged with alcohol $2(2.0 \mathrm{mg}, 0.006$ mmol), oxazole $5(6.1 \mathrm{mg}, 0.022 \mathrm{mmol})$ and $\mathrm{PPh}_{3}(6.22 \mathrm{mg}, 0.024 \mathrm{mmol})$ to which was added benzene $(0.33 \mathrm{~mL})$. To the reaction mixture was added diisopropyl azodicarboxylate $(4.7 \mu \mathrm{L}$, $0.024 \mathrm{mmol})$. After five min the reaction was concentrated in vacuo. The resulting residue was purified by flash column chromatography (30\% EtOAc:hexanes) to afford 1 (2.6 mg, 73\%) as a colorless oil. Analytical data for 1: IR (film) 3361, 2922, 2871, 1718, 1649, 1520, 1459, 1248, 1159, $1106 \mathrm{~cm}^{-1} ;{ }^{1} \mathrm{H}$ NMR (600 MHz, CD $\left.3 \mathrm{OD}\right) \delta 7.65$ (s, 1H), 6.39 (ddd, $J=11.5,7.4,7.4,1 \mathrm{H}$ ), 6.33 (ddd, $J=11.8,2.0,2.0,1 \mathrm{H}), 6.05$ (ddd, $J=12.1,6.1,6.1,1 \mathrm{H}), 5.89$ (ddd, 11.5, 1.5, 1.5, $1 \mathrm{H}), 5.26-5.23(\mathrm{~m}, 1 \mathrm{H}), 5.21$ (dddd, $J=2.9,2.9,2.9,2.9,1 \mathrm{H}), 4.32(\mathrm{~d}, J=4.8,2 \mathrm{H}), 4.03$ (ddd, $J$ $=13.2,4.5,2.1,1 \mathrm{H}), 3.71(\mathrm{dd}, J=11.4,1 \mathrm{H}), 3.6(\mathrm{~s}, 3 \mathrm{H}), 3.3(\mathrm{~s}, 3 \mathrm{H}), 3.24(\mathrm{dd}, J=8.1,8.1,1 \mathrm{H})$, $3.02(\mathrm{ddd}, J=7.5,7.5,2 \mathrm{H}), 2.73(\mathrm{dd}, J=8.0,8.0,2 \mathrm{H}), 2.37(\mathrm{dd}, J=12.3,2.9,1 \mathrm{H}), 2.33(\mathrm{dd}, J=$ $17.3,6.5,1 \mathrm{H}), 1.85-1.81(\mathrm{~m}, 2 \mathrm{H}), 1.72$ (apdd, $J=14.3,2.7,1 \mathrm{H}), 1.58-1.47(\mathrm{~m}, 6 \mathrm{H}), 1.39-1.20$ $(\mathrm{m}, 6 \mathrm{H}), 1.06(\mathrm{ddd}, J=14.5,10.1,6.6,1 \mathrm{H}), 0.96(\mathrm{~d}, J=6.9,3 \mathrm{H}), 0.93(\mathrm{dd}, J=7.3,3 \mathrm{H}) ;{ }^{13} \mathrm{C}$ NMR $\left(125 \mathrm{MHz}, \mathrm{CD}_{3} \mathrm{OD}\right) \delta$ 173.4, 165.6, 160.6, 158.4, 148.8, 141.0, 137.9, 134.7, 120.4, 114.7, 83.2, 75.2, 74.4, 71.5, 67.7, 55.3, 51.3, 44.8, 44.3, 41.9, 40.0, 39.7, 37.5, 35.8, 34.8, 34.3, 27.7, 25.1, 23.9, 18.4, 12.9; LRMS (ESI): Mass calcd for $\mathrm{C}_{31} \mathrm{H}_{47} \mathrm{~N}_{2} \mathrm{O}_{9}[\mathrm{M}+\mathrm{H}]^{+}$, 591. Found [M+H] $]^{+}$, 591. $\left.[\alpha]_{\mathrm{D}}^{25}=+19.0\left(\mathrm{CH}_{3} \mathrm{OH}\right), \mathrm{c}=0.03\right)$.

\section{Characterization Data for Diastereomer 17}

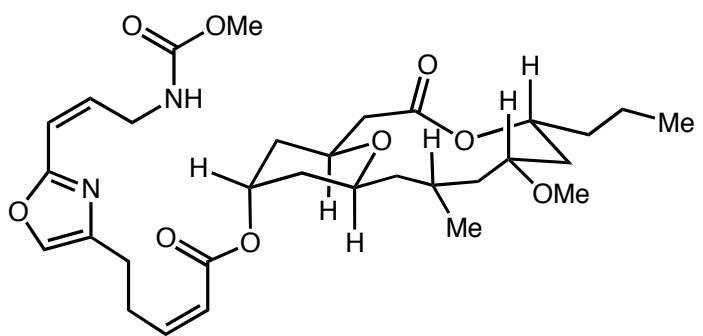

Analytic data for 17: ${ }^{1} \mathrm{H} \mathrm{NMR}\left(500 \mathrm{MHz}, \mathrm{CD}_{3} \mathrm{OD}\right) \delta 6.34$ (ddd, $\left.J=11.7,7.6,7.6,1 \mathrm{H}\right), 6.25$ (apd, $J=11.7,1 \mathrm{H}), 6.02(\mathrm{ddd}, J=11.7,5.8,5.8,1 \mathrm{H}), 5.86(\mathrm{apd}, J=11.7,1 \mathrm{H}), 5.21(\mathrm{~m}, 1 \mathrm{H})$, $5.11(\mathrm{~m}, 1 \mathrm{H}), 4.28(\mathrm{~d}, J=5.2,2 \mathrm{H}), 4.01(\mathrm{~m}, 1 \mathrm{H}), 3.74(\mathrm{apt}, J=10.5,1 \mathrm{H}), 3.63(\mathrm{~s}, 3 \mathrm{H}), 3.32(\mathrm{~s}$, $3 \mathrm{H}), 2.99$ (ddd, $J=7.0,7.0,7.0,2 \mathrm{H}), 2.69$ (dd, $J=7.6,2 \mathrm{H}), 2.65(\mathrm{ddd}, J=4.1,4.1,4.1,1 \mathrm{H})$, 2.24 (ddd, $J=14.0,8.7,1 \mathrm{H}), 1.90-1.74(\mathrm{~m}, 4 \mathrm{H}), 1.67-1.50(\mathrm{~m}, 7 \mathrm{H}), 1.34-1.09(\mathrm{~m}, 7 \mathrm{H}), 0.91(\mathrm{~d}, J$ $=7.6,3 \mathrm{H}), 0.88(\mathrm{dd}, J=7.0,3 \mathrm{H})$; LRMS (ESI): Mass calcd for $\mathrm{C}_{31} \mathrm{H}_{47} \mathrm{~N}_{2} \mathrm{O}_{9}[\mathrm{M}+1]^{+}, 591$. Found $[\mathrm{M}+\mathrm{H}]^{+}, 591$.

\section{Experimental Procedures and Characterization Data for Synthesis of Revised Structure}


Alcohol 18 could in theory be prepared from the sequence outlined above beginning from the enantiomer of $\beta$-hydroxy amide 10. However, given the stereochemical discrepancies between our synthetic $\mathbf{1}$ and the natural product we chose to start from amide $\mathbf{1 0}$ followed by robust reactions with defined stereochemical results.

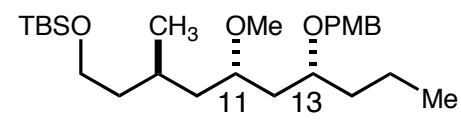

tert-Butyl((3R,5S,7R)-5-methoxy-7-(4-methoxybenzyloxy)-3-methyldecyloxy) dimethylsilane (29): Iodide $12(0.126 \mathrm{~g}, 0.385 \mathrm{mmol})$ was dried by azeotroping with benzene 3 times then dissolved in pentane (freshly distilled from $\mathrm{CaH}_{2}, 3 \mathrm{~mL}$ ) and ether $(2 \mathrm{~mL})$ and cooled to $-78{ }^{\circ} \mathrm{C}$. A solution of $t$-butyllithium $(2.7 \mathrm{M}$ in heptane, $0.30 \mathrm{~mL})$ was added dropwise and the mixture was stirred at $-78{ }^{\circ} \mathrm{C}$ for $15 \mathrm{~min}$, then warmed to $0{ }^{\circ} \mathrm{C}$ for $20 \mathrm{~min}$ (after which a white precipitate was noticed). The solution was cooled back down to $-78{ }^{\circ} \mathrm{C}$ and a solution of $(R)-3-(4-$ methoxybenzyloxy)hexanal (derived from the DIBAL reduction of amide 11) $(0.091 \mathrm{~g}, 0.39$ mmol) in THF was cooled to $-78{ }^{\circ} \mathrm{C}$ and added via cannula (dripped along the side of the cooled reaction flask). This mixture was stirred for $15 \mathrm{~min}$ at $-78{ }^{\circ} \mathrm{C}$. The reaction was quenched at low temperature with the addition of saturated aqueous $\mathrm{NH}_{4} \mathrm{Cl}(50 \mathrm{~mL})$. Upon warming to ambient temperature, the mixture was extracted with EtOAc $(3 \times 50 \mathrm{~mL})$, the organic layers combined, dried over anhydrous $\mathrm{Na}_{2} \mathrm{SO}_{4}$, filtered and concentrated. This mixture of $\mathrm{C} 11 / \mathrm{C} 13$ syn- and anti-addition products proved difficult to separate and was carried forward without exhaustive separation.

The alcohol $(1: 1$ mixture of diastereomers, $0.300 \mathrm{~g}, 0.685 \mathrm{mmol})$ was dissolved in $\mathrm{CH}_{2} \mathrm{Cl}_{2}(2 \mathrm{~mL})$ and 2,6-di-tert-butyl-4-methylpyridne $(0.703 \mathrm{~g}, 3.42 \mathrm{mmol})$ was added. This solution was cooled to $0{ }^{\circ} \mathrm{C}$ and freshly prepared MeOTf $(0.37 \mathrm{~mL}, 3.42 \mathrm{mmol})$ added dropwise. The ice bath was then removed and the mixture stirred for $12 \mathrm{~h}$ at $23{ }^{\circ} \mathrm{C}$ (formation of a white precipitate observed). After $12 \mathrm{~h}$, a second portion of MeOTf $(0.37 \mathrm{~mL}, 3.42 \mathrm{mmol})$ was added and the mixture was allowed to stir for an additional $8 \mathrm{~h}$. The mixture was then concentrated in vacuo and the residue was purified by flash column chromatography (5\% EtOAc/hexanes) to afford methyl ether $29(0.093 \mathrm{~g}, 30 \%)$ as a colorless oil. Analytical data for 29: IR (film) 2931, $2859,2362,1614,1514,1465,1249,1095,835,776 \mathrm{~cm}^{-1} ;{ }^{1} \mathrm{H}$ NMR $\left(500 \mathrm{MHz}, \mathrm{CDCl}_{3}\right) \delta 7.26(\mathrm{~d}$, $J=8.5 \mathrm{~Hz}, 2 \mathrm{H}), 6.86(\mathrm{~d}, J=8.5 \mathrm{~Hz}, 2 \mathrm{H}), 4.45\left(\mathrm{~A}\right.$ of ABq, $\left.J_{\mathrm{AB}}=11.0 \mathrm{~Hz}, 1 \mathrm{H}\right), 4.39 \mathrm{~B}$ of ABq, $\left.J_{\mathrm{AB}}=11.5 \mathrm{~Hz}, 1 \mathrm{H}\right), 3.80(\mathrm{~s}, 3 \mathrm{H}), 3.63(\mathrm{ddd}, J=18.5,18.5,6.0 \mathrm{~Hz}, 2 \mathrm{H}), 3.45$ (dddd, $J=11.0,5.0$, $5.0,5.0 \mathrm{~Hz}, 1 \mathrm{H}), 3.37(\mathrm{~m}, 1 \mathrm{H}), 3.29(\mathrm{~s}, 3 \mathrm{H}), 1.90(\mathrm{ddd}, J=13.5,6.0,6.0 \mathrm{~Hz}, 1 \mathrm{H}), 1.74(\mathrm{~m}, 1 \mathrm{H})$, $1.57-1.46(\mathrm{~m}, 5 \mathrm{H}), 1.45-1.25(\mathrm{~m}, 3 \mathrm{H}) 1.19$ (ddd, $J=13.0,8.5,4.0 \mathrm{~Hz}, 1 \mathrm{H}), 0.92(\mathrm{~d}, J=7.5 \mathrm{~Hz}$, $3 \mathrm{H}), 0.90-0.87(\mathrm{~m}, 3 \mathrm{H}), 0.89$ (s, 9H), $0.04(\mathrm{~s}, 6 \mathrm{H}) ;{ }^{13} \mathrm{C} \mathrm{NMR}\left(125 \mathrm{MHz}, \mathrm{CDCl}_{3}\right) \delta 159.3,131.2$, 129.6, 113.9, 76.3, 75.8, 70.5, 61.6, 56.3, 55.5, 42.3, 40.8, 39.0, 36.6, 26.4, 26.2, 19.9, 18.7, 18.5, 14.5, -5.0; LRMS (ESI): Mass calcd for $\mathrm{C}_{26} \mathrm{H}_{48} \mathrm{O}_{4} \mathrm{SiNa}$ [M+Na] ${ }^{+}$, 476. Found [M+Na], 476; $[\alpha]_{D}^{25}=-16.3\left(\mathrm{CHCl}_{3}, \mathrm{c}=0.49\right)$.

Stereochemical proof of $\mathrm{C} 11 / \mathrm{C} 13$ cis relationship of 29 
<smiles>COc1ccc([C@H]2O[C@@H](CC(C)C)C[C@@H](CC[C@H](C)CCOC(C)(C)C)O2)cc1</smiles>

tert-butyl $((R)-4-((2 R, 4 S, 6 R)-2-(4-m e t h o x y p h e n y l)-6-p r o p y l-1,3-d i o x a n-4-y l)-3-$

methylbutoxy)dimethylsilane (30): The cis alcohol from the addition above was separated after multiple purifications by column chromatography. A pure sample of this material $(0.0150 \mathrm{~g}$, $0.0355 \mathrm{mmol})$ was dissolved in $\mathrm{CH}_{2} \mathrm{Cl}_{2}(0.5 \mathrm{~mL})$, activated $4 \AA$ molecular sieves $(0.0249 \mathrm{~g})$ and the mixture cooled to $0{ }^{\circ} \mathrm{C}$. DDQ $(0.0121 \mathrm{~g}, 0.533 \mathrm{mmol})$ added and the mixture stirred for $2 \mathrm{~h}$ while warming to $23{ }^{\circ} \mathrm{C}$. The reaction was quenched by the addition of saturated aqueous $\mathrm{NaHCO}_{3}(3 \mathrm{~mL})$, and the aqueous phase was extracted with $\mathrm{CH}_{2} \mathrm{Cl}_{2}(3 \times 5 \mathrm{~mL})$. The organic layers were combined, dried over anhydrous $\mathrm{Na}_{2} \mathrm{SO}_{4}$, filtered and concentrated. The residue was purified by flash column chromatography (5\% acetone/hexanes) to afford acetal $30(0.011 \mathrm{~g}$, 75\%) as a colorless oil. Analytical data for 30: IR (film) 2928, 2856, 2362, 2336, 1650, 1514, 1464, 1249, 1094, $1035 \mathrm{~cm}^{-1} ;{ }^{1} \mathrm{H}$ NMR $\left(500 \mathrm{MHz}, \mathrm{CDCl}_{3}\right) \delta 7.42(\mathrm{~d}, J=8.5 \mathrm{~Hz}, 2 \mathrm{H}), 6.87(\mathrm{~d}, J$ $=9.0 \mathrm{~Hz}, 2 \mathrm{H}), 5.46(\mathrm{~s}, 1 \mathrm{H}), 3.88(\mathrm{~m}, 1 \mathrm{H}), 3.79(\mathrm{~m}, 4 \mathrm{H}), 3.65(\mathrm{dddd}, J=13.0,9.5,9.5,6.0,2 \mathrm{H})$, 1.73-1.57 (m, 6H), 1.53-1.34 (m, 5H), 1.29-1.24 (m, 2H), 0.95-0.92 (m, 6H), $0.87(\mathrm{~s}, 9 \mathrm{H}), 0.04$ $(\mathrm{s}, 6 \mathrm{H}) ;{ }^{13} \mathrm{C}$ NMR $\left(125 \mathrm{MHz}, \mathrm{CDCl}_{3}\right) \delta 159.8,132.0,127.5,113.6,100.5,76.8,74.8,61.5,55.5$, 43.7, 40.5, 38.4, 37.7, 26.2, 25.7, 19.8, 18.6, 18.6, 14.3, -5.1; LRMS (ESI): Mass calcd for $\mathrm{C}_{25} \mathrm{H}_{44} \mathrm{O}_{4} \mathrm{Si}[\mathrm{M}]^{+}, 437$. Found $[\mathrm{M}]^{+}, 437 ;[\alpha]_{\mathrm{D}}^{25}=-5.0\left(\mathrm{CHCl}_{3}, \mathrm{c}=0.14\right)$.

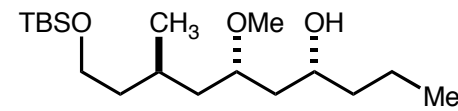

(4R,6S,8R)-10-(tert-butyldimethylsilyloxy)-6-methoxy-8-methyldecan-4-ol (31): PMB ether 29 (0.0967 g, $0.214 \mathrm{mmol}$ ) was dissolved in $\mathrm{CH}_{2} \mathrm{Cl}_{2}(20 \mathrm{~mL}), \mathrm{pH} 7$ phosphate buffer added (2 $\mathrm{mL})$ and the mixture cooled to $0{ }^{\circ} \mathrm{C}$. DDQ was added $(0.0582 \mathrm{~g}, 0.26 \mathrm{mmol})$ and the mixture stirred for $3 \mathrm{~h}$ at $0{ }^{\circ} \mathrm{C}$. The reaction was quenched by the addition of saturated aqueous $\mathrm{NaHCO}_{3}$ $(20 \mathrm{~mL})$, and the aqueous phase was extracted with $\mathrm{CH}_{2} \mathrm{Cl}_{2}(3 \times 30 \mathrm{~mL})$. The organic layers were combined, dried over anhydrous $\mathrm{Na}_{2} \mathrm{SO}_{4}$, filtered and concentrated. The residue was purified by flash column chromatography (6\%-8\% gradient EtOAc/hexanes) to afford alcohol 31 $(0.059 \mathrm{~g}, 83 \%)$ as a colorless oil. Analytical data for 31: IR (film) 3447, 2956, 2932, 2864, 2362, 2336, 1465, 1380, 1253, 1095, $836 \mathrm{~cm}^{-1}$; ${ }^{1} \mathrm{H}$ NMR (500 MHz, $\left.\mathrm{CDCl}_{3}\right) \delta 3.77(\mathrm{~m}, 1 \mathrm{H}), 3.68-3.60$ $(\mathrm{m}, 2 \mathrm{H}), 3.57(\mathrm{~s}, 1 \mathrm{H}), 3.51(\mathrm{~m}, 1 \mathrm{H}), 3.35(\mathrm{~s}, 3 \mathrm{H}), 1.69(\mathrm{~m}, 1 \mathrm{H}), 1.63-1.52(\mathrm{~m}, 4 \mathrm{H}), 1.49-1.42(\mathrm{~m}$, 2H), 1.39-1.28 (m, 3H), 1.25 (ddd, $J=21.0,14.0,7.0 \mathrm{~Hz}, 1 \mathrm{H}), 0.93-0.92(\mathrm{~m}, 6 \mathrm{H}), 0.88$ (s, 9H), 0.04 (s, 6H); ${ }^{13} \mathrm{C}$ NMR (125 MHz, $\left.\mathrm{CDCl}_{3}\right) \delta$ 80.4, 71.8, 61.2, 56.2, 41.6, 41.4, 40.2, 40.1, 26.3, 26.2, 20.5, 18.9, 18.5, 14.4, -5.2; LRMS (ESI): Mass calcd for $\mathrm{C}_{18} \mathrm{H}_{40} \mathrm{O}_{3} \mathrm{Si}[\mathrm{M}]^{+}$, 333. Found $[\mathrm{M}]^{+}, 333 ;[\alpha]_{\mathrm{D}}{ }^{25}=+16.3\left(\mathrm{CHCl}_{3}, \mathrm{c}=0.27\right)$. 


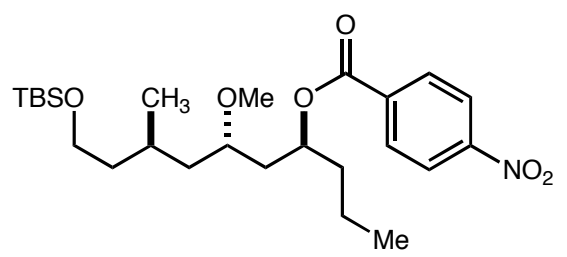

(4S,6S,8R)-10-(tert-butyldimethylsilyloxy)-6-methoxy-8-methyldecan-4-yl-4-nitrobenzoate

32: To a round bottom charged with alcohol $31(59 \mathrm{mg}, 0.118 \mathrm{mmol})$ was added benzene (2.0 $\mathrm{mL}$ ) followed by addition of $\mathrm{PPh}_{3}(24 \mathrm{mg}, 0.90 \mathrm{mmol})$, 4-nitrobenzoic acid $(135 \mathrm{mg}, 0.81 \mathrm{mmol})$ and diethyl azodicarboxylate $(141 \mu \mathrm{L}, 0.90 \mathrm{mmol})$. The reaction stirred for three $\mathrm{h}$ and was diluted with EtOAc $(10 \mathrm{~mL})$. The mixture was then washed with water $(10 \mathrm{~mL})$ and then brine $(10 \mathrm{~mL})$. The combined aqueous layers were extracted with EtOAc $(2 \times 10 \mathrm{~mL})$. The combined organic layers were dried with anhydrous $\mathrm{Na}_{2} \mathrm{SO}_{4}$, filtered and concentrated. The resulting residue was purified by flash column chromatography (5\% EtOAc:hexanes) to afford ester 32 (63 mg, 73\%) of a colorless oil. Analytical data for 32: IR (film) 2930, 2858, 1724, 1530, 1724, $1530,1274,1097,836,717 \mathrm{~cm}^{-1} ;{ }^{1} \mathrm{H}$ NMR $\left(500 \mathrm{MHz}, \mathrm{CDCl}_{3}\right) \delta 8.30(\mathrm{~d}, J=9.0,2 \mathrm{H}), 8.23(\mathrm{~d}, J$ $=9.0,2 \mathrm{H}), 5.41(\mathrm{dddd}, J=8.5,8.5,8.5,8.5,1 \mathrm{H}), 3.63(\mathrm{ddd}, J=13.0,13.0,10.5,2 \mathrm{H}), 3.34-3.32$ (m, $1 \mathrm{H}), 3.29(\mathrm{~s}, 3 \mathrm{H}), 1.87$ (ddd, $J=14.5,9.0,3.5,1 \mathrm{H}), 1.81-1.60(\mathrm{~m}, 5 \mathrm{H}), 1.56$ (ddd, $J=13.0$, 13.0, 7.0, 1H), 1.46-1.37 (m, 2H), 1.28 (ddd, $J=20.5,14.0,7.0,1 \mathrm{H}), 1.22$ (ddd, $J=13.5,6.0$, $6.0,1 \mathrm{H}), 0.95(\mathrm{dd}, J=7.5,7.5,3 \mathrm{H}), 0.91(\mathrm{~d}, J=6.0,3 \mathrm{H}), 0.88(\mathrm{~s}, 9 \mathrm{H}), 0.04(\mathrm{~s}, 6 \mathrm{H}) ;{ }^{13} \mathrm{C}$ NMR $\left(125 \mathrm{MHz}, \mathrm{CDCl}_{3}\right) \delta 164.5,150.6,136.3,130.8,123.7,84.3,75.9,74.1,73.7,61.2,57.0,42.1$, 40.2, 39.7, 37.4, 26.4, 26.1, 20.2, 18.7, 18.5, 14.2, -5.0; LRMS (ESI): Mass calcd for $\mathrm{C}_{25} \mathrm{H}_{4} \mathrm{NO}_{5} \mathrm{Si}[\mathrm{M}]^{+}, 482$. Found $[\mathrm{M}]^{+}, 482 .[\alpha]_{\mathrm{D}}^{25}=+19.1\left(\mathrm{CHCl}_{3}, \mathrm{c}=0.83\right)$.

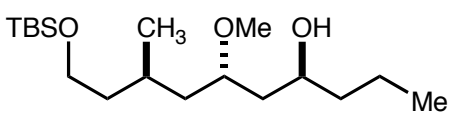

(4S,6S,8R)-10-(tert-butyldimethylsilyloxy)-6-methoxy-8-methyldecan-4-ol 18: To a round bottom flask charged with ester $32(63 \mathrm{mg}, 0.13 \mathrm{mmol})$ was added $\mathrm{MeOH}(2 \mathrm{~mL})$ followed by $\mathrm{K}_{2} \mathrm{CO}_{3}(898 \mathrm{mg}, 6.5 \mathrm{mmol})$. The reaction mixture stirred for $3 \mathrm{~h}$ and was then concentrated in vacuo. The resulting residue was purified by flash column chromatography (10\% EtOAc:hexanes) to afford alcohol 18 (30 mg, 70\%) as a colorless oil. Analytical data for 18: IR (film) 3446, 2931, 2860, 1650, 1461, 1253, 1094, $836 \mathrm{~cm}^{-1}$; ${ }^{1} \mathrm{H}$ NMR $\left(500 \mathrm{MHz}, \mathrm{CDCl}_{3}\right) \delta 3.91$ $(\mathrm{m}, 1 \mathrm{H}), 3.66(\mathrm{~m}, 2 \mathrm{H}), 3.60$ (dddd, $J=6.0,6.0,6.0,6.0,1 \mathrm{H}), 1.77-1.69(\mathrm{~m}, 3 \mathrm{H}), 1.63-1.44(\mathrm{~m}$, $6 \mathrm{H}), 1.40-1.31(\mathrm{~m}, 4 \mathrm{H}), 1.24$ (ddd, $J=15.5,9.0,5.0,2 \mathrm{H}), 0.96-0.93(\mathrm{~m}, 6 \mathrm{H}), 0.90(\mathrm{~s}, 9 \mathrm{H}), 0.06$ (s, 6H); ${ }^{13} \mathrm{C}$ NMR $\left(125 \mathrm{MHz}, \mathrm{CDCl}_{3}\right) \delta 78.0,68.7,61.3,56.9,47.6,41.2,40.2,39.4,26.6,26.2$, 20.2, 19.1, 18.5, 14.4, -5.1; LRMS (ESI): Mass calcd for $\mathrm{C}_{18} \mathrm{H}_{40} \mathrm{O}_{3} \mathrm{Si}[\mathrm{M}]^{+}$, 333. Found $[\mathrm{M}]^{+}$, 333. $[\alpha]_{\mathrm{D}}^{25}=+10.9\left(\mathrm{CHCl}_{3}, \mathrm{c}=0.3\right)$.

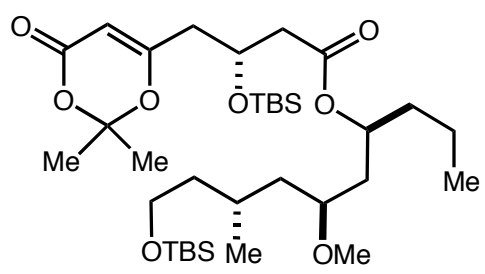

$(R)$-((4S,6S,8R)-10-(tert-butyldimethylsilyloxy)-6-methoxy-8-methyldecan-4-yl)-3-(tertbutyldimethylsilyloxy)-4-(2,2-dimethyl-4-oxo-4H-1,3-dioxin-6-yl)butanoate (33): $\quad$ To a 
solution of carboxylic acid $5(53.9 \mathrm{mg}, 0.156 \mathrm{mmol})$ in THF $(2.8 \mathrm{~mL})$ was added $\mathrm{Et}_{3} \mathrm{~N}(23 \mu \mathrm{L}$, $0.166 \mathrm{mmol})$ followed by 2,4,6-trichlorobenzoyl chloride $(25 \mu \mathrm{L}, 0.156 \mathrm{mmol})$. The reaction mixture was allowed to stir for one hour and a mixture of alcohol 18 (52 $\mathrm{mg}, 0.156 \mathrm{mmol}$ ), DMAP (21 mg, $0.166 \mathrm{mmol})$ in THF $(0.8 \mathrm{~mL})$ was added via cannula. The reaction was allowed to stir for 18 hours and was then quenched by the addition of saturated aqueous $\mathrm{NH}_{4} \mathrm{Cl}$. The aqueous layer was separated and extracted with EtOAc (4 X $2 \mathrm{~mL})$. The combined organic layers were dried with anhydrous $\mathrm{Na}_{2} \mathrm{SO}_{4}$, filtered and concentrated. The resulting residue was purified by flash column chromatography to afford $\mathbf{3 3}(81.5 \mathrm{~g}, 82 \%)$ as a clear oil. Analytical data for 33: IR (film) $\mathrm{cm}^{-1}$; ${ }^{1} \mathrm{H}$ NMR $\left(500 \mathrm{MHz} \mathrm{CDCl}_{3}\right) \delta 5.30(\mathrm{~s}, 1 \mathrm{H}), 5.10$ (dddd, $J=12.5,6.0$, $6.0,6.0,1 \mathrm{H}), 4.41$ (dddd, $J=12.0,6.0,6.0,6.0,1 \mathrm{H}), 3.65$ (ddd, $J=17.5,17.5,10.0,2 \mathrm{H}), 3.29$ $(\mathrm{s}, 3 \mathrm{H}), 3.25(\mathrm{~m}, 1 \mathrm{H}), 2.58-2.45(\mathrm{~m}, 3 \mathrm{H}), 2.44(\mathrm{ddd}, J=12.0,12.0,5.0,1 \mathrm{H}), 1.75-1.66(\mathrm{~m}, 2 \mathrm{H})$, $1.71(\mathrm{~s}, 3 \mathrm{H}), 1.69(\mathrm{~s}, 3 \mathrm{H}), 1.61-1.48(\mathrm{~m}, 4 \mathrm{H}), 1.41-1.22(\mathrm{~m}, 4 \mathrm{H}), 1.18(\mathrm{ddd}, J=13.5,7.5,5.5$, $1 \mathrm{H}), 0.94-0.91(\mathrm{~m}, 6 \mathrm{H}), 0.90(\mathrm{~s}, 9 \mathrm{H}), 0.87(\mathrm{~s}, 9 \mathrm{H}), 0.09(\mathrm{~s}, 6 \mathrm{H}), 0.06(\mathrm{~s}, 6 \mathrm{H}) ;{ }^{13} \mathrm{C}$ NMR $(125$ $\left.\mathrm{MHz}, \mathrm{CDCl}_{3}\right) \delta 170.6 .168 .7 .161 .5 .106 .7 .96 .0 .75 .9 .72 .0 .66 .4 .61 .3 . ~ 56.9 . ~ 42.6 .42 .2 .41 .9$. 40.5. 39.5. 37.2. 26.4. 26.2. 26.0. 25.9. 24.6. 20.1. 18.6. 18.5. 18.1. 14.2. 4.5, -4.9; LRMS (ESI): Mass calcd for $\mathrm{C}_{34} \mathrm{H}_{67} \mathrm{O}_{8} \mathrm{Si}_{2}[\mathrm{M}+\mathrm{H}]^{+}$, 660. Found $[\mathrm{M}+\mathrm{H}] 660[\alpha]_{\mathrm{D}}^{25}=+5.3\left(\mathrm{CHCl}_{3}, \mathrm{c}=0.5\right)$.

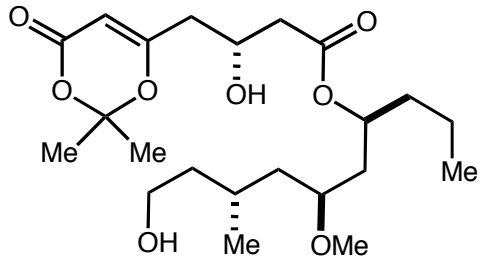

(R)-((4S,6S,8R)-10-hydroxy-6-methoxy-8-methyldecan-4-yl)-4-(2,2-dimethyl-4-oxo-4H-1,3dioxin-6-yl)-3-hydroxybutanoate (34): To a $0{ }^{\circ} \mathrm{C}$ solution of silyl ether $\mathbf{3 3}(81.5 \mathrm{mg}, 0.124$ $\mathrm{mmol})$ in THF $(2.5 \mathrm{~mL})$ was added $\mathrm{HF} \bullet$ pyridine $(0.22 \mathrm{~mL}, 2.48 \mathrm{mmol})$. The reaction mixture was allowed to warm to room temperature and was stirred for two hours. At this time, the reaction was then cooled to $0{ }^{\circ} \mathrm{C}$ and diluted with EtOAc $(2 \mathrm{~mL})$ and quenched with saturated aqueous $\mathrm{NaHCO}_{3}(10 \mathrm{~mL})$. The aqueous layer was separated and extracted with EtOAc $(5 \mathrm{X} 5$ $\mathrm{mL}$ ). The combined organic layers were dried with anhydrous $\mathrm{Na}_{2} \mathrm{SO}_{4}$, filtered and concentrated. The resulting residue was purified by flash column chromatography to afford 34 (49.7 mg, 93\%) as a clear oil. Analytical data for 34: IR (film) 3421, 2927, 1726, 1386, 1273, 1201, $1012 \mathrm{~cm}^{-1} ;{ }^{1} \mathrm{H}$ NMR (500 MHz, $\left.\mathrm{CDCl}_{3}\right) \delta 5.37(\mathrm{~s}, 1 \mathrm{H}), 5.20(\mathrm{~m}, 1 \mathrm{H}), 4.33(\mathrm{~m}, 1 \mathrm{H}), 4.032$ (bs, 1H), 3.37-3.31 (m, 1H), 3.33, (s, 3H), 2.57 (dd, $J=16.0,3.0,1 \mathrm{H}), 2.47$ (ddd, $J=20.5,9.0$, 9.0, 2H), 2.38 (dd, $J=14.5,4.5,1 \mathrm{H}), 2.053$ (bs, 1H), 1.85 (ddd, $J=13.5,10.0,2.5,1 \mathrm{H}), 1.75$ $1.49(\mathrm{~m}, 5 \mathrm{H}), 1.71(\mathrm{~s}, 6 \mathrm{H}), 1.43(\mathrm{ddd}, J=13.5,6.5,6.5,2 \mathrm{H}), 1.35-1.26(\mathrm{~m}, 2 \mathrm{H}), 1.14(\mathrm{ddd}, J=$ 13.0, 5.5, 5.5, 1H), 0.96-0.91 (m, 6H); $\left.{ }^{13} \mathrm{C} \mathrm{NMR} \mathrm{(125} \mathrm{MHz,} \mathrm{CDCl}_{3}\right) \delta 171.2,168.6,161.3,106.9$, 95.5, 76.4, 72.0, 65.1, 60.5, 56.9, 42.2, 40.8, 39.8, 38.3, 37.4, 26.0, 25.4, 25.0, 20.5, 18.7, 14.1; LRMS (ESI): Mass calcd for $\mathrm{C}_{22} \mathrm{H}_{39} \mathrm{O}_{8} \mathrm{Si}[\mathrm{M}+\mathrm{H}]^{+}, 431$. Found $[\mathrm{M}+\mathrm{H}]^{+} 431$. $[\alpha]_{\mathrm{D}}{ }^{25}=+2.8\left(\mathrm{CHCl}_{3}\right.$, $\mathrm{c}=1.0)$. 


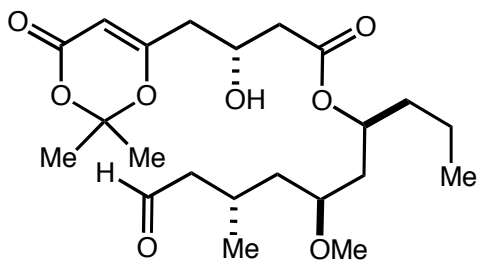

(R)-((4S,6S,8S)-6-methoxy-8-methyl-10-oxodecan-4-yl)-4-(2,2-dimethyl-4-oxo-4H-1,3-

dioxin-6-yl)-3-hydroxybutanoate (19): To a solution of diol 34 (49.7 $\mathrm{mg}, 0.116 \mathrm{mmol}$ ) in $\mathrm{CH}_{2} \mathrm{Cl}_{2}(1.9 \mathrm{~mL})$ was added TEMPO $(1.8 \mathrm{mg}, 0.012 \mathrm{mmol})$ and bisacetoxy iodobenzene (41.0 $\mathrm{mg}, 0.13 \mathrm{mmol})$. The reaction mixture stirred for four hours and was then diluted with $\mathrm{CH}_{2} \mathrm{Cl}_{2}(5$ $\mathrm{mL})$ and quenched with saturated $\mathrm{Na}_{2} \mathrm{~S}_{2} \mathrm{O}_{3}(20 \mathrm{~mL})$. The aqueous layer was separated and extracted with $\mathrm{CH}_{2} \mathrm{Cl}_{2}(5 \mathrm{X} 5 \mathrm{~mL})$. The combined organic layers were dried with anhydrous $\mathrm{Na}_{2} \mathrm{SO}_{4}$, filtered and concentrated to afford aldehyde $19(49.2 \mathrm{mg}, 99 \%)$ as a colorless oil. Aldehyde 19 was used directly in the next step. Analytical data for: ${ }^{1} \mathrm{H} \mathrm{NMR}\left(500 \mathrm{MHz}, \mathrm{CDCl}_{3}\right)$ $\delta 9.76(\mathrm{~s}, 1 \mathrm{H}), 5.37(\mathrm{~s}, 1 \mathrm{H}), 5.16(\mathrm{~m}, 1 \mathrm{H}), 4.33(\mathrm{~m}, 1 \mathrm{H}), 3.58(\mathrm{~m}, 1 \mathrm{H}), 3.38-3.26(\mathrm{~m}, 1 \mathrm{H}), 3.32$ (s, 3H), 2.57 (dd, $J=16.0,3.0,1 \mathrm{H}), 2.51-2.37(\mathrm{~m}, 2 \mathrm{H}), 2.32(\mathrm{dd}, J=7.5,2.0,1 \mathrm{H}), 2.29(\mathrm{dd}, J=$ 9.5, 4.5, 1H), $2.22(\mathrm{ddd}, J=13.5,13.5,7.0,1 \mathrm{H}), 1.82-1.74(\mathrm{~m}, 1 \mathrm{H}), 1.72(\mathrm{~s}, 3 \mathrm{H}), 1.71(\mathrm{~s}, 3 \mathrm{H})$, 1.67-1.65 (m, 2H), 1.64-1.56 (m, 1H), 1.55-1.48 (m, 2H), 1.38-1.23 (m, 3H), $1.02(\mathrm{~d}, J=6.5$, $3 \mathrm{H}), 0.93(\mathrm{dd}, J=7.5,7.5,3 \mathrm{H}) ;{ }^{13} \mathrm{C} \mathrm{NMR}\left(125 \mathrm{MHz}, \mathrm{CDCl}_{3}\right) \delta 202.7,171.6,168.5,161.2$, 106.9, 95.7, 76.2, 72.1, 65.1, 56.8, 51.6, 41.8, 41.2, 40.7, 38.4, 37.3, 29.9, 25.6, 24.9, 20.7, 18.8, 14.1; LRMS (ESI): Mass calcd for $\mathrm{C}_{22} \mathrm{H}_{36} \mathrm{O}_{8} \mathrm{Na}[\mathrm{M}+\mathrm{Na}]^{+}, 451$. Found [M+Na] ${ }^{+}, 451$.

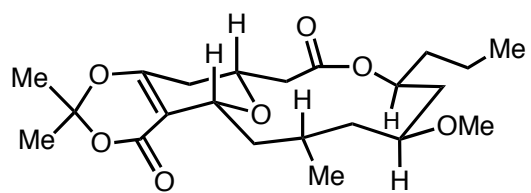

Tricyclic Dioxinone (35): To round bottom charged with aldehyde 19 (21.6 $\mathrm{mg}, 0.05 \mathrm{mmol}$ ), $\mathrm{CaSO}_{4}(20.6 \mathrm{mg}, 1.52 \mathrm{mmol})$, and $\mathrm{Sc}(\mathrm{OTf})_{3}(4.9 \mathrm{mg}, 0.01 \mathrm{mmol})$ was added $\mathrm{MeCN}(5.0 \mathrm{~mL})$. The reaction stirred for $45 \mathrm{~min}$ and then brine $(0.5 \mathrm{~mL})$ was added and the reaction mixture was filtered through a short pad of Celite eluting with $\mathrm{CH}_{2} \mathrm{Cl}_{2}$. The layers were separated and the aqueous layer was extracted with EtOAc $(4 \mathrm{X} 5 \mathrm{~mL})$. The combined organic layers were dried with anhydrous $\mathrm{Na}_{2} \mathrm{SO}_{4}$, filtered and concentrated. The resulting residue was purified by flash column chromatography (30\% EtOAc:hexanes) to afford tricyclic dioxinone $\mathbf{3 5}(5.0 \mathrm{mg}, \mathbf{2 5 \%}$ ) as a colorless oil. Analytical data for 35: IR (film) 2922, 1726, 1648, 1406, 1269, 1207, 1090, 999 $\mathrm{cm}^{-1} ;{ }^{1} \mathrm{H}$ NMR $\left(500 \mathrm{MHz}, \mathrm{CDCl}_{3}\right) \delta 5.25$ (dddd, $\left.J=9.0,4.0,4.0,4.0,1 \mathrm{H}\right), 4.44-4.42$ (d, $J=11.0$, $1 \mathrm{H}), 3.96$ (dddd, $J=12.5,9.0,5.0,2.0,1 \mathrm{H}), 3.34(\mathrm{~m}, 1 \mathrm{H}), 3.30(\mathrm{~s}, 3 \mathrm{H}), 2.91-2.84(\mathrm{~m}, 2 \mathrm{H}), 2.35$ $(\mathrm{dd}, J=12.5,2.5,1 \mathrm{H}), 2.15(\mathrm{~d}, J=18.5,1 \mathrm{H}), 2.00(\mathrm{~m}, 1 \mathrm{H}), 1.75-1.71(\mathrm{~m}, 1 \mathrm{H}), 1.70(\mathrm{~s}, 3 \mathrm{H}), 1.69$ $(\mathrm{s}, 3 \mathrm{H}), 1.63-1.49(\mathrm{~m}, 4 \mathrm{H}), 1.44(\mathrm{~m}, 1 \mathrm{H}), 1.40-1.32(\mathrm{~m}, 2 \mathrm{H}), 1.28-1.23(\mathrm{~m}, 2 \mathrm{H}), 1.06(\mathrm{~d}, J=7.5$, $3 \mathrm{H}), 0.93(\mathrm{dd}, J=7.5,7.5,3 \mathrm{H}) ;{ }^{13} \mathrm{C} \mathrm{NMR}\left(125 \mathrm{MHz}, \mathrm{CDCl}_{3}\right) \delta 170.6,163.2,159.6,106.3$, 105.1, 76.9, 73.6, 72.3, 68.4, 56.2, 43.5, 42.3, 42.1, 39.8, 37.6, 32.1, 27.7, 27.2, 23.7, 22.5, 18.9, 14.1 ;LRMS (ESI): Mass calcd for $\mathrm{C}_{44} \mathrm{H}_{68} \mathrm{O}_{14} \mathrm{Na}[2 \mathrm{M}+\mathrm{Na}]^{+}, 843$. Found $[2 \mathrm{M}+\mathrm{Na}]^{+}, 843 .[\alpha]_{\mathrm{D}}{ }^{25}=$ $+198.6\left(\mathrm{CHCl}_{3}, \mathrm{c}=0.9\right)$. 


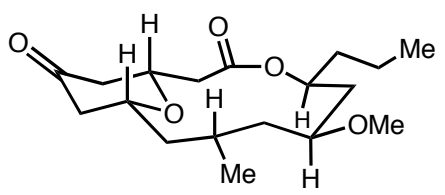

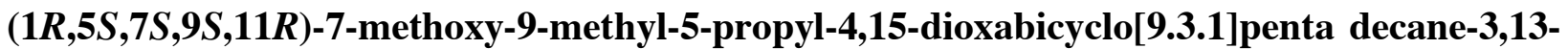
dione (20): To a one-dram vial charged with tricyclic dioxinone 35 (5.0 $\mathrm{mg}, 0.01 \mathrm{mmol})$ was added DMSO $(0.25 \mathrm{~mL})$ and $\mathrm{H}_{2} \mathrm{O}(0.6 \mathrm{~mL})$. The reaction was then submerged into a preheated oil bath of $130{ }^{\circ} \mathrm{C}$. The reaction stirred for $12 \mathrm{hrs}$ and was then diluted with EtOAc $(2 \mathrm{~mL})$ and added to a separatory funnel containing brine $(125 \mathrm{~mL})$. The layers were separated and the aqueous layer was extracted with EtOAc $(5 \mathrm{X} 5 \mathrm{~mL})$. The combined organic layers were dried with anhydrous $\mathrm{Na}_{2} \mathrm{SO}_{4}$, filtered and concentrated. The resulting residue was purified by flash column chromatography (30\% EtOAc:hexanes) to afford pyranone $20(3.0 \mathrm{mg}, 82 \%)$ as a colorless oil. Analytical data for 20: IR (film) 2958, 2920, 1725, 1651, 1558, 1273, 1249, 1091 $\mathrm{cm}^{-1}$; ${ }^{1} \mathrm{H}$ NMR $\left(500 \mathrm{MHz}, \mathrm{CDCl}_{3}\right) \delta 5.25-5.20(\mathrm{~m}, 1 \mathrm{H}), 4.05$ (apt, $\left.J=10.2,1 \mathrm{H}\right), 3.59$ (apt, $J=$ 9.72, 1H), 3.51 (apt, $J=9.7,1 \mathrm{H}), 3.344(\mathrm{~s}, 3 \mathrm{H}), 2.72(\mathrm{dd}, J=15.1,4.34,1 \mathrm{H}), 2.53$ (dd, $J=14.6$, $10.7,1 \mathrm{H}), 2.44(\mathrm{~d}, J=14.1,1 \mathrm{H}), 2.35-2.22(\mathrm{~m}, 2 \mathrm{H}), 1.86(\mathrm{apt}, J=12.2,1 \mathrm{H}), 1.73-1.60(\mathrm{~m}, 3 \mathrm{H})$, $1.55-1.45$ (m, 2H), 1.43-1.35 (m, 4H), 1.20 (apt, $J=12.1,2 \mathrm{H}), 1.01$ (d, $J=6.8,3 \mathrm{H}), 0.93$ (dd, $J$ $=7.3,3 \mathrm{H}) ;{ }^{13} \mathrm{C} \mathrm{NMR}\left(125 \mathrm{MHz}, \mathrm{CDCl}_{3}\right) \delta 206.1,170.2,79.2,77.5,75.9,73.5,56.5,49.0,47.2$, 44.4, 42.7, 40.2, 37.1, 31.3, 25.7, 19.1, 14.1; LRMS (ESI): Mass calcd for $\mathrm{C}_{36} \mathrm{H}_{62} \mathrm{O}_{11}\left[2 \mathrm{M}+\mathrm{H}_{2} \mathrm{O}\right]^{+}$, 670. Found $\left[2 \mathrm{M}+\mathrm{H}_{2} \mathrm{O}\right]^{+}, 670 .[\alpha]_{\mathrm{D}}^{25}=+32.6\left(\mathrm{CHCl}_{3}, \mathrm{c}=0.1\right)$.

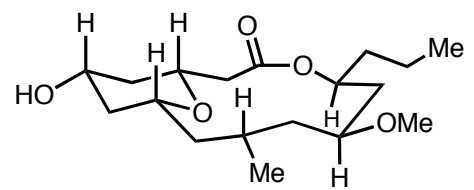

Alcohol 36: To a one dram vial charged with pyranone $20(3.0 \mathrm{mg}, 0.01 \mathrm{mmol})$ was added $\mathrm{MeOH}(0.2 \mathrm{~mL})$. The reaction mixture was cooled to $0{ }^{\circ} \mathrm{C}$ and $\mathrm{NaBH}_{4}(0.5 \mathrm{mg}, 0.02 \mathrm{mmol})$ was added. After $10 \mathrm{~min}$, AcOH $(7.0 \mu \mathrm{L})$ was added and the reaction was concentrated in vacuo. The resulting residue was purified by flash column chromatography (50\% EtOAc:hexanes) to afford pyran 36 (2.9 mg, 96\%) as a colorless oil. Analytical data for 36: IR (film) 3416, 2918,

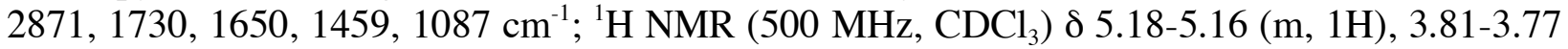
$(\mathrm{m}, 1 \mathrm{H}), 3.75$ (apt, $J=11.2,1 \mathrm{H}), 3.68(\mathrm{apt}, J=10.2,1 \mathrm{H}), 3.32(\mathrm{~s}, 3 \mathrm{H}), 3.18$ (apt, $J=10.2,1 \mathrm{H})$, $2.63(\mathrm{dd}, J=14.6,3.9,1 \mathrm{H}), 2.43(\mathrm{dd}, J=14.1,11.2,1 \mathrm{H}), 1.98(\mathrm{apd}, J=12.2,1 \mathrm{H}), 1.86(\mathrm{apt}, J=$ $11.2,2 \mathrm{H}$ ), 1.70 (dddd, $J=5.8,5.8,5.8,5.8,1 \mathrm{H}), 1.59$ (apt, $J=12.7,2 \mathrm{H}), 1.51-1.50$ (m, 3H), $1.50-1.42(\mathrm{~m}, 1 \mathrm{H}), 1.37-1.33(\mathrm{~m}, 4 \mathrm{H}), 1.30-1.12(\mathrm{~m}, 2 \mathrm{H}), 0.99(\mathrm{~d}, J=6.353 \mathrm{H}), 0.92(\mathrm{t}, J=7.32$, $3 \mathrm{H}) ;{ }^{13} \mathrm{C}$ NMR $\left(125 \mathrm{MHz}, \mathrm{CDCl}_{3}\right) \delta 171.0,78.8,75.7,73.4,72.5,68.3,56.5,44.3,42.5,42.4$, 42.1, 40.9, 40.2, 37.1, 31.4, 25.8, 19.2, 14.1; LRMS (ESI): Mass calcd for $\mathrm{C}_{36} \mathrm{H}_{64} \mathrm{O}_{10} \mathrm{Na}$ $[2 \mathrm{M}+\mathrm{Na}]^{+}, 328.44$. Found $[2 \mathrm{M}+\mathrm{Na}]^{+}, 679.9 .[\alpha]_{\mathrm{D}}{ }^{25}=+18.1\left(\mathrm{CHCl}_{3}, \mathrm{c}=0.1\right)$. 


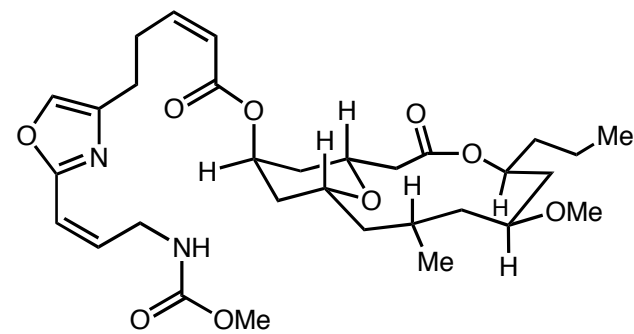

Neopeltolide (21): To a one dram vial charged with pyran 36 ( $2.9 \mathrm{mg}, 0.009 \mathrm{mmol})$, oxazole 3 ( $8.6 \mathrm{mg}, 0.032 \mathrm{mmol})$ and $\mathrm{PPh}_{3}(6.84 \mathrm{mg}, 0.027 \mathrm{mmol})$ was added benzene $(0.36 \mathrm{~mL})$. To the reaction mixture was added diisopropyl azodicarboxylate $(5.2 \mu \mathrm{L}, 0.027 \mathrm{mmol})$. After five min the reaction was concentrated in vacuo. The resulting residue was purified by flash column chromatography (30\% EtOAc:hexanes) to afford $21(4.2 \mathrm{mg}, 79 \%)$ as a colorless oil. Analytical data for 21: IR (film) 3356, 3132, 2921, 2497, 1718, 1645, 1458, 1394, 1273, 1167, 1084, 1026, $777 \mathrm{~cm}^{-1} ;{ }^{1} \mathrm{H}$ NMR $\left(600 \mathrm{MHz}, \mathrm{CD}_{3} \mathrm{OD}\right) \delta 7.67$ (s, 1H), $6.40(\mathrm{ddd}, J=11.5,7.4,7.4,1 \mathrm{H}), 6.29$ (ddd, $J=11.9,4.2,2.1,1 \mathrm{H}), 6.05$ (ddd, $J=11.9,6.0,6.0,1 \mathrm{H}), 5.90$ (ddd, $J=13.1,3.3,1.6,1 \mathrm{H}$ ), $5.22-5.21(\mathrm{~m}, 1 \mathrm{H}), 5.19-5.16(\mathrm{~m}, 1 \mathrm{H}), 4.32(\mathrm{~d}, J=4.94,2 \mathrm{H}), 4.09$ (dddd, $J=11.3,11.3,4.2$, 2.1, $1 \mathrm{H}), 3.68(\mathrm{dd}, J=9.5,9.5,1 \mathrm{H}), 3.66(\mathrm{~s}, 3 \mathrm{H}), 3.56(\mathrm{t}, J=9.88,1 \mathrm{H}), 3.29(\mathrm{~s}, 3 \mathrm{H}), 3.03$ (ddd, $J$ $=7.5,7.5,7.5,2 \mathrm{H}), 2.74(\mathrm{dd}, J=7.3,7.3,2 \mathrm{H}), 2.72(\mathrm{dd}, J=10.8,3.7,1 \mathrm{H}), 2.32(\mathrm{dd}, J=14.7$, $10.8,1 \mathrm{H}), 1.89$ (ddd, $J=14.2,10.8,1 \mathrm{H}), 1.85-1.83(\mathrm{~m}, 1 \mathrm{H}), 1.75-1.71(\mathrm{~m}, 1 \mathrm{H}), 1.71-1.68(\mathrm{~m}$, $1 \mathrm{H}), 1.61-1.50(\mathrm{~m}, 4 \mathrm{H}), 1.44-1.29(\mathrm{~m}, 7 \mathrm{H}), 1.12(\mathrm{ddd}, J=12.9,10.9,1.9,1 \mathrm{H}), 1.00(\mathrm{~d}, J=6.7$, $3 \mathrm{H}), 0.96(\mathrm{dd}, J=14.8,7.4,3 \mathrm{H}) ;{ }^{13} \mathrm{C}$ NMR $\left(125 \mathrm{MHz}, \mathrm{CD}_{3} \mathrm{OD}\right) \delta 173.2,166.9,162.0,159.0$, $150.1,142.4,139.3,136.0,121.8,116.0,77.2,77.1,74.0,71.4,69.3,56.5,52.7,45.3,43.6,43.3$, 41.1, 38.0, 37.5, 36.3, 32.7, 29.1, 26.5, 26.1, 20.1, 14.2; HRMS (ESI): Exact mass calcd for $\mathrm{C}_{31} \mathrm{H}_{47} \mathrm{~N}_{2} \mathrm{O}_{9}[\mathrm{M}+\mathrm{H}]^{+}$, 591.3282. Found $\left.[\mathrm{M}+\mathrm{H}]^{+}, 591.3277[\alpha]_{\mathrm{D}}{ }^{25}=+23.8\left(\mathrm{CH}_{3} \mathrm{OH}\right), \mathrm{c}=0.24\right)$. Reported $[\alpha]_{\mathrm{D}}^{25}$ for natural neopeltolide $\left.=+24\left(\mathrm{CH}_{3} \mathrm{OH}\right), \mathrm{c}=0.24\right){ }^{7}$

7. Wright, A. E.; Botelho, J. C.; Guzman, E.; Harmody, D.; Linley, P.; McCarthy, P. J.; Pitts, T. P.; Pomponi, S. A.; Reed, J. K. J. Nat. Prod. 2007, 70, 412-416. 
Comparison of ${ }^{13} \mathrm{C}$ Spectral Data of Neopeltolide, 1 , and 21

\section{neopeltolide ${ }^{7}$ isolated}

14.1

20.0

26.0

26.4

29.0

32.6

36.2

37.4

37.9

41.0

41.0

43.2

43.5

45.2

52.6

56.4

69.2

71.3

73.9

77.0

77.1

115.7

121.7

135.9

139.2

142.3

150.0

159.6

161.9

166.9

173.0

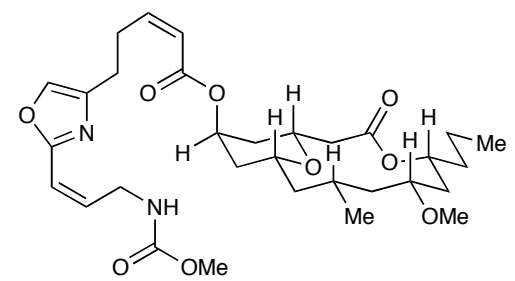

Synthetic 1

(proposed structure)

12.9

18.4

23.9

25.1

27.7

34.3

34.8

35.8

37.5

39.7

40.0

41.9

44.3

44.8

51.3

55.3

67.7

71.5

74.4

75.2

83.2

114.7

120.4

134.7

137.9

141.0

148.8

158.4

160.6

165.6

173.4

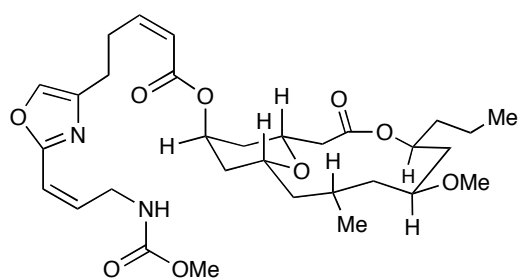

Synthetic 21

(actual structure)
14.2

20.1

26.1

26.5

29.1

32.7

36.3

37.5

38.0

41.1

41.1

43.3

43.6

45.3

52.7

56.5

69.3

71.4

74.0

77.1

77.2

116.2

121.8

136.0

139.3

142.4

150.1

159.0

162.0

166.9

173.2 


\section{Comparison of ${ }^{1} \mathbf{H}$ Spectral Data of Natural Neopeltolide and Synthetic Neopeltolide}

Isolated neopeltolide

$0.92, \mathrm{t}(7.6)$

0.94 , d (6.9)

$1.08, \mathrm{~m}$

$1.25, \mathrm{~m}$

$1.28, \mathrm{~m}$

1.33, $\mathrm{m}$

1.36, $\mathrm{m}$

$1.38, \mathrm{~m}$

$1.46, \mathrm{~m}$

$1.48, \mathrm{~m}$

$1.49, \mathrm{~m}$

$1.54, \mathrm{~m}$

$1.64, \mathrm{~m}$

1.68, m

$1.78, \mathrm{~m}$

1.83, $\mathrm{m}$

2.26 , dd $(15.1,11.0)$

2.66 , dd $(15.1,4.1)$

2.68 , dd $(7.6,7.6)$

2.98, $\mathrm{m}$

3.23, s

3.55 , bt (10.3)

$3.62, \mathrm{~s}$

$3.64, \mathrm{~m}$

4.04, ddt $(4.1,2.1)$

4.28 , bd (4.8)

5.14 , dt $(4.8,9.6)$

$5.17, \mathrm{~m}$

5.86, dt $(11.7,1.4)$

6.02, dt $(11.7,6.2)$

6.24, dt $(11.7,6.2)$

6.33, dt $(11.7,7.6)$

$7.64, \mathrm{~s}$
Synthetic neopeltolide (21)

$$
\begin{gathered}
0.96, \text { dd }(7.4,7.4) \\
1.00, d(6.7)
\end{gathered}
$$

1.12 , ddd $(12.9,10.9,1.9)$

$1.25, \mathrm{~m}$

1.29, $\mathrm{m}$

1.29-1.44, m

1.33, $\mathrm{m}$

1.36, $\mathrm{m}$

1.38, $\mathrm{m}$

$1.48, \mathrm{~m}$

1.50-1.61, m

$1.54, \mathrm{~m}$

1.68-1.71, m

$1.73, \mathrm{~m}$

1.84, $\mathrm{m}$

1.89 , ddd $(14.2,10.8)$

2.32 , dd $(14.7,10.8)$

2.72, dd $(10.8,3.7)$

2.74 , dd $(7.3,7.3)$

3.03 , ddd $(7.5,7.5,7.5)$

$3.29, \mathrm{~s}$

3.56 , dd $(9.8,9.8)$

$3.66(\mathrm{~s})$

3.68 , dd $(9.5,9.5)$

4.09 , dddd $(11.3,11.3,4.2,2.1)$

$4.32, d(4.9)$

$5.16, \mathrm{~m}$

$5.21, \mathrm{~m}$

5.90, ddd $(13.1,3.3,1.6)$

6.05 , ddd $(11.9,6.0,6.0)$

6.29 , ddd $(11.9,4.2,2.1)$

6.40 , ddd $(11.5,7.4,7.4)$

$7.67, \mathrm{~s}$ 


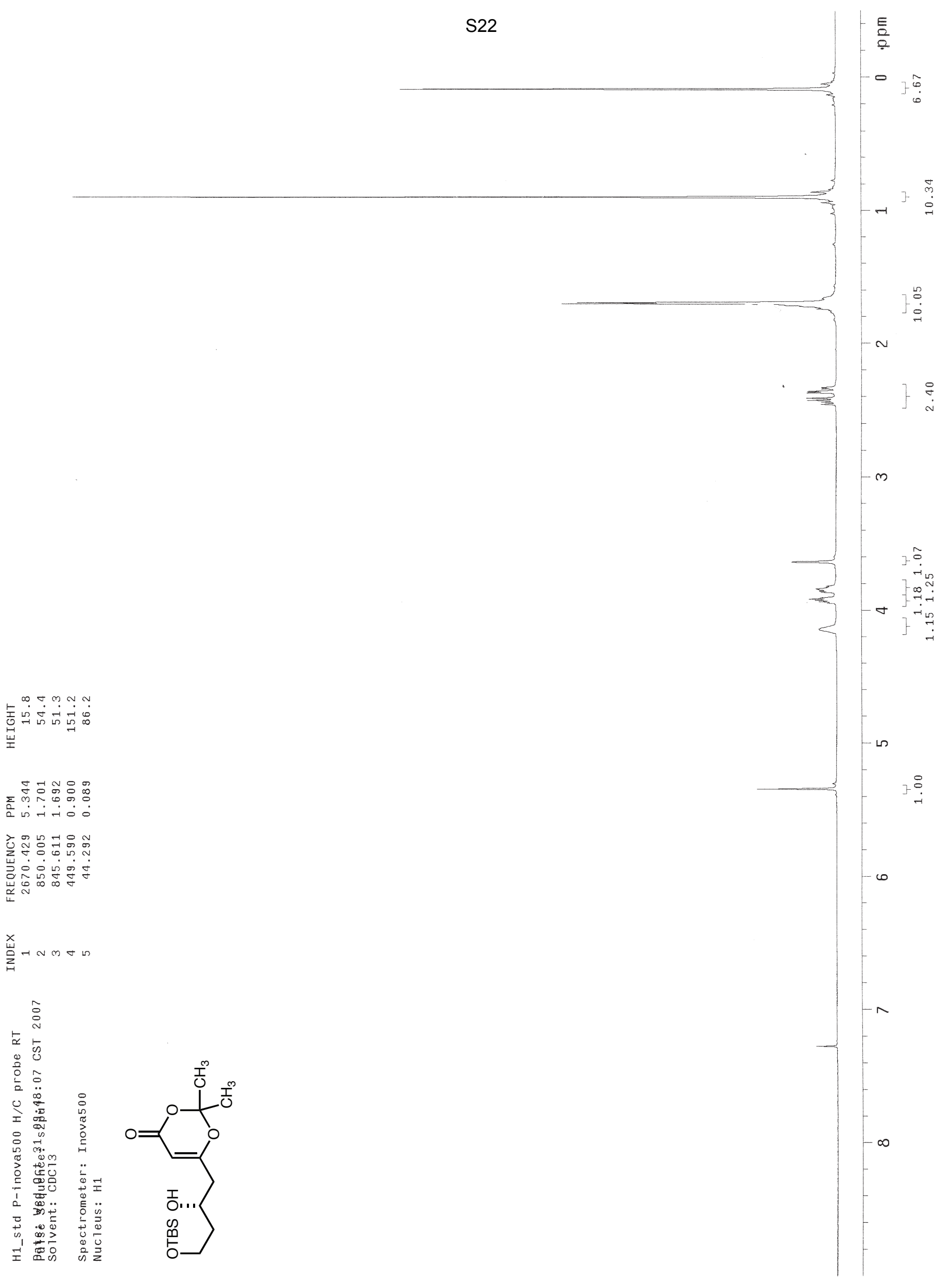


농

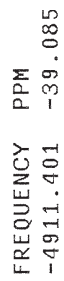

总-

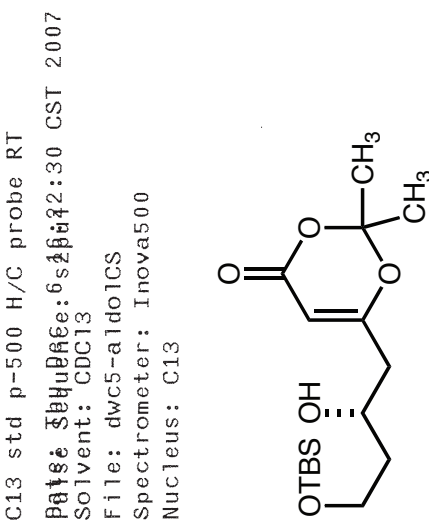




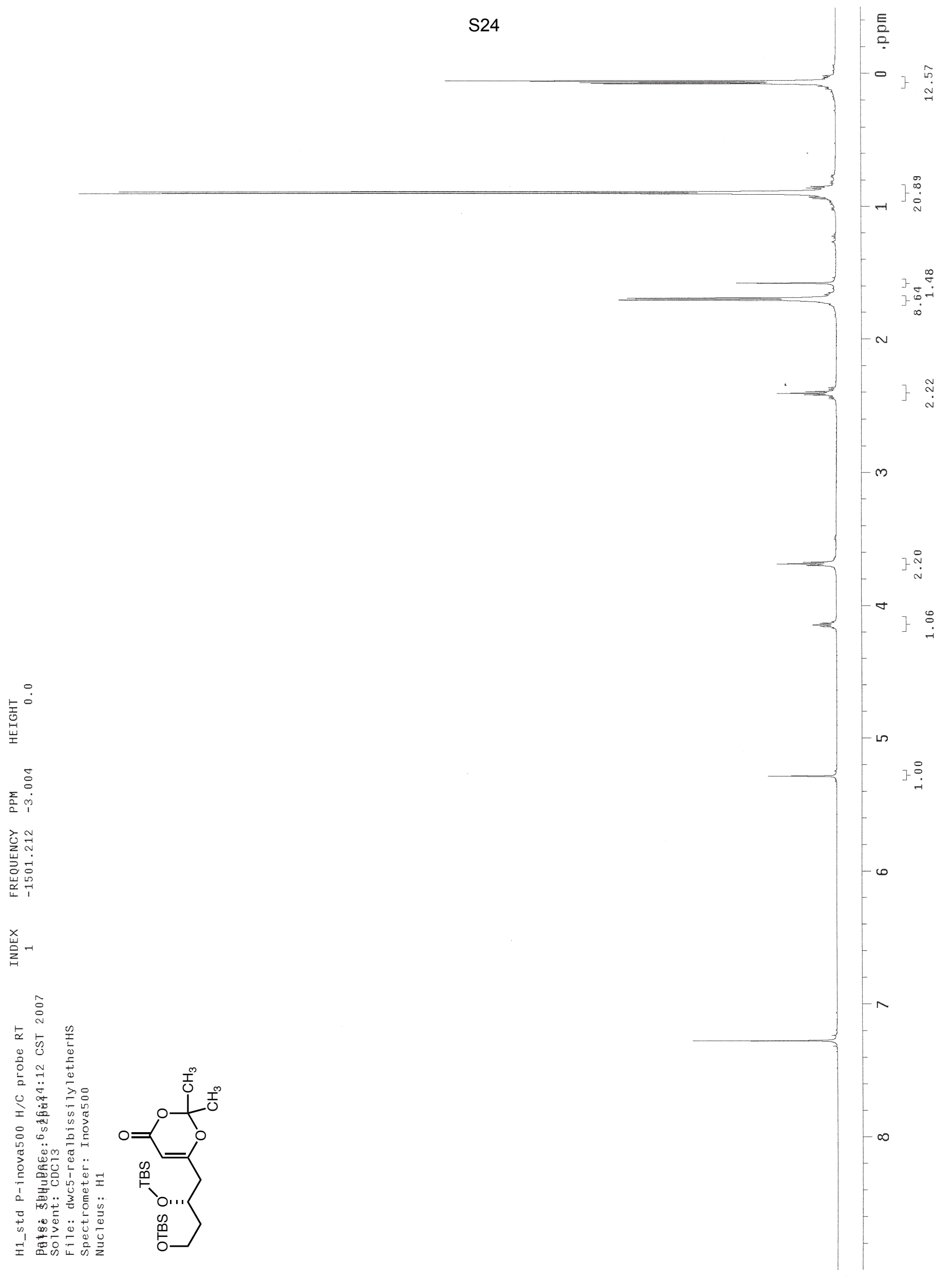




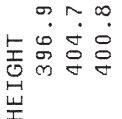

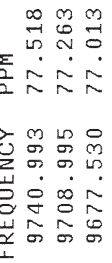

$\sum_{\text {岁 }}^{x} \sim m$

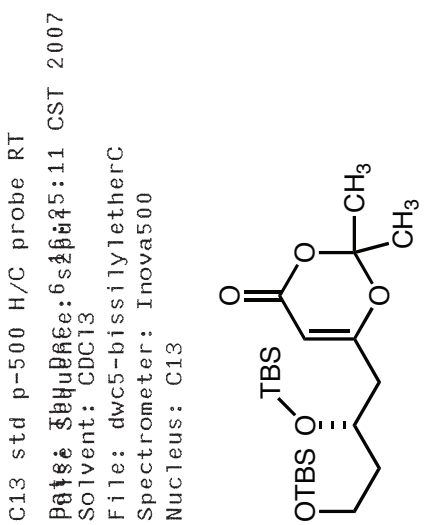




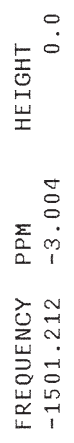
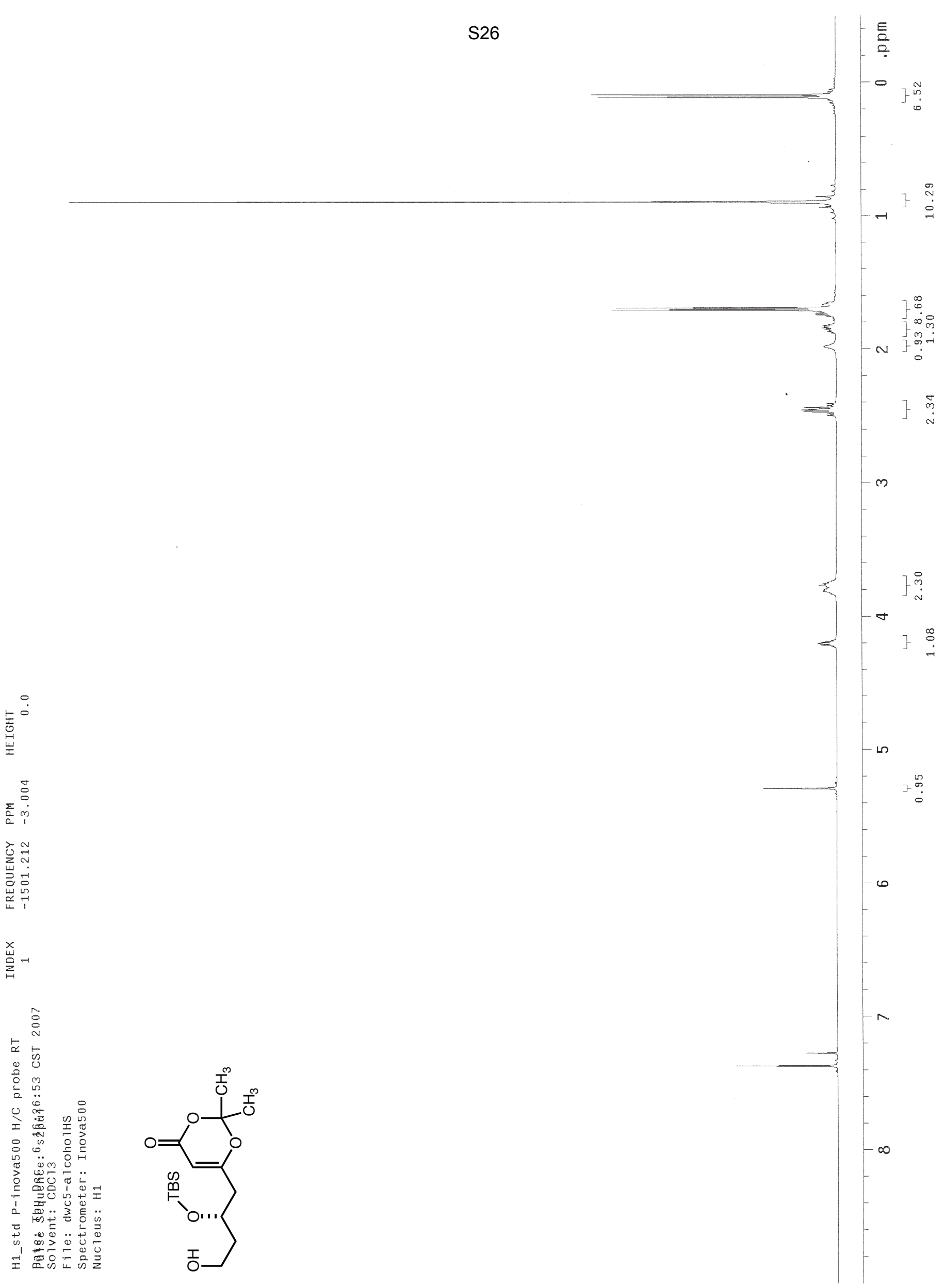


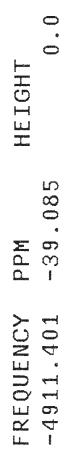

慈-

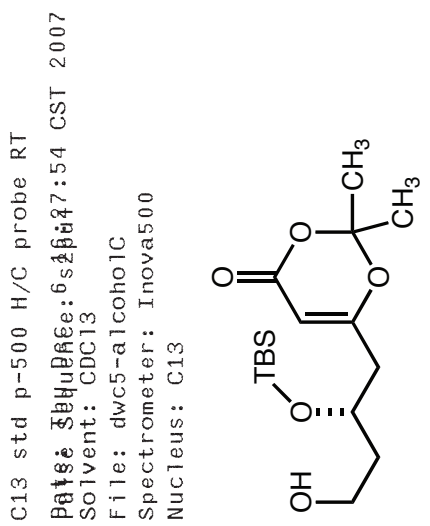




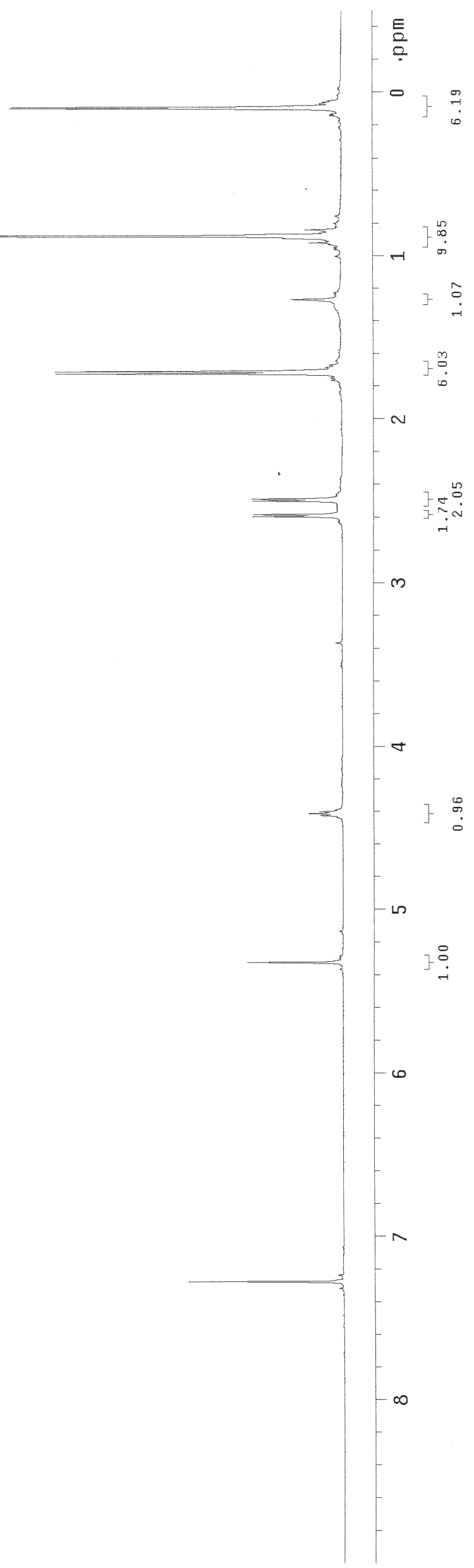




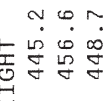

嵀

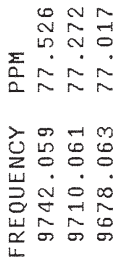

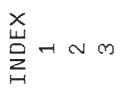

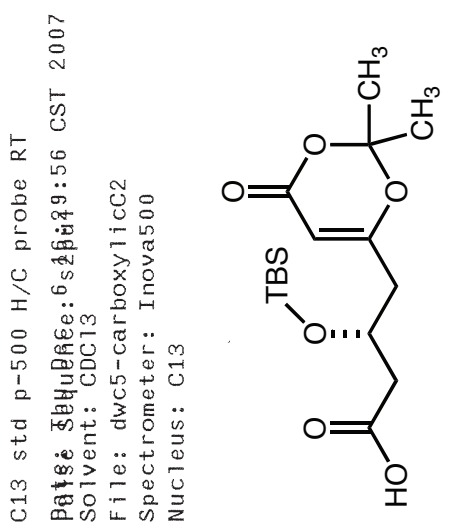


党

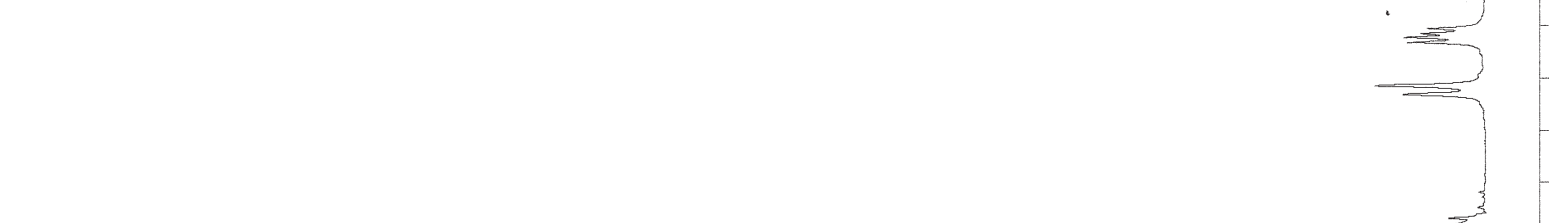

$+1 \stackrel{0}{-1}$

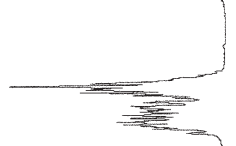

름

ट্른

岁

炭?

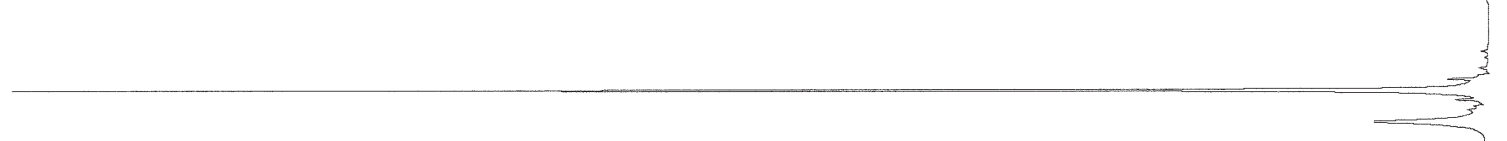

离-

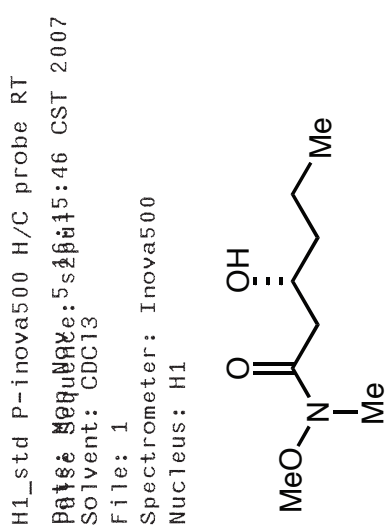


S31

농

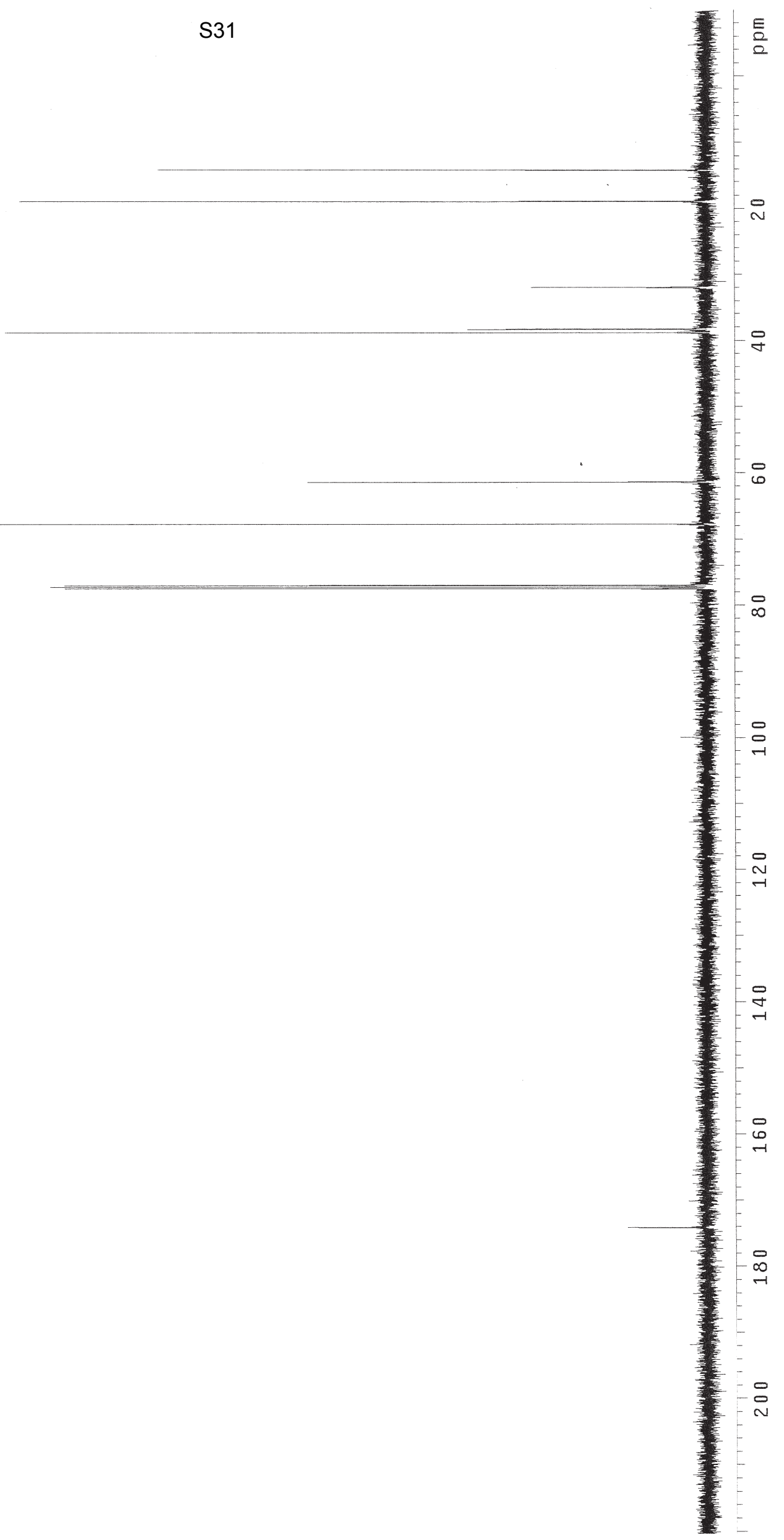




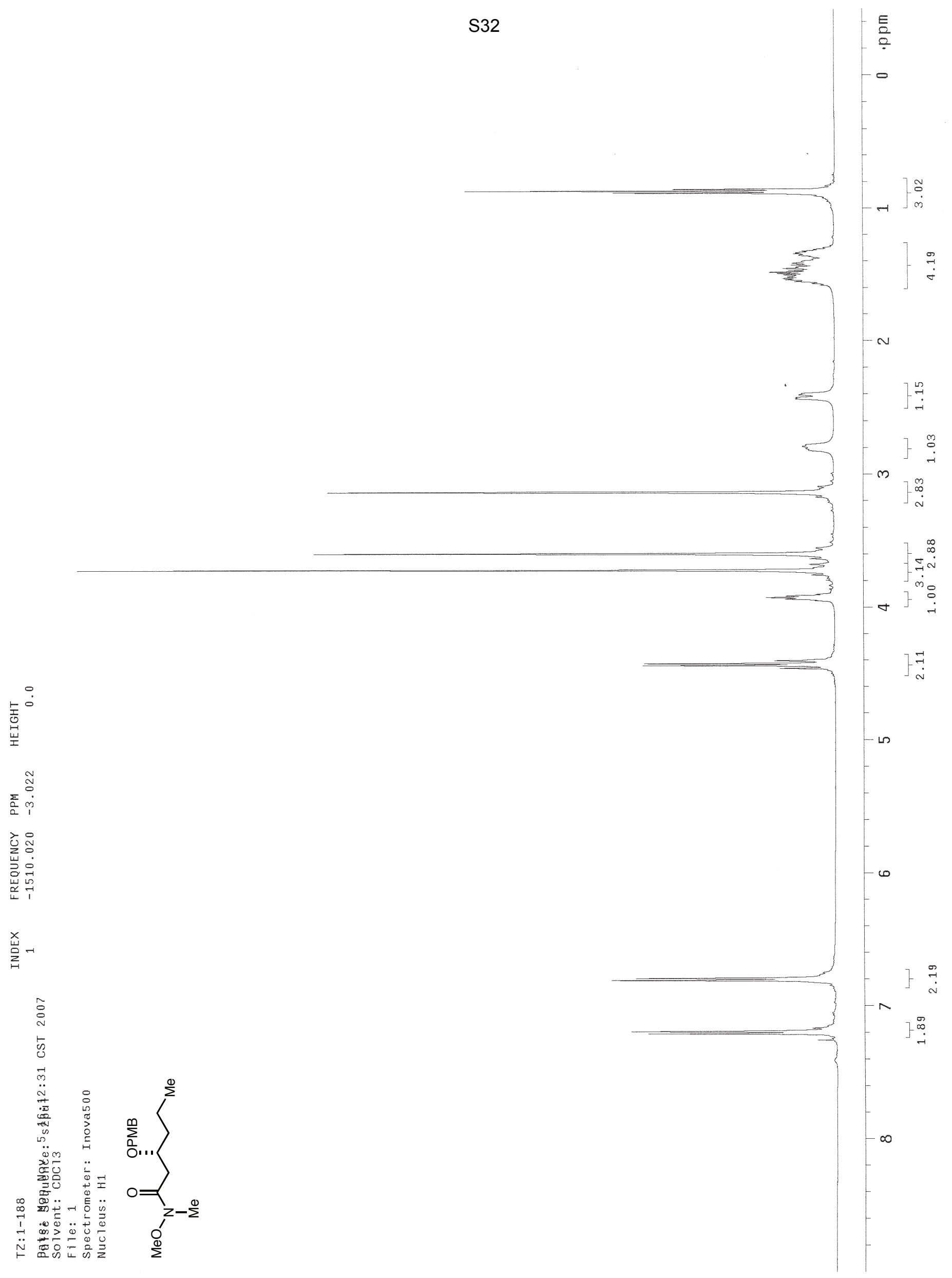




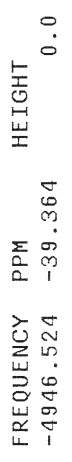

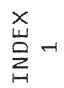

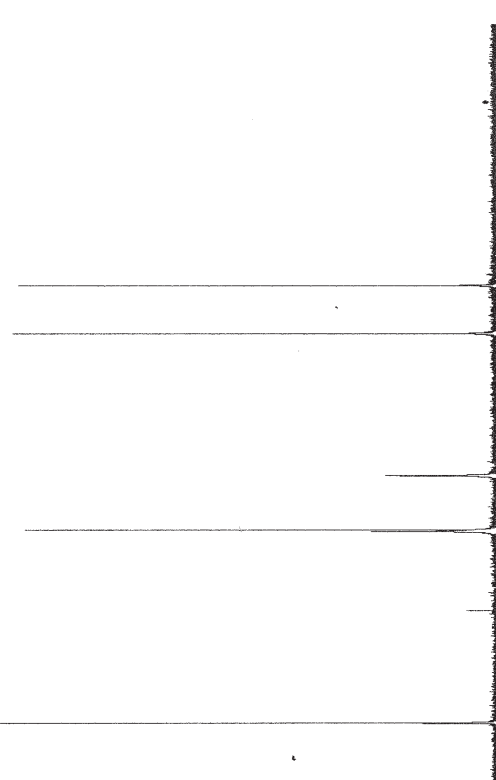

틀

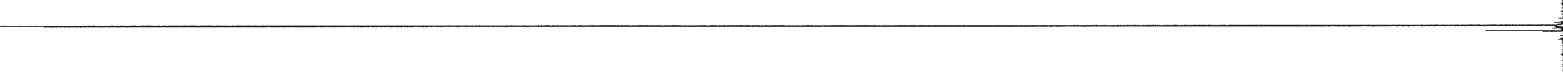

0
-1
-1

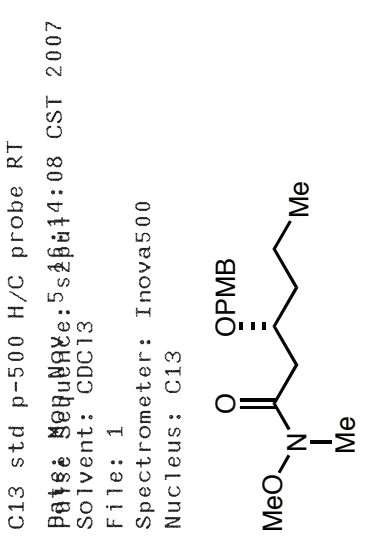




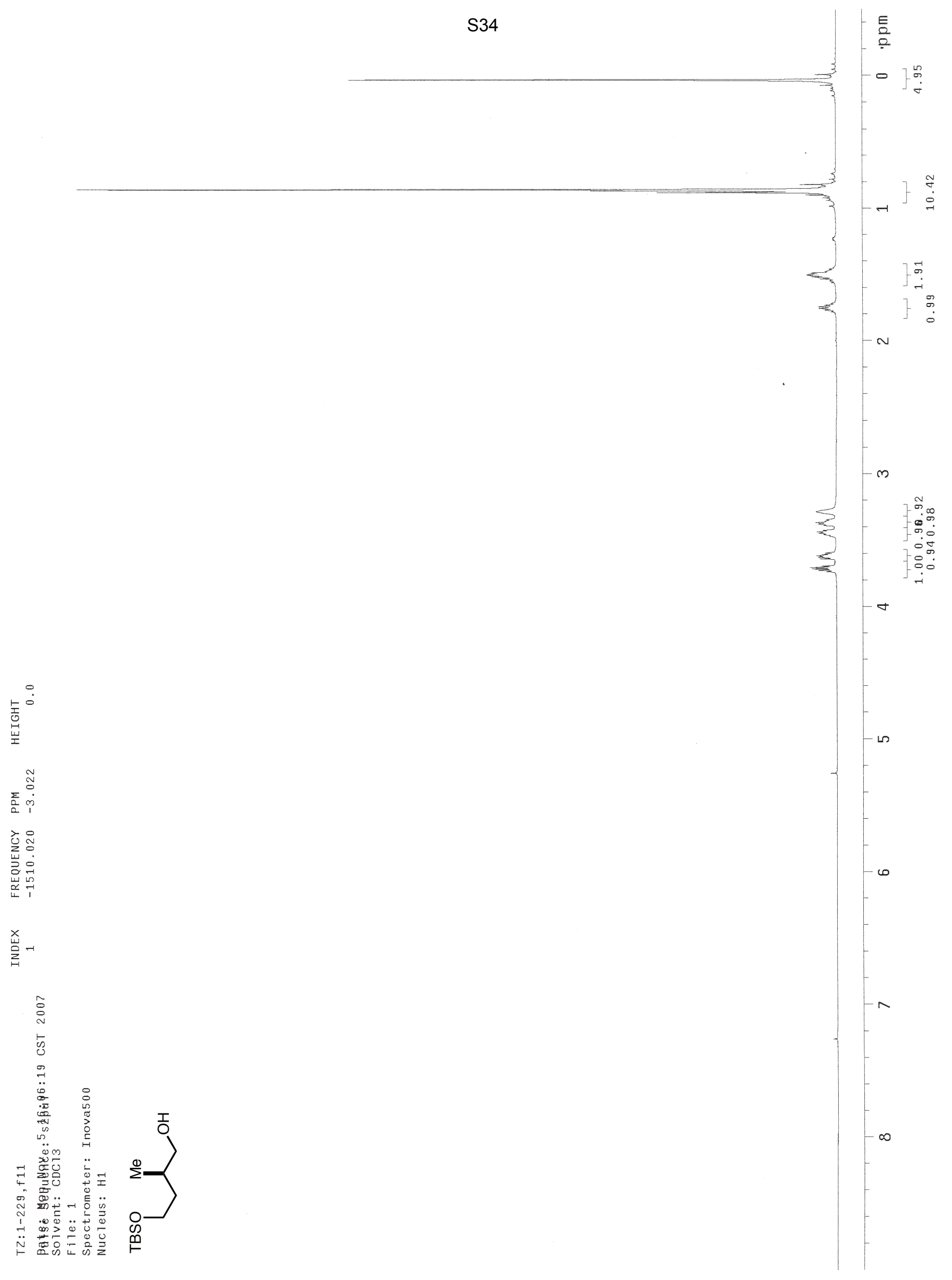




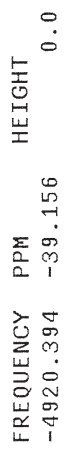

总-
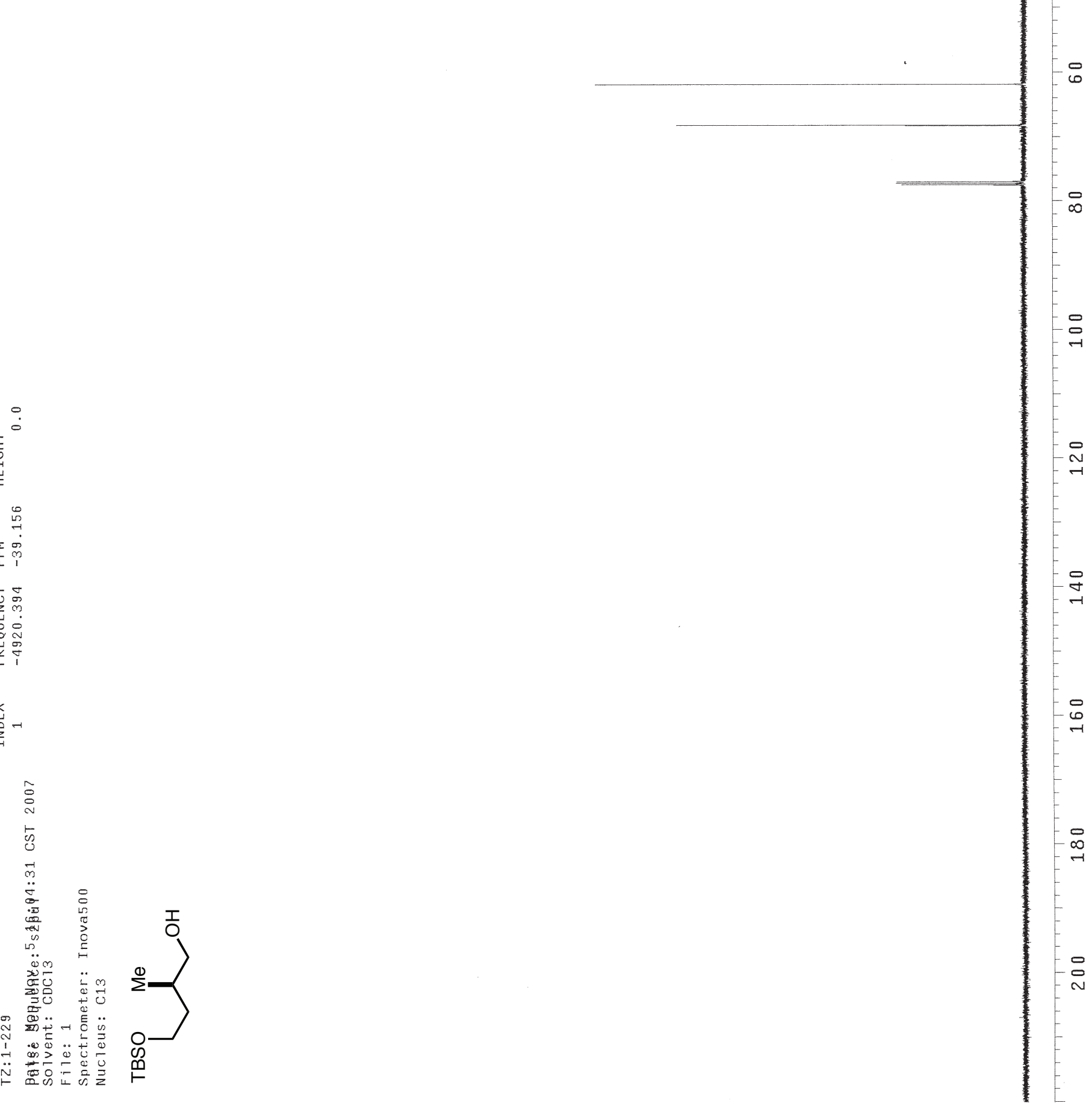


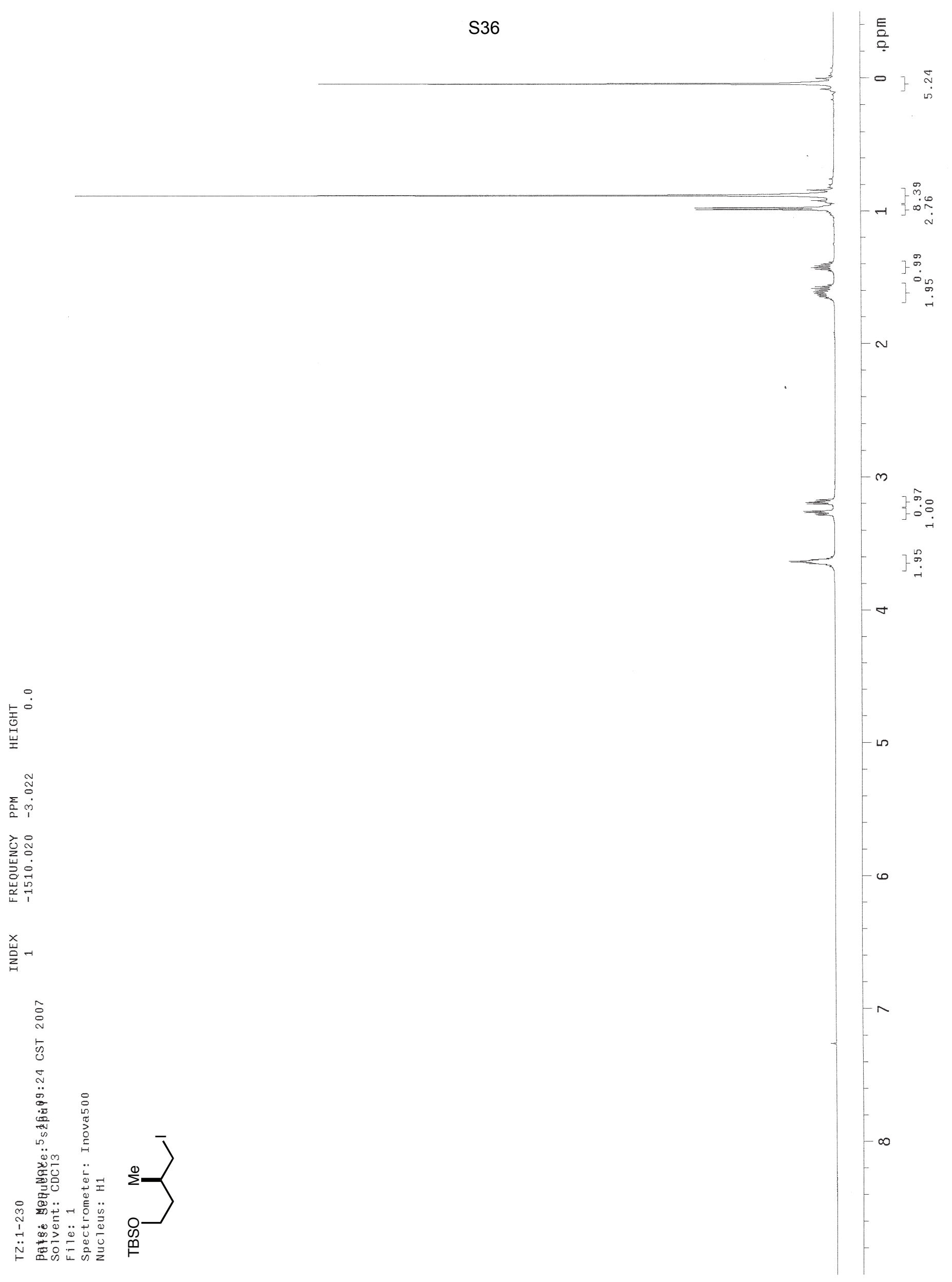




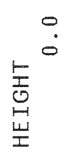

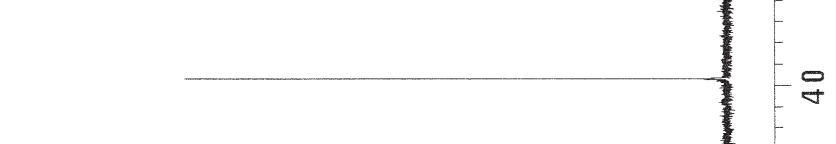

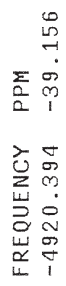

$\sum_{i=1}^{x}$

$\underset{-1}{\infty}$

å

5

幽

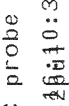

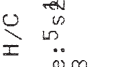

近

1 워

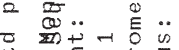

순

$m \geq 00$

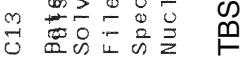




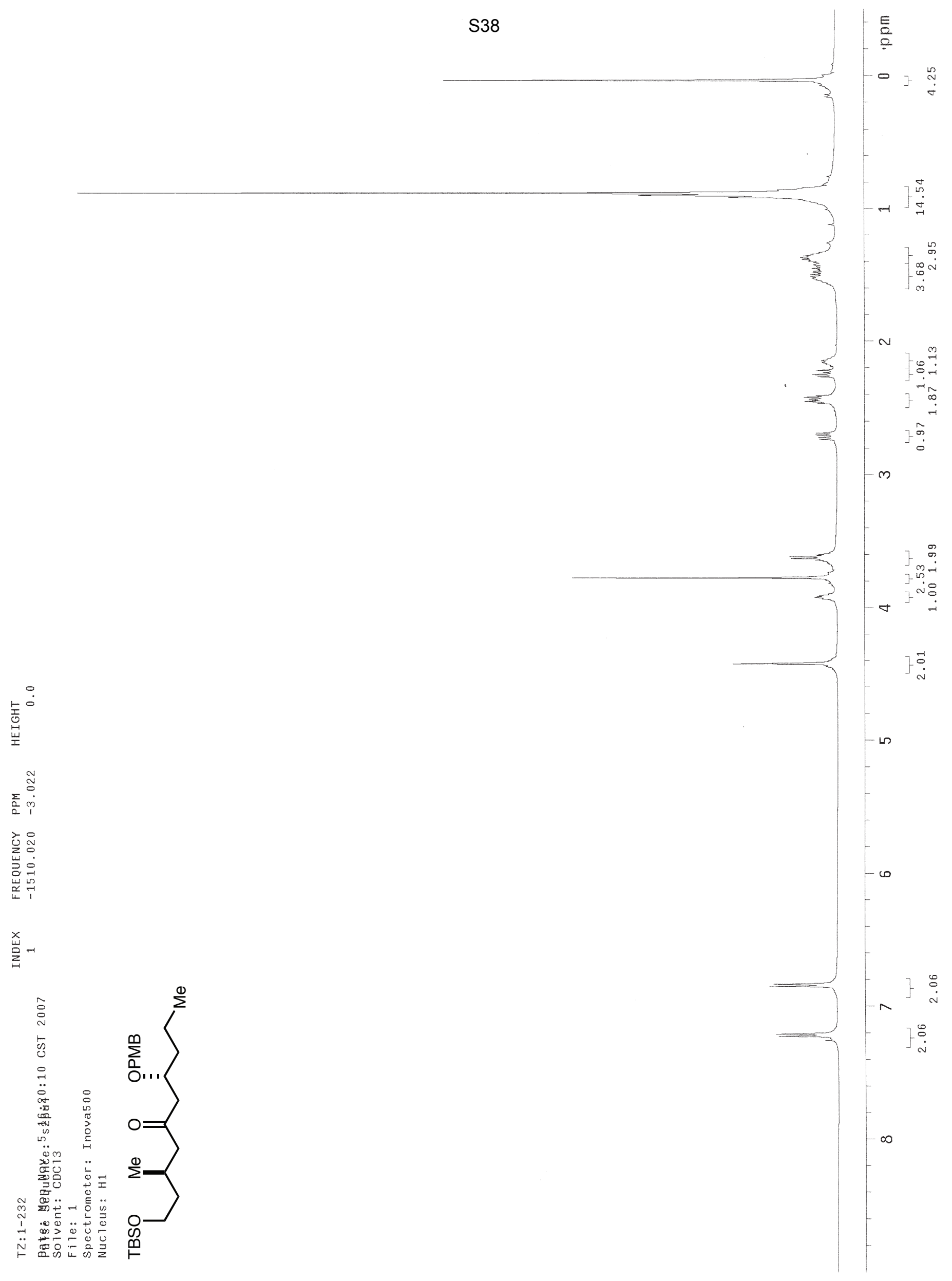


S39

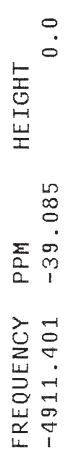

㞾-
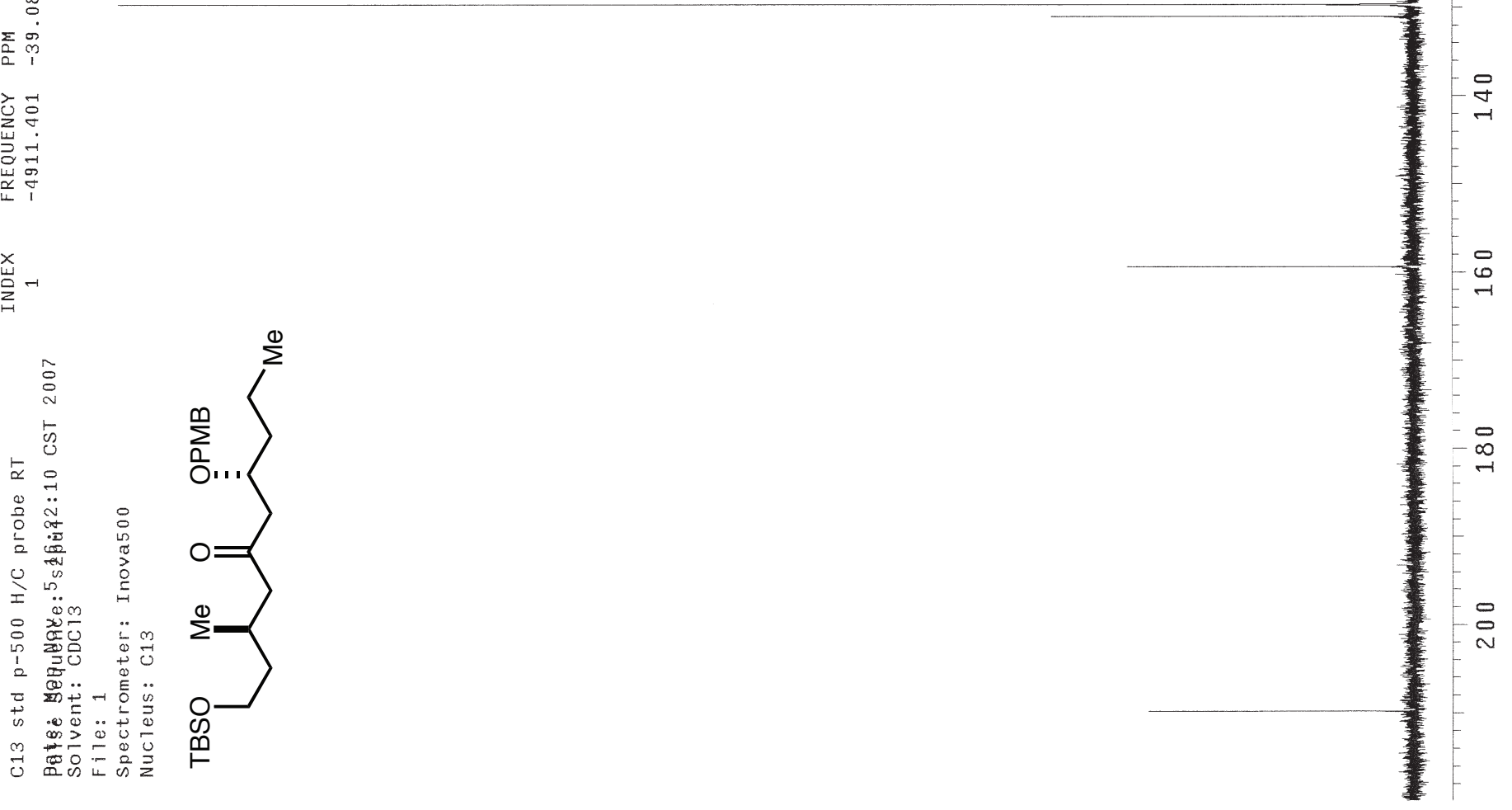


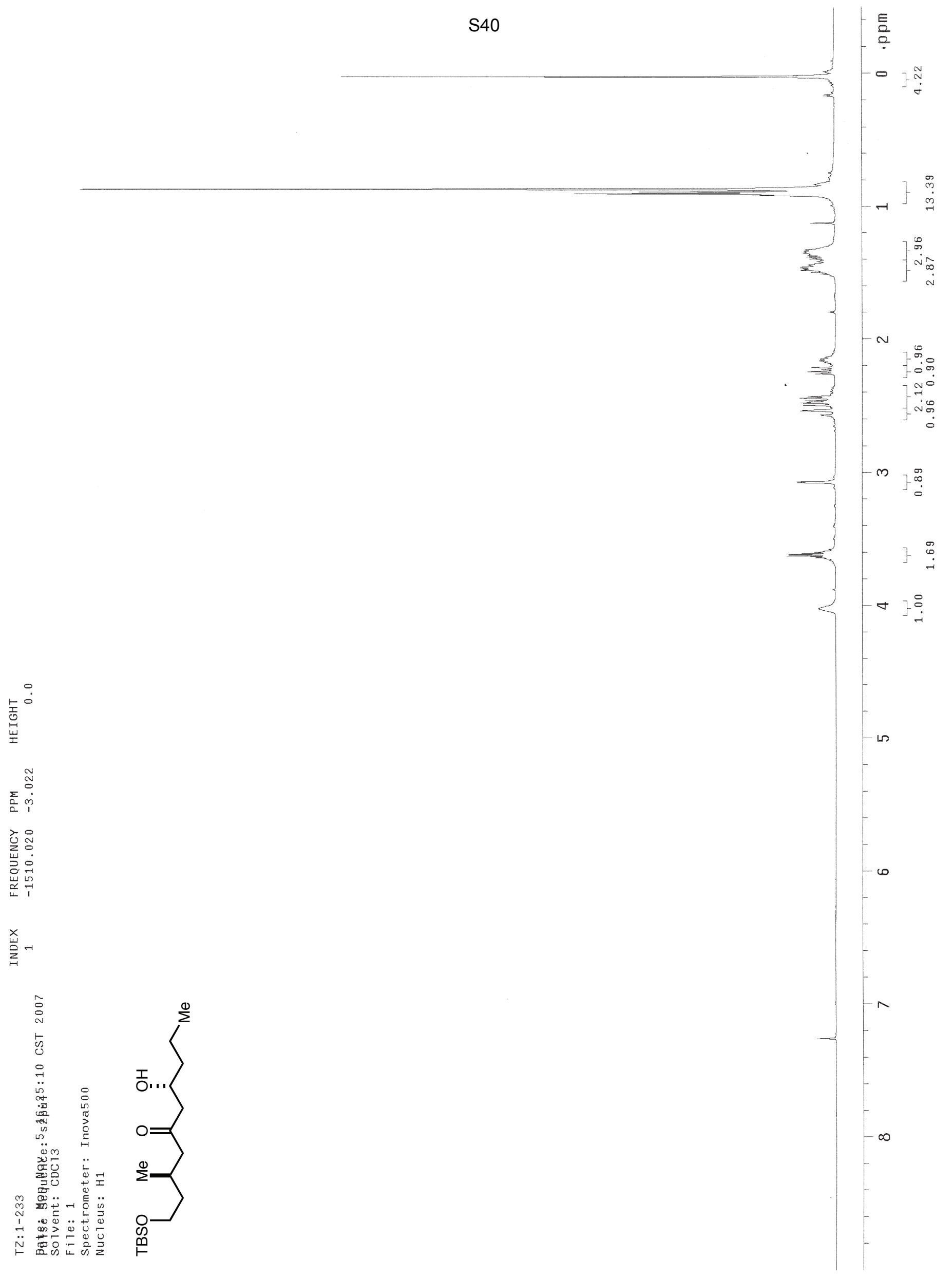




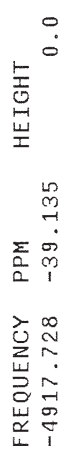

㞾-
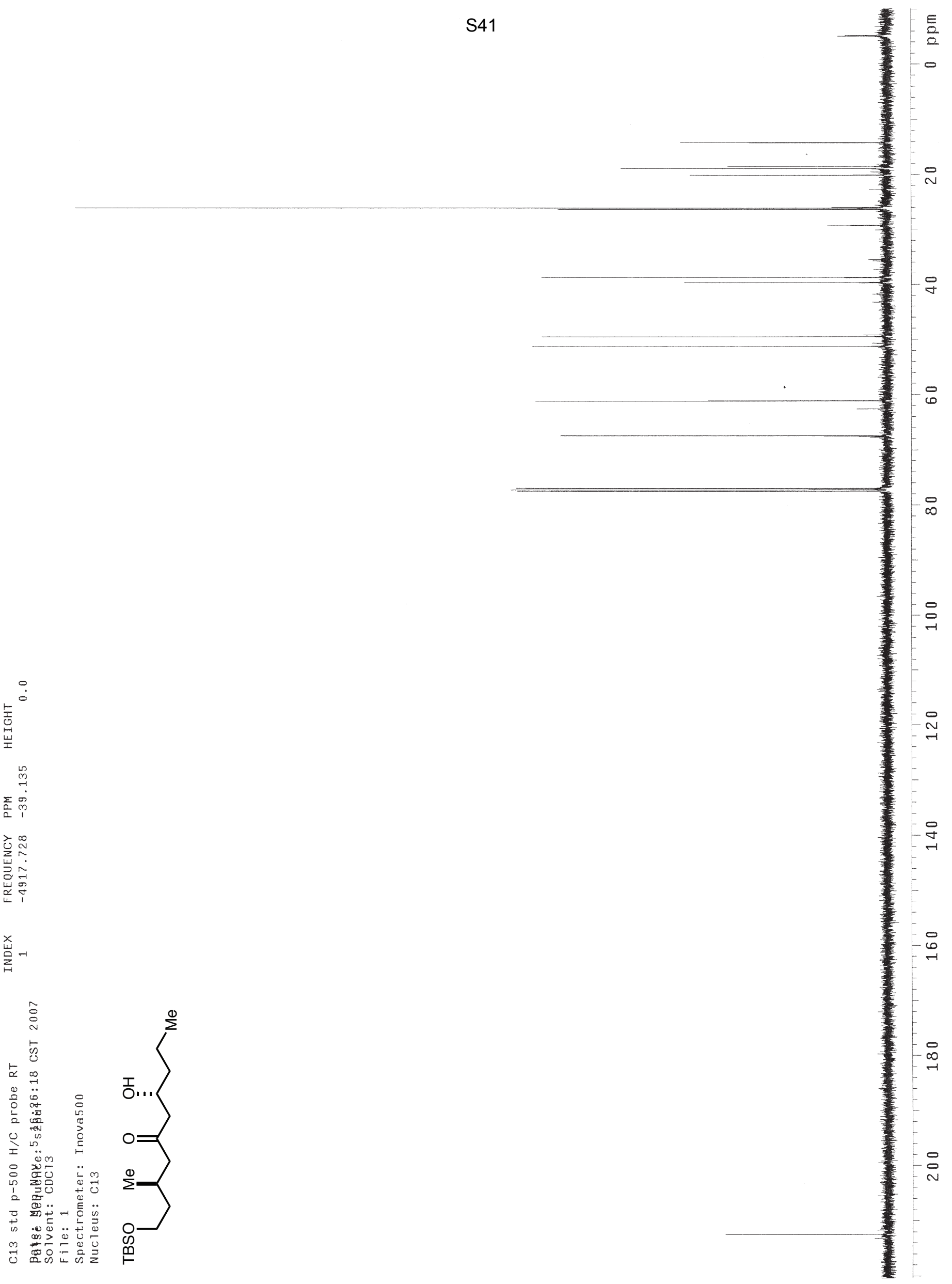


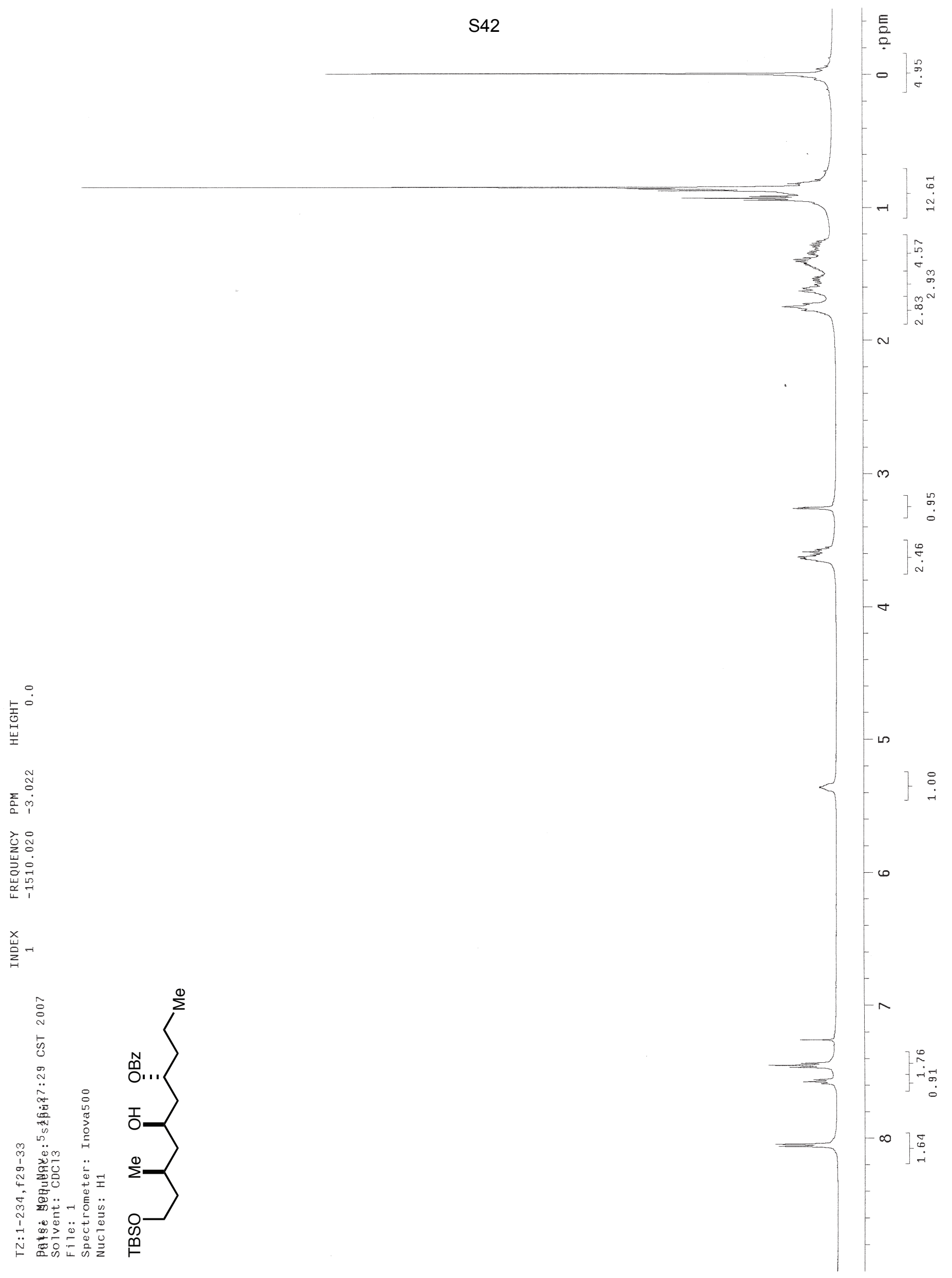





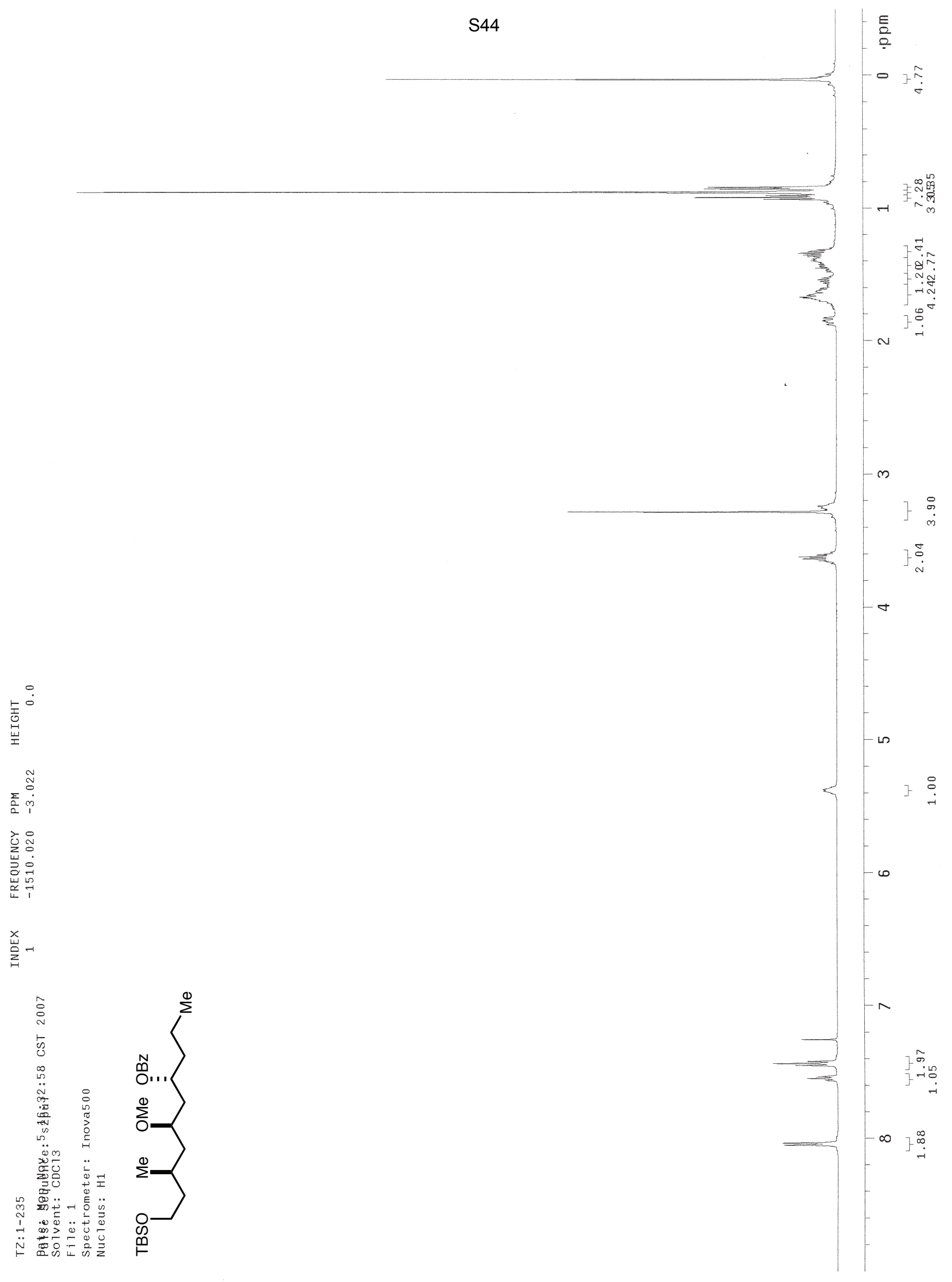


몽

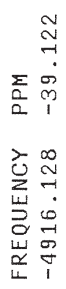

$\sum_{\substack{u \\ \hdashline}}^{x}$

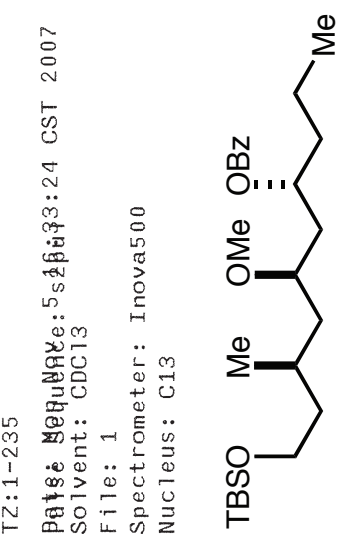

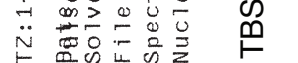


동

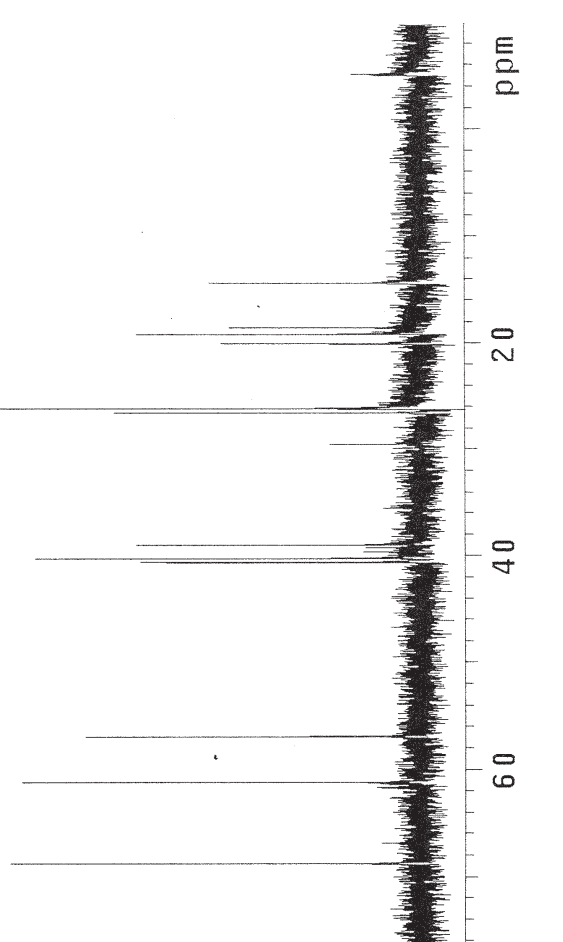

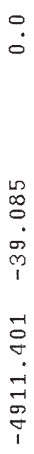

岩-1

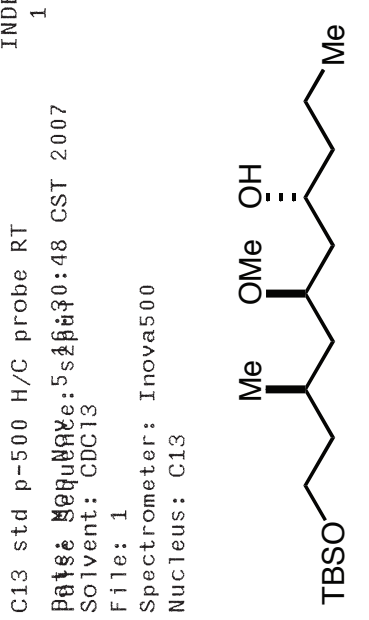




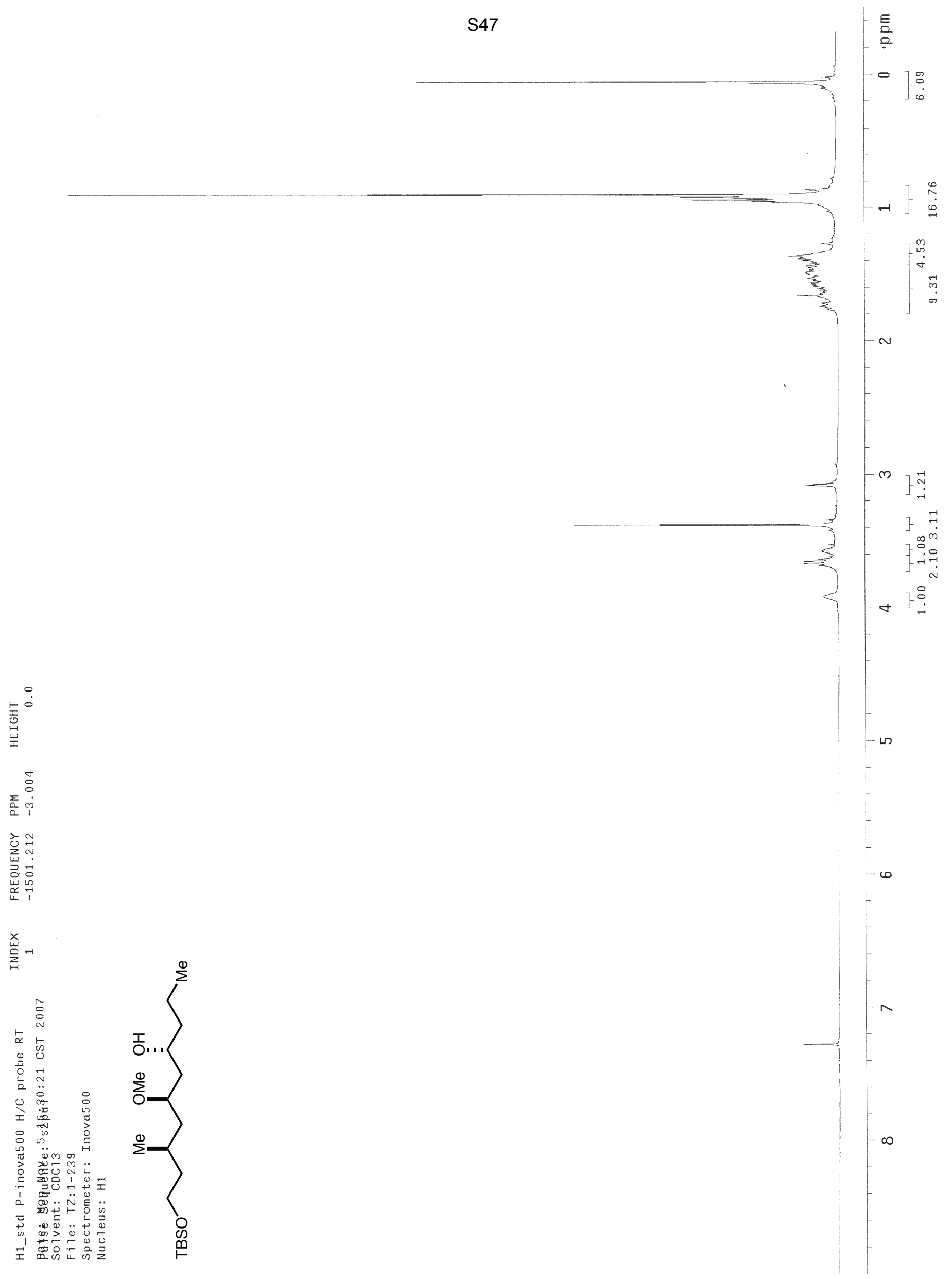




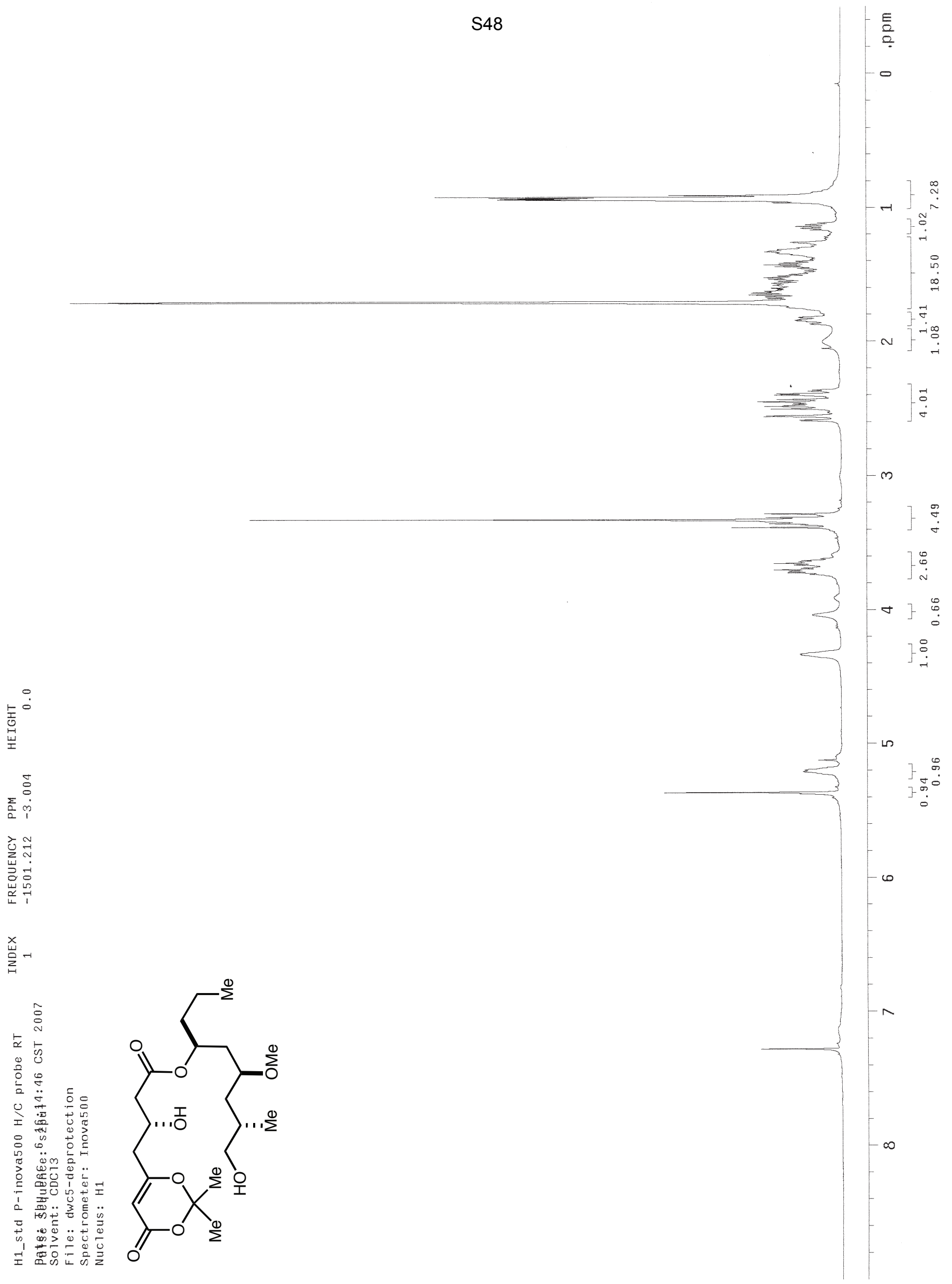




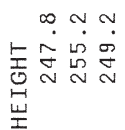

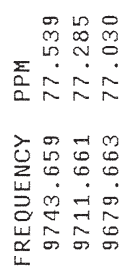

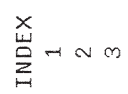
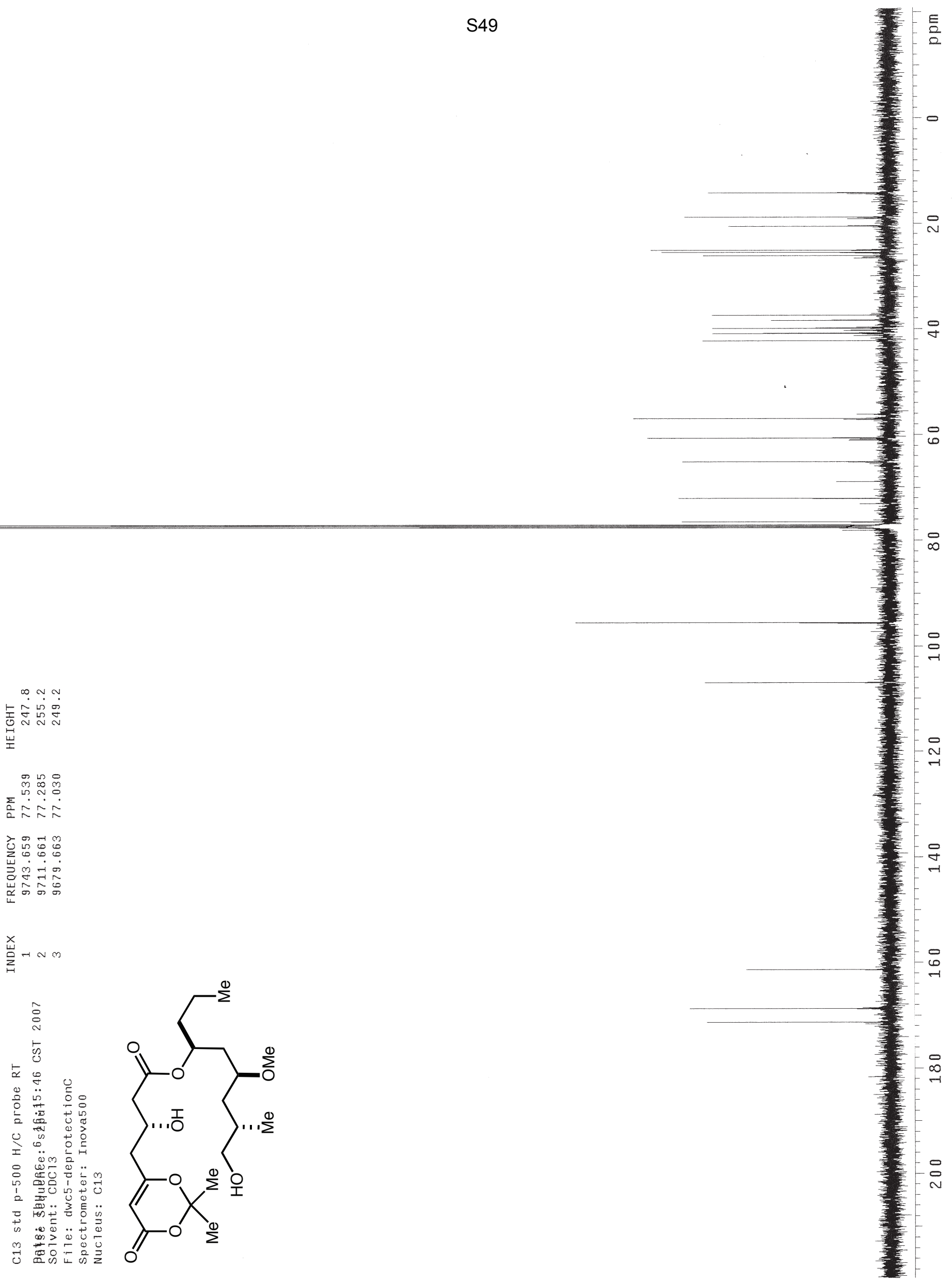


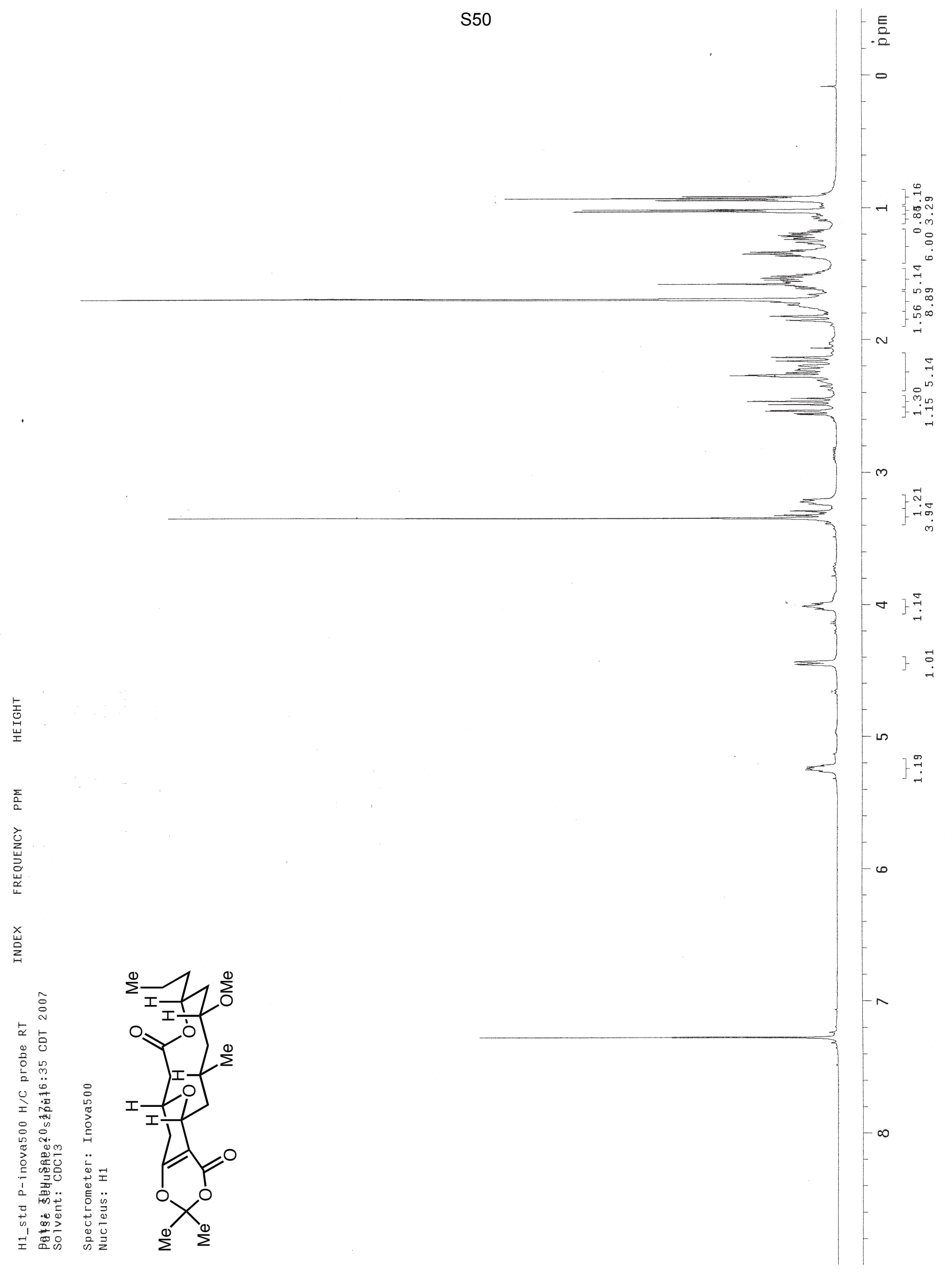




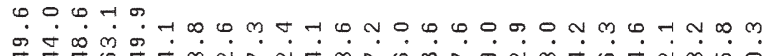
志施

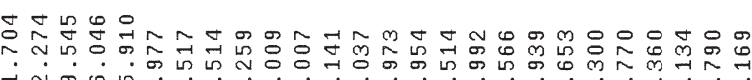

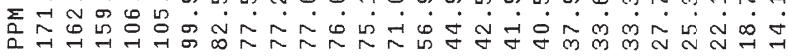

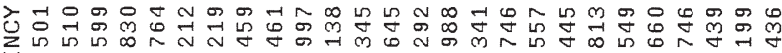

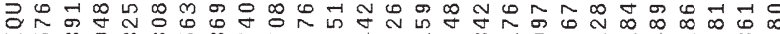

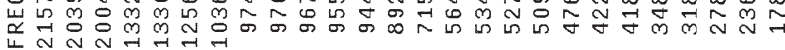

宸

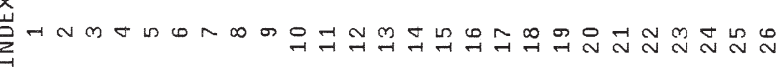
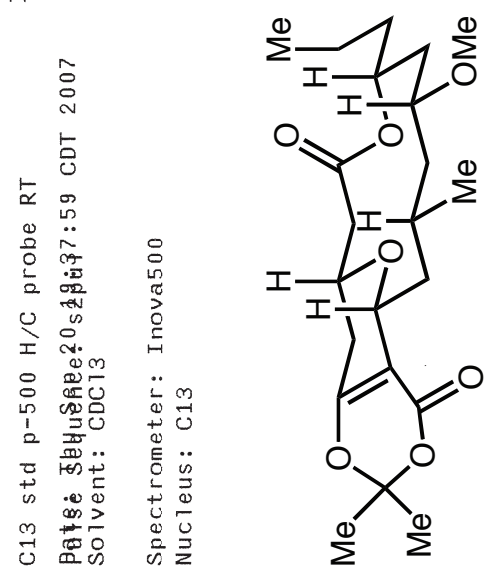


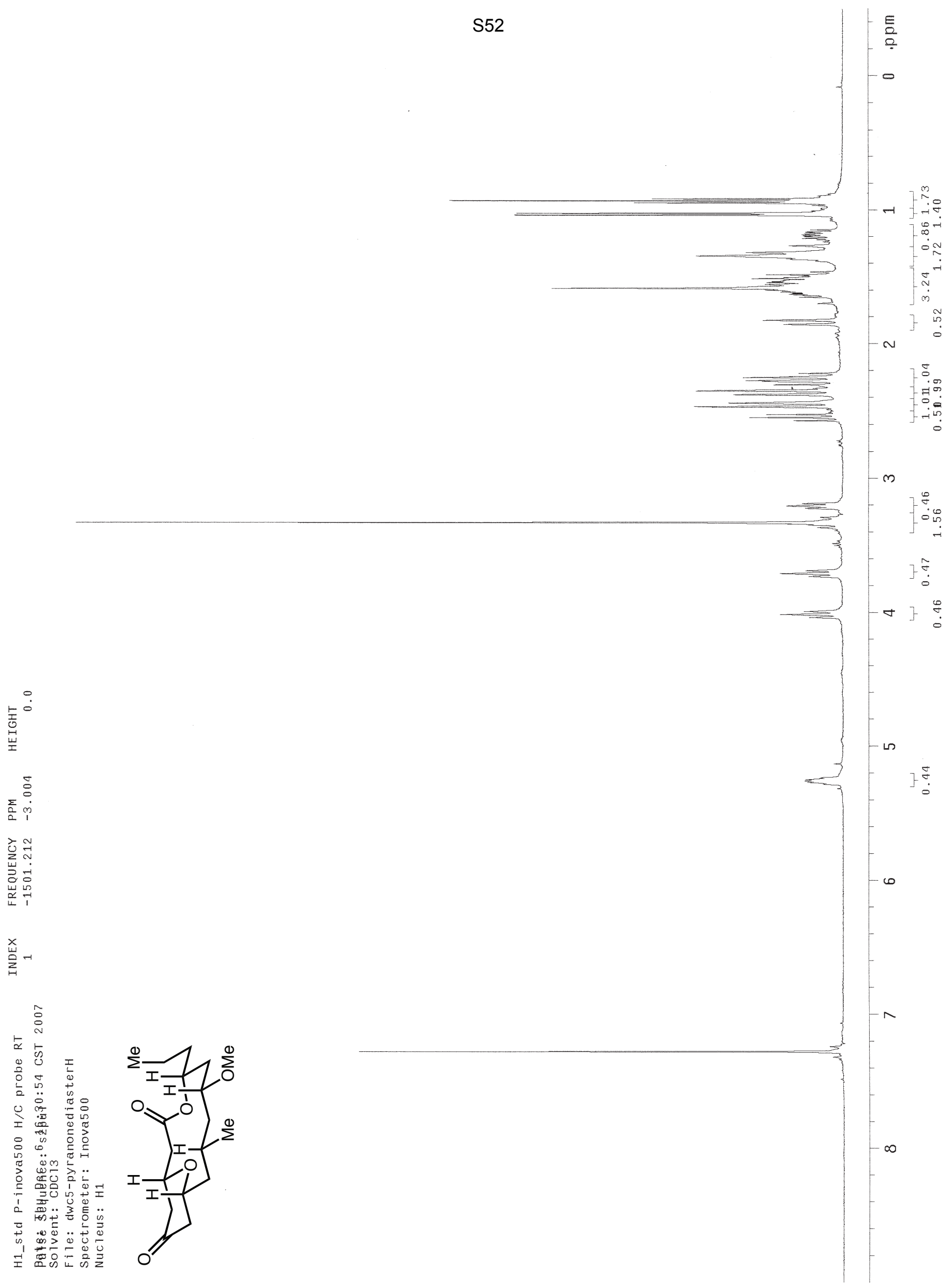




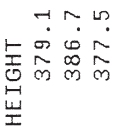

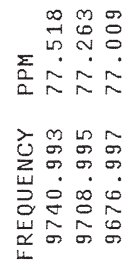

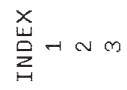

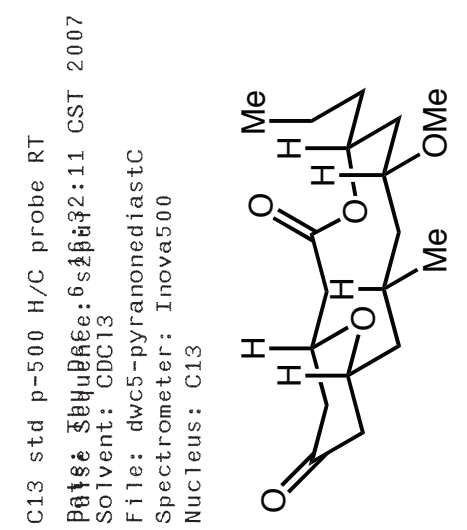




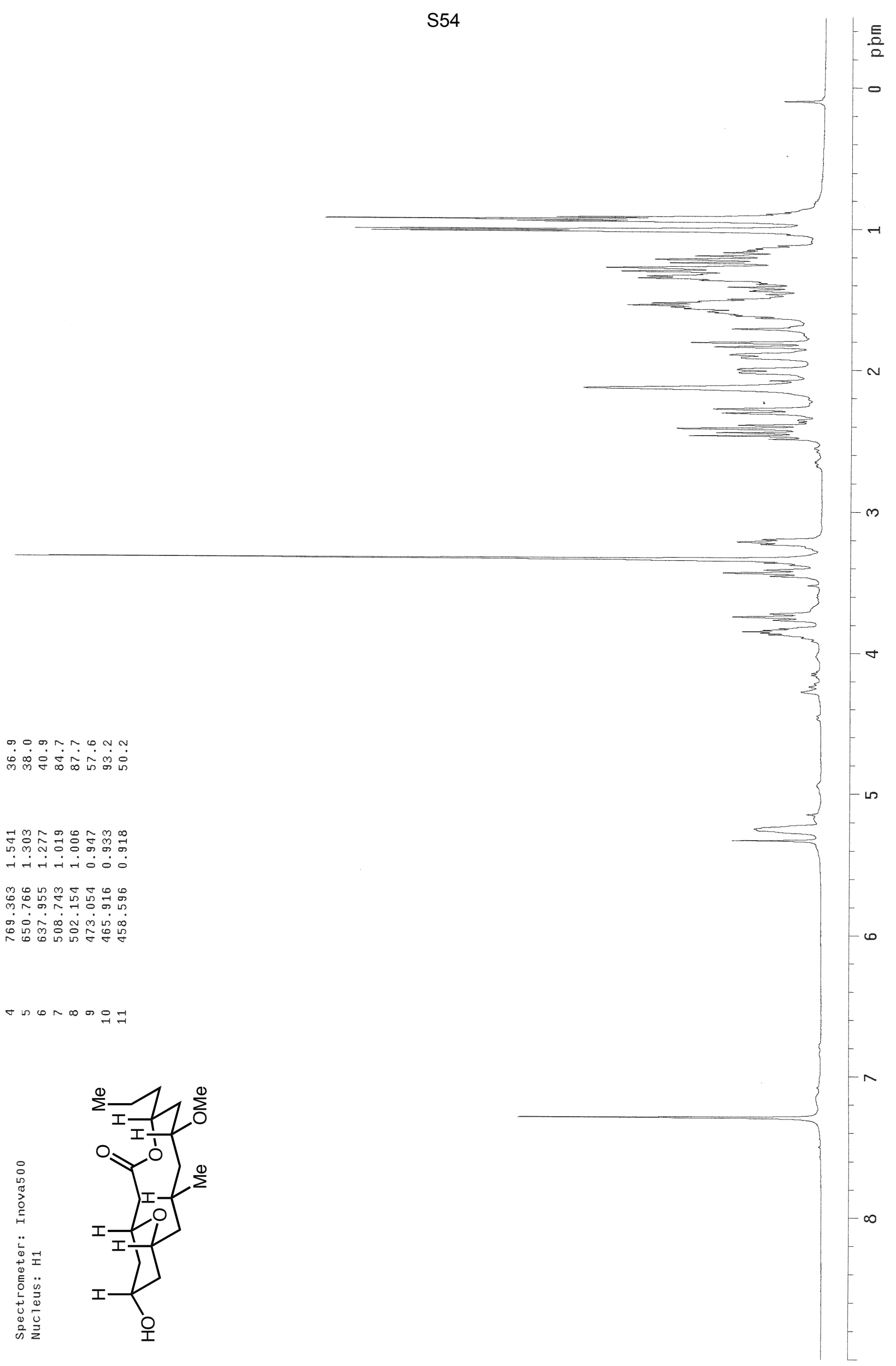




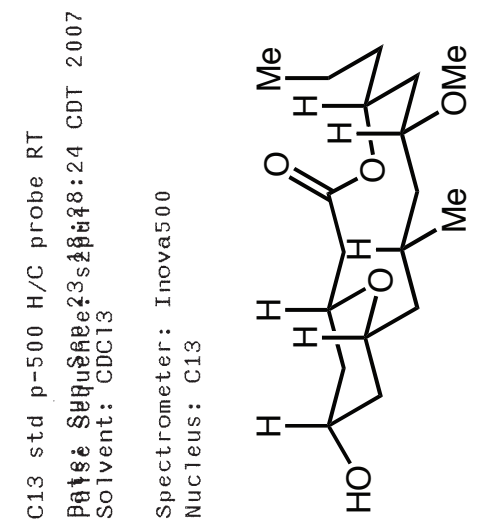




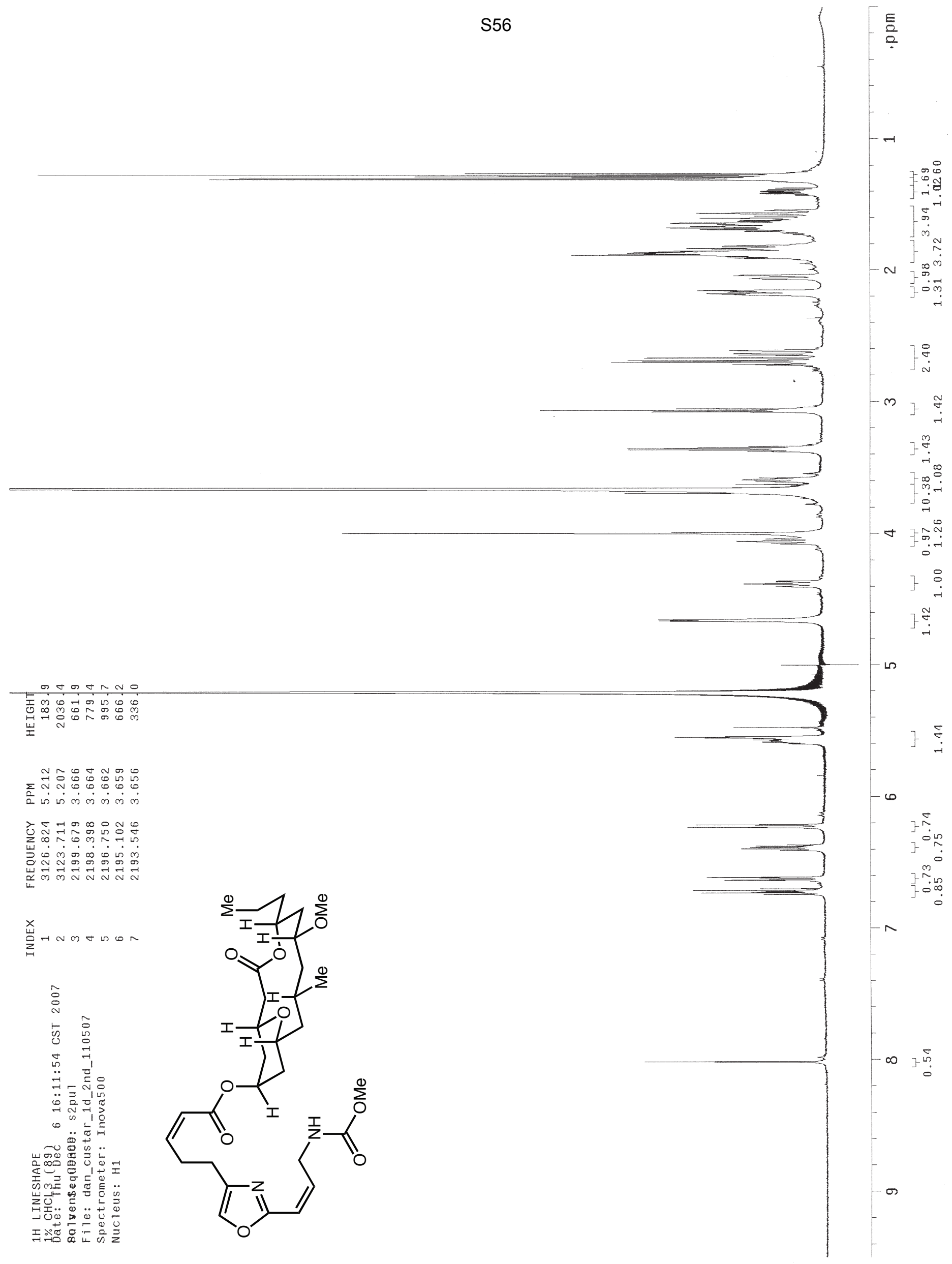




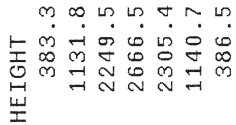

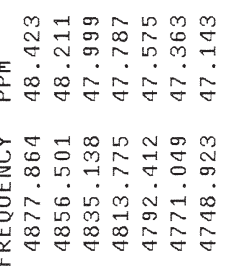

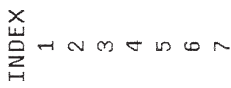
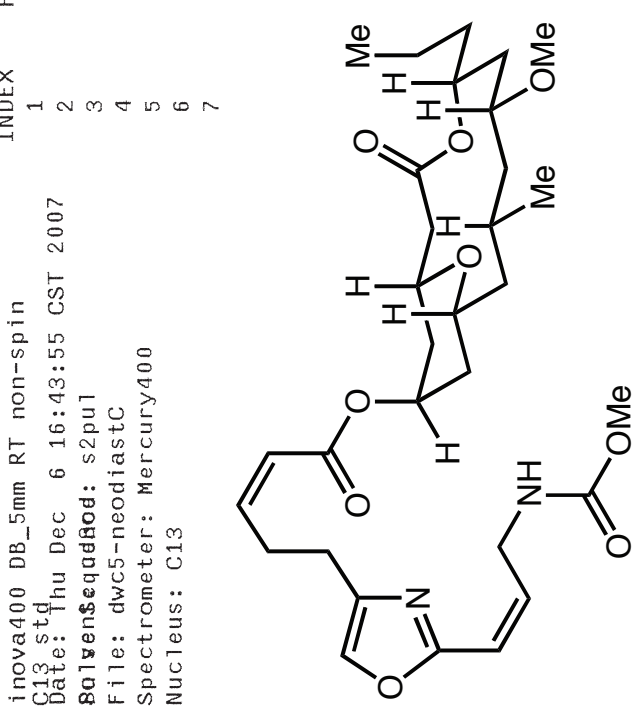


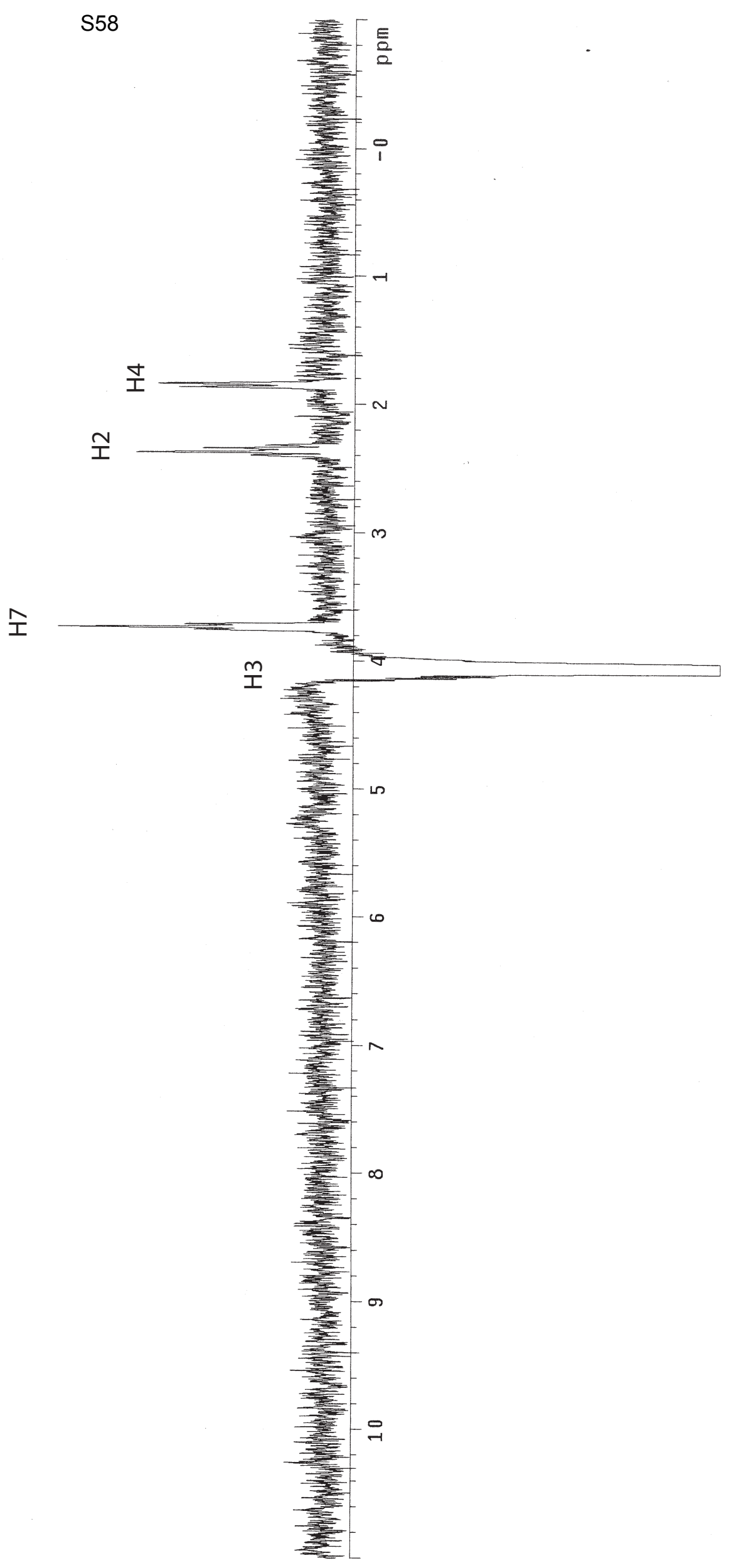




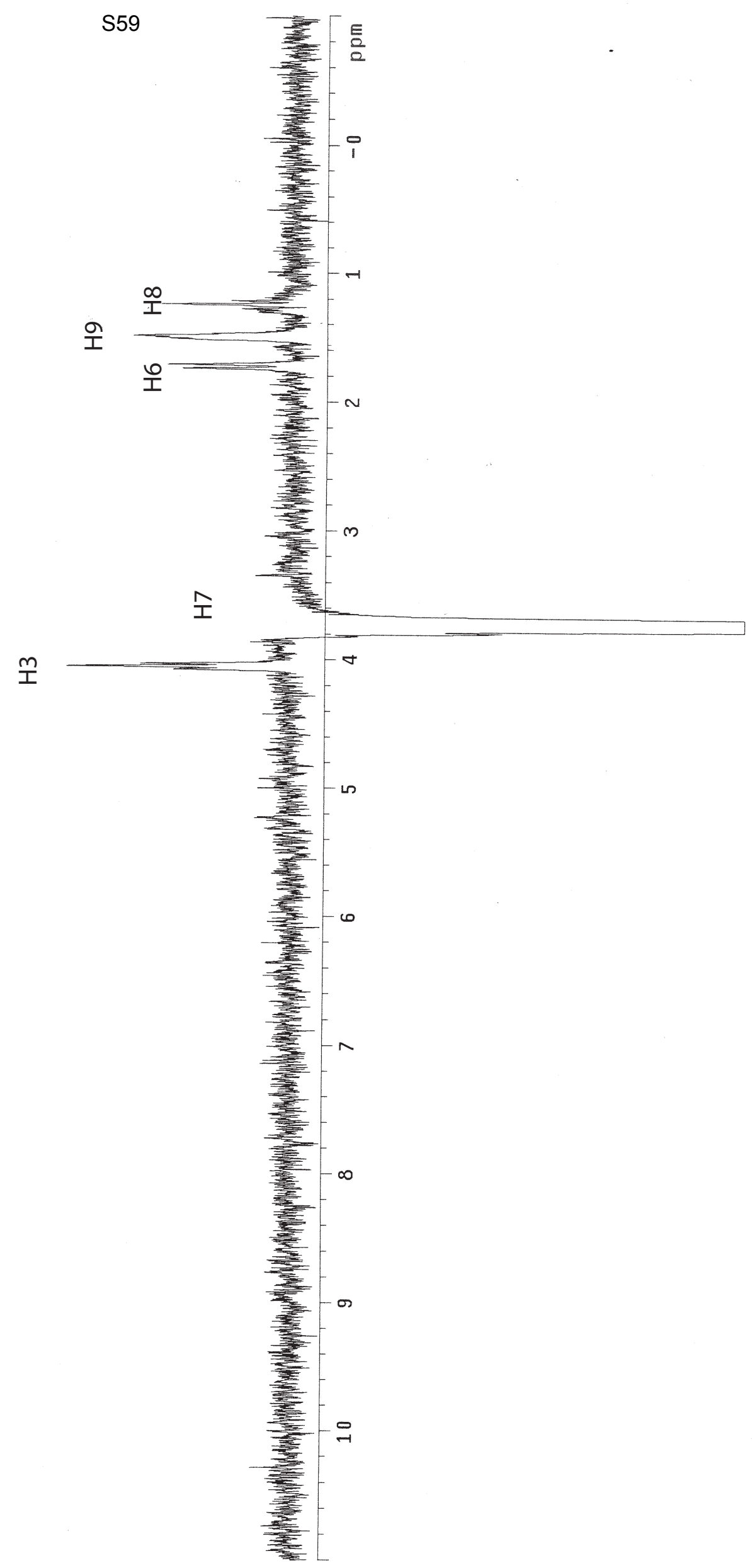

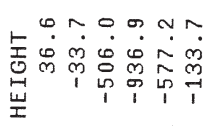

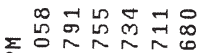

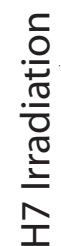

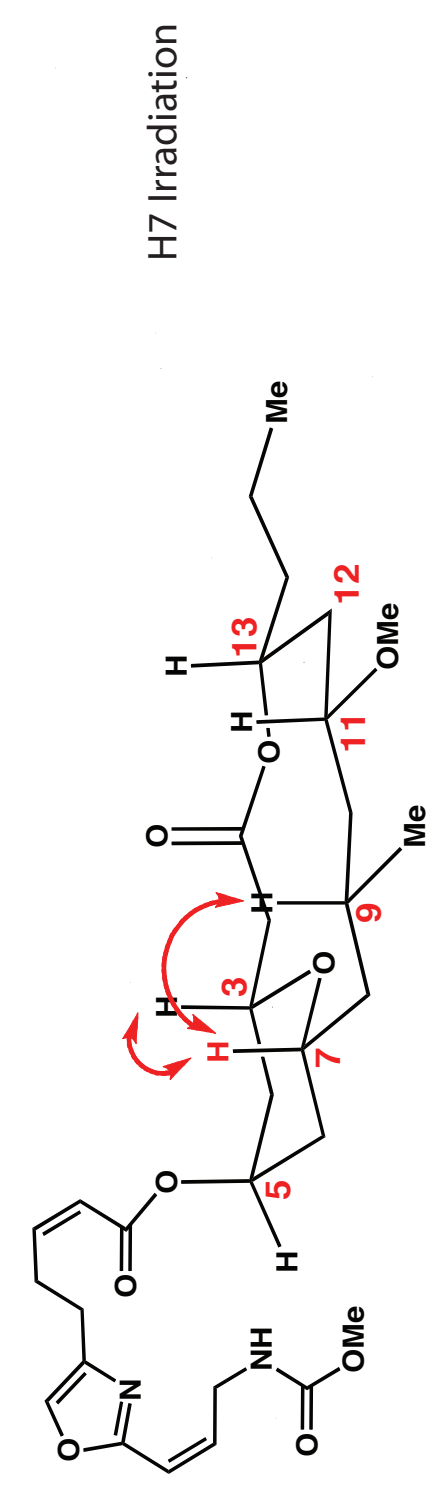

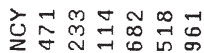

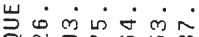

tz:

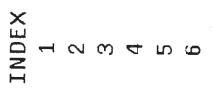

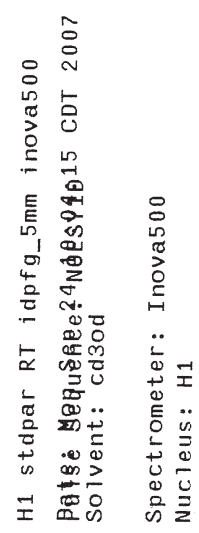




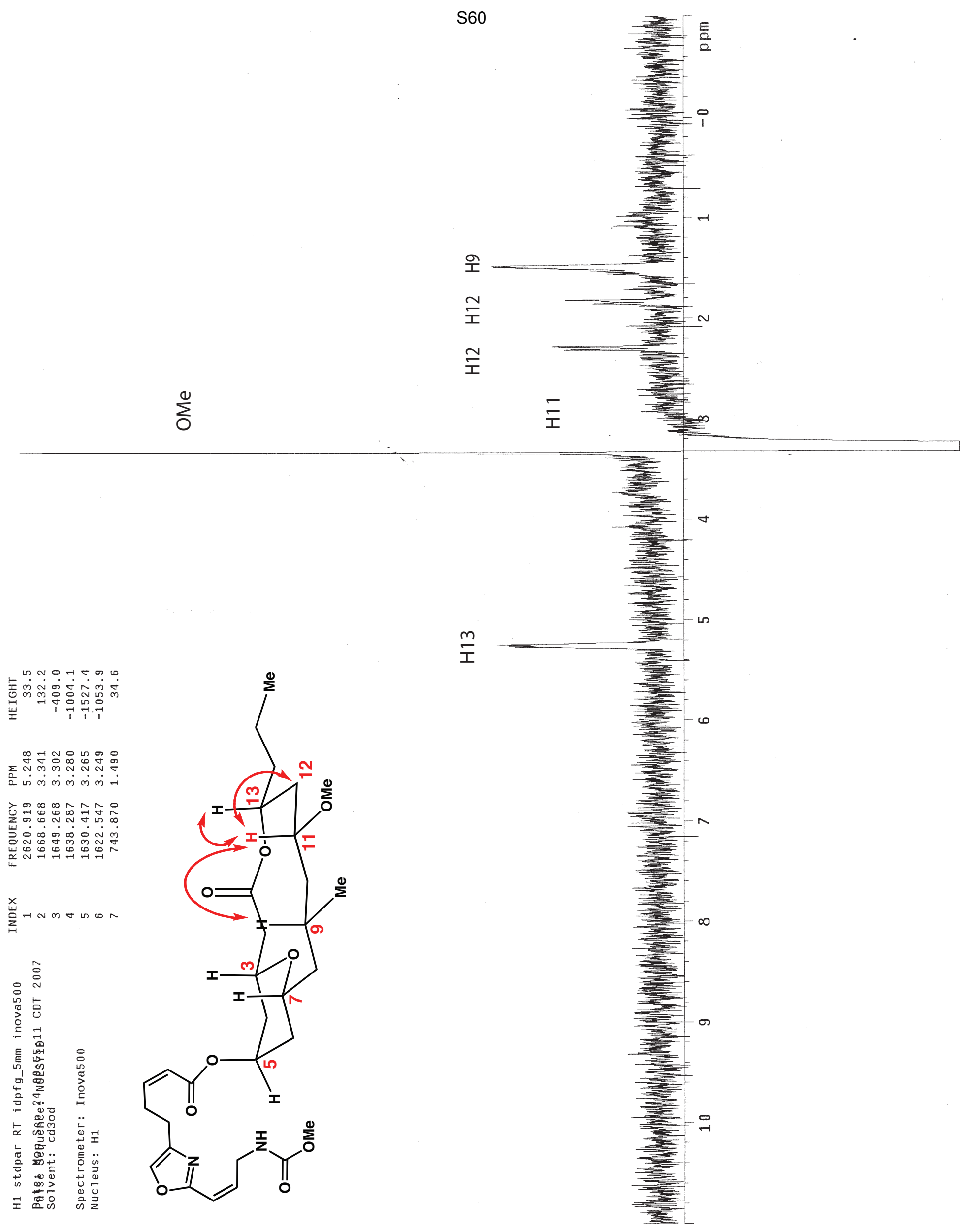




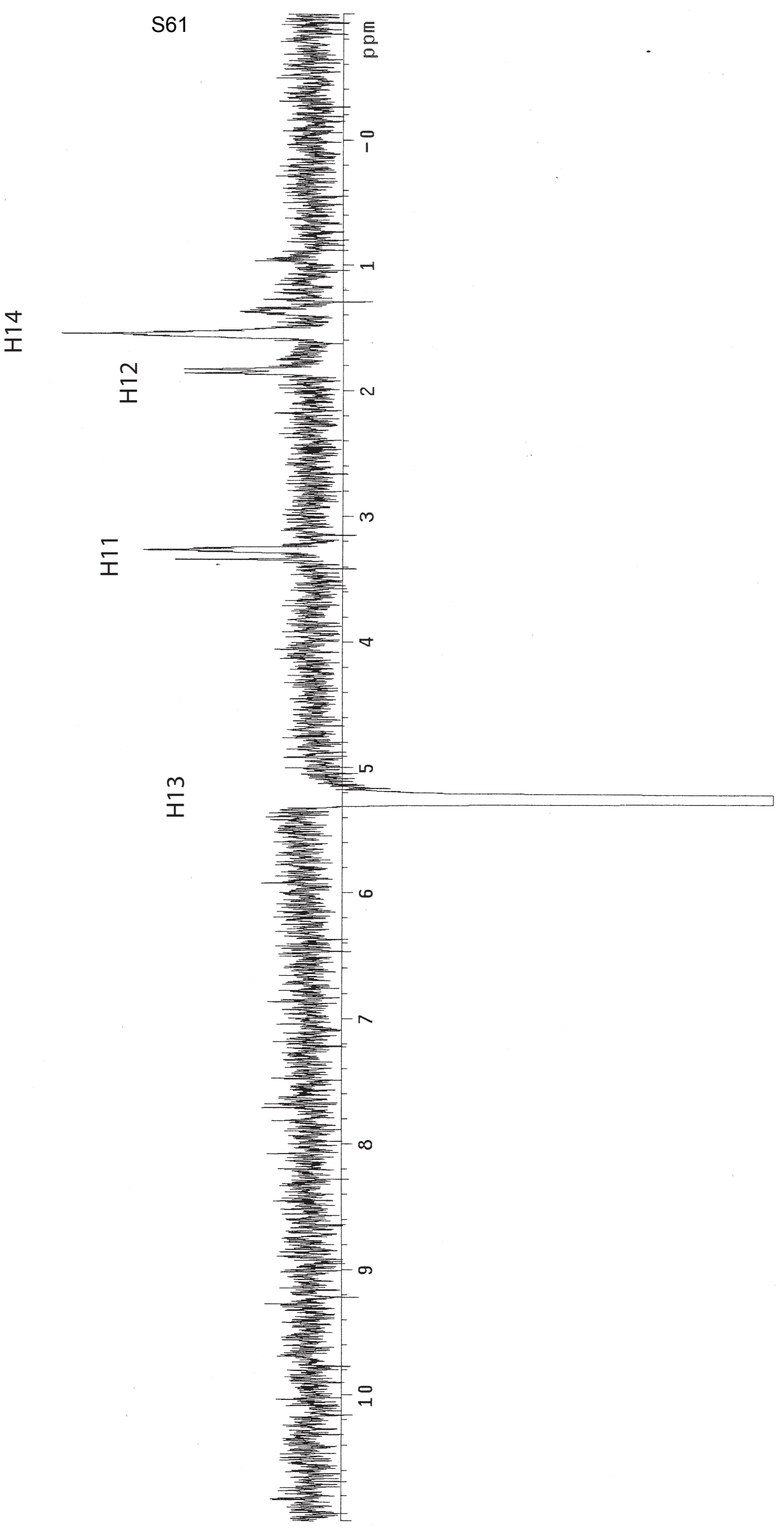

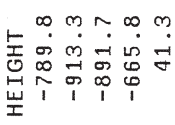

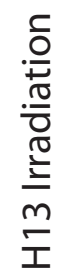

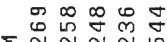

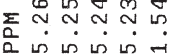

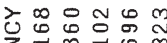

计

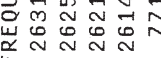

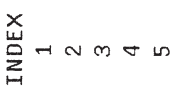
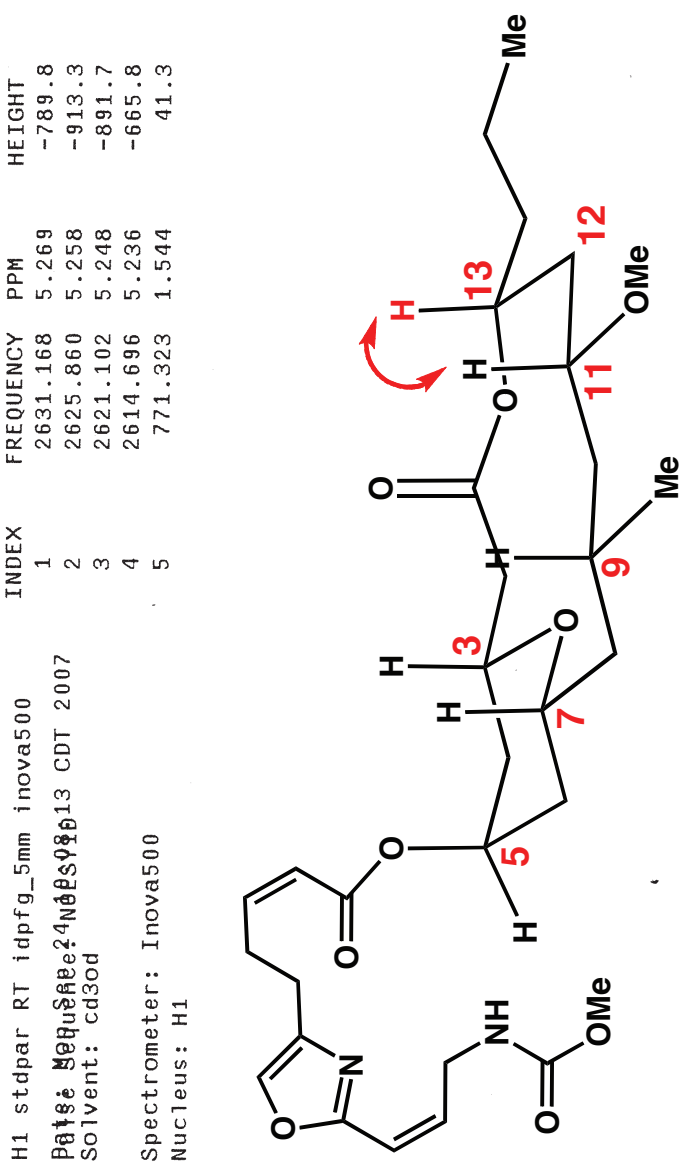


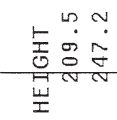
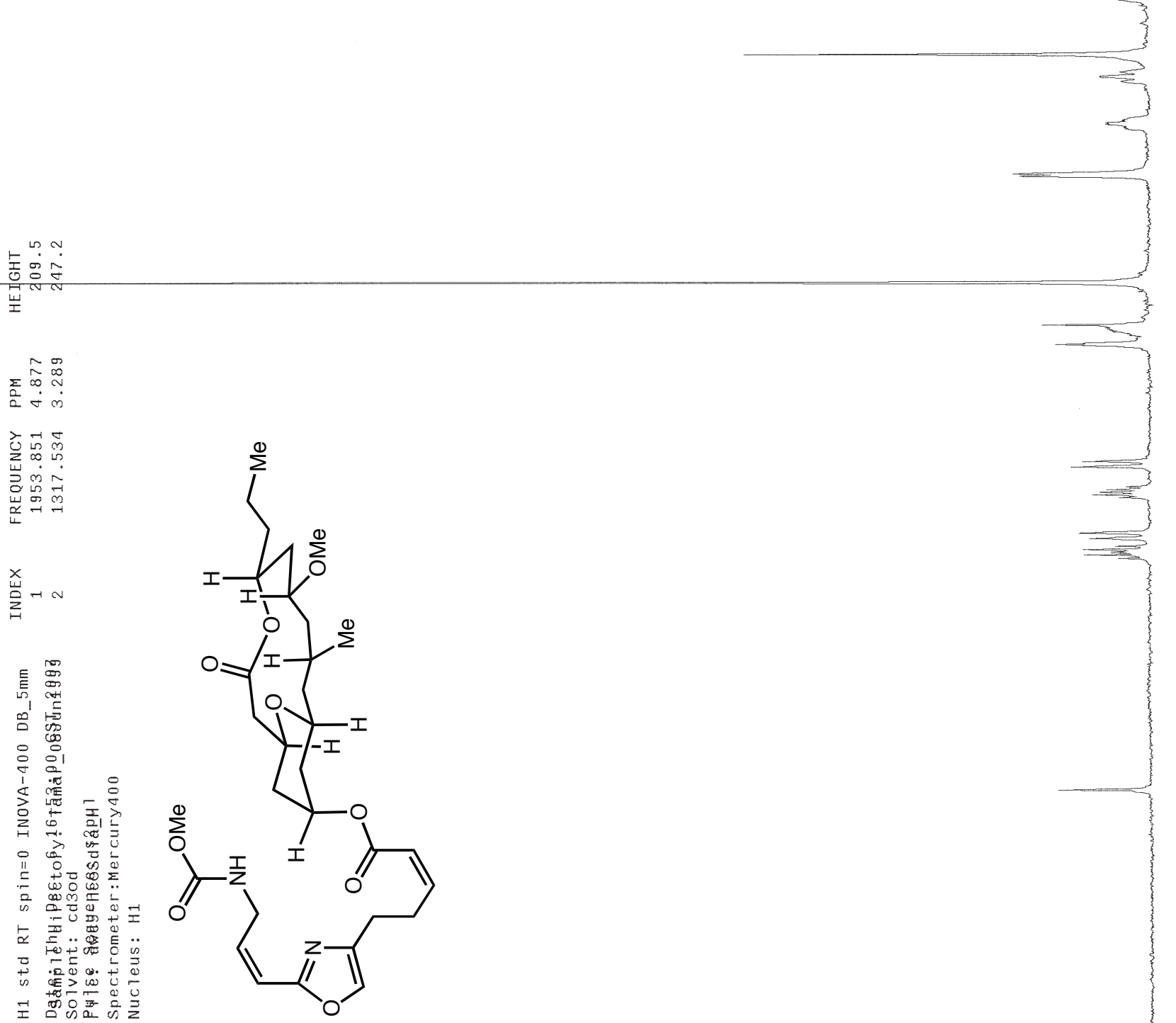


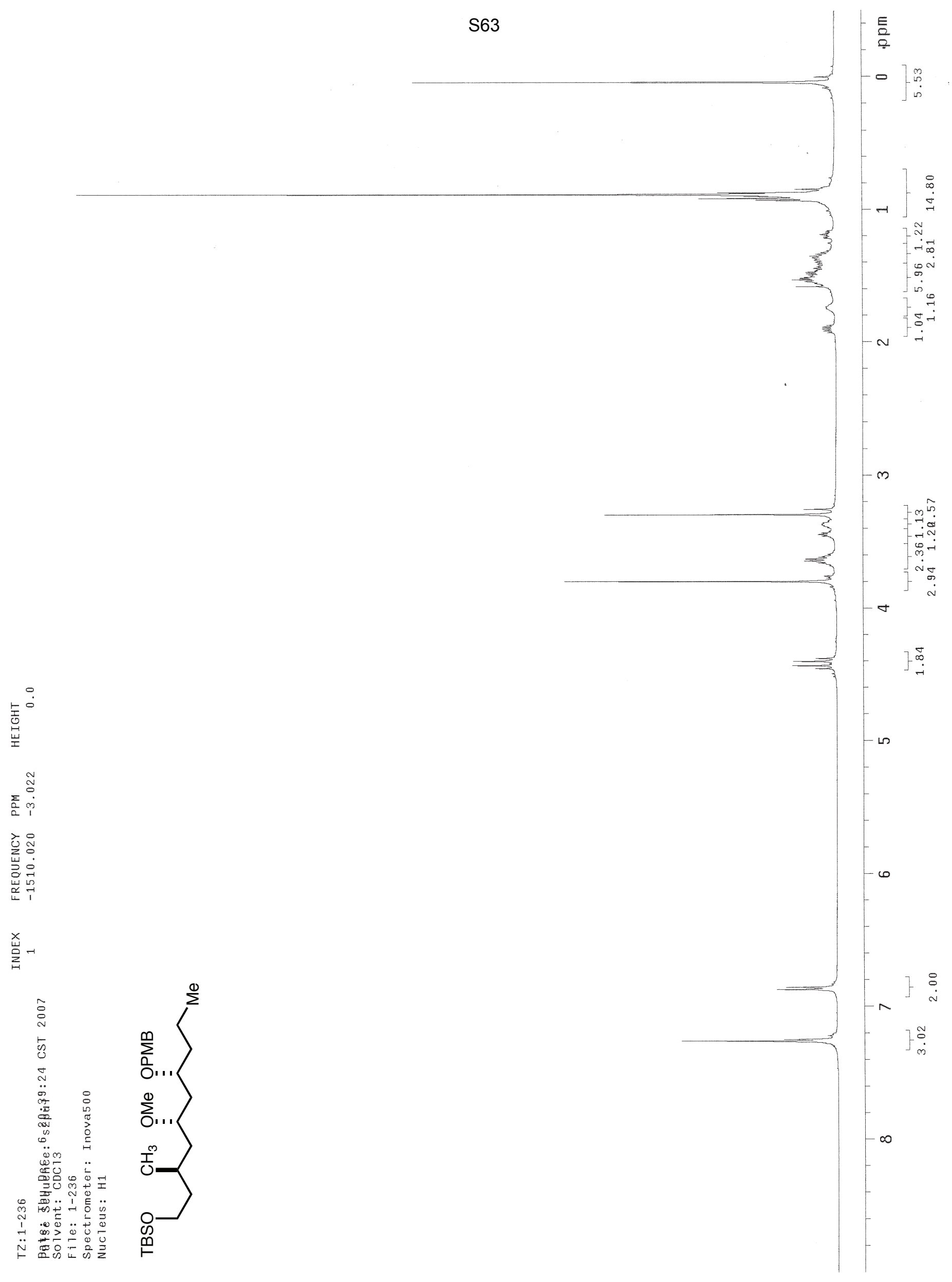




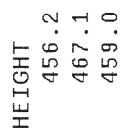

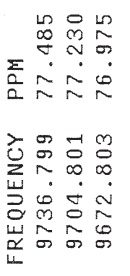

$\sum_{\substack{\mathrm{O} \\ \mathrm{u}}}^{x} \sim N$

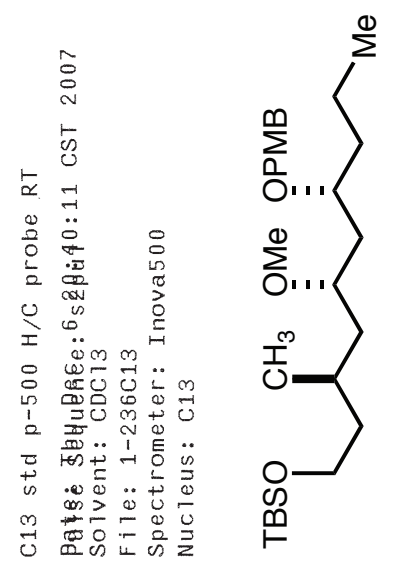




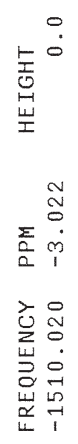

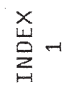

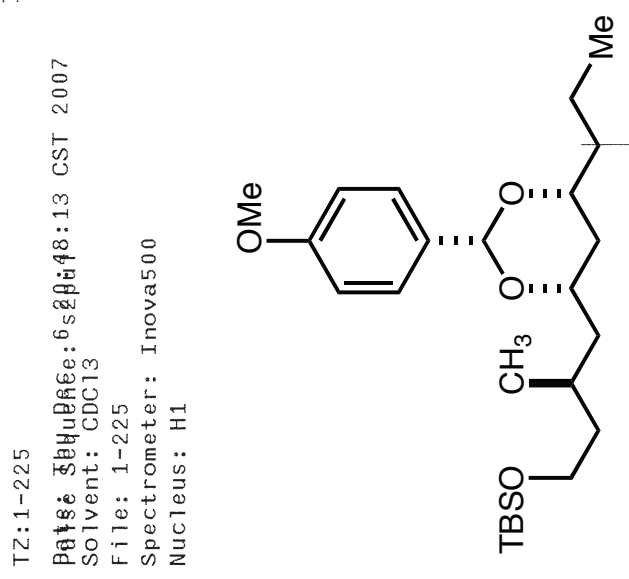

$m$

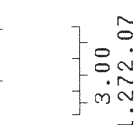




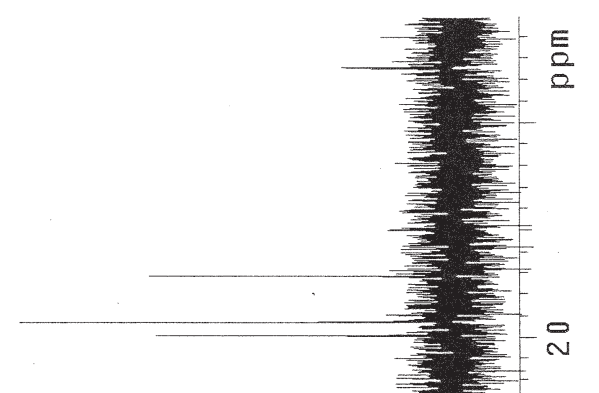

器影影

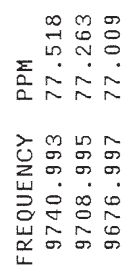

$\sum_{i=1}^{x}-N m$

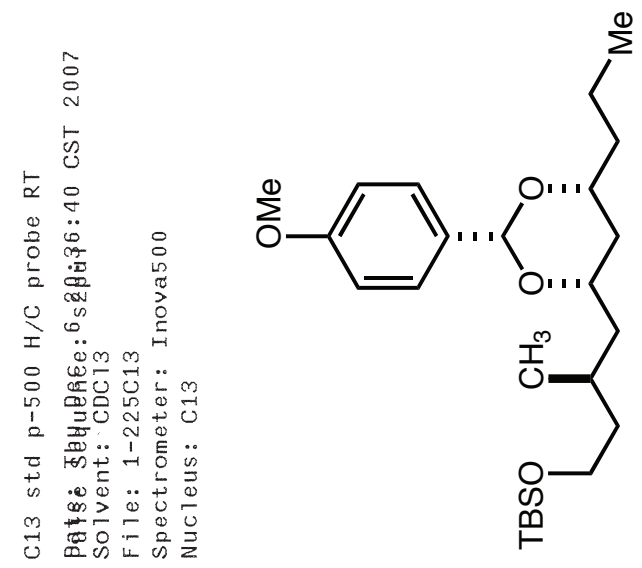




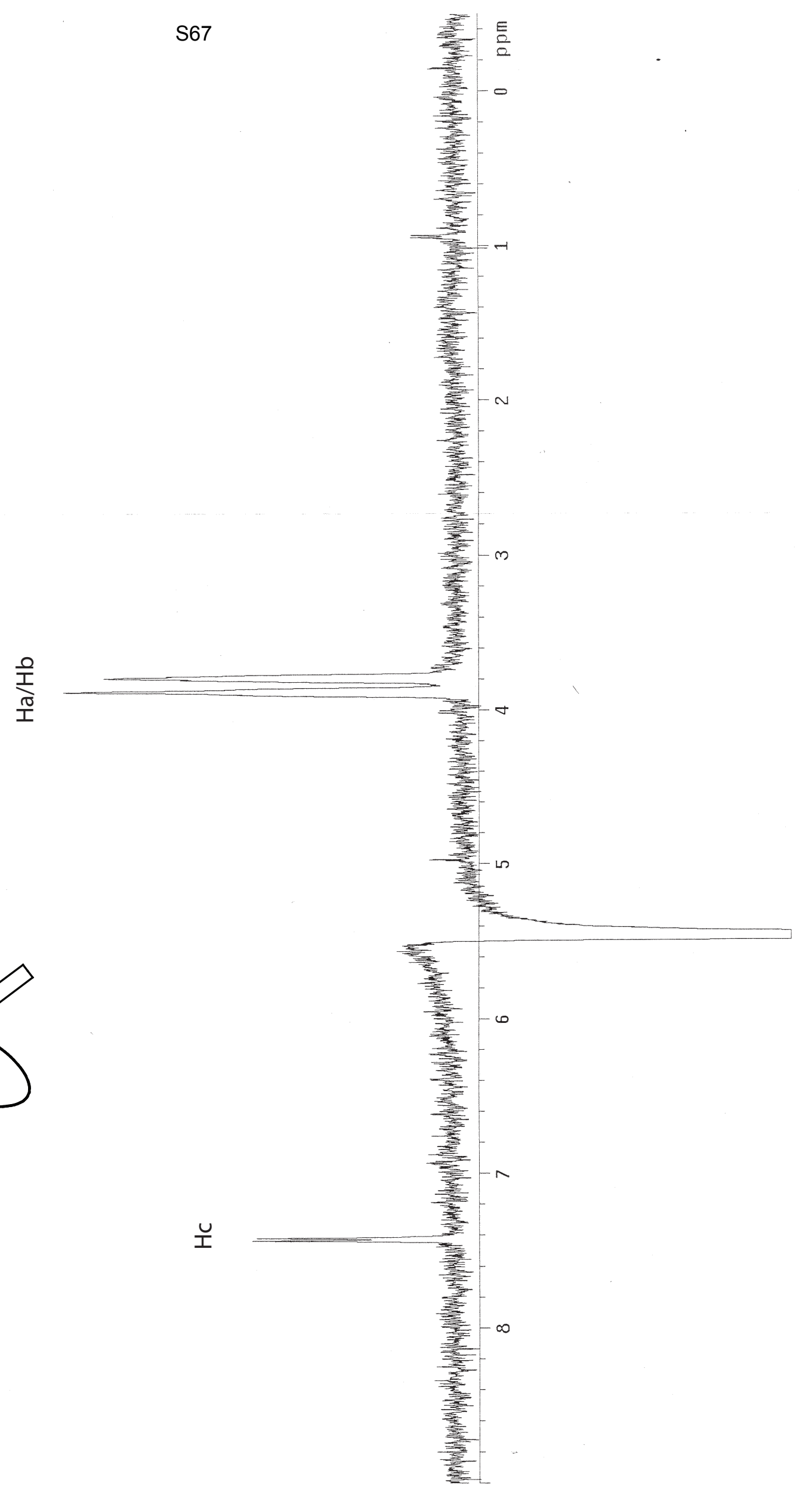




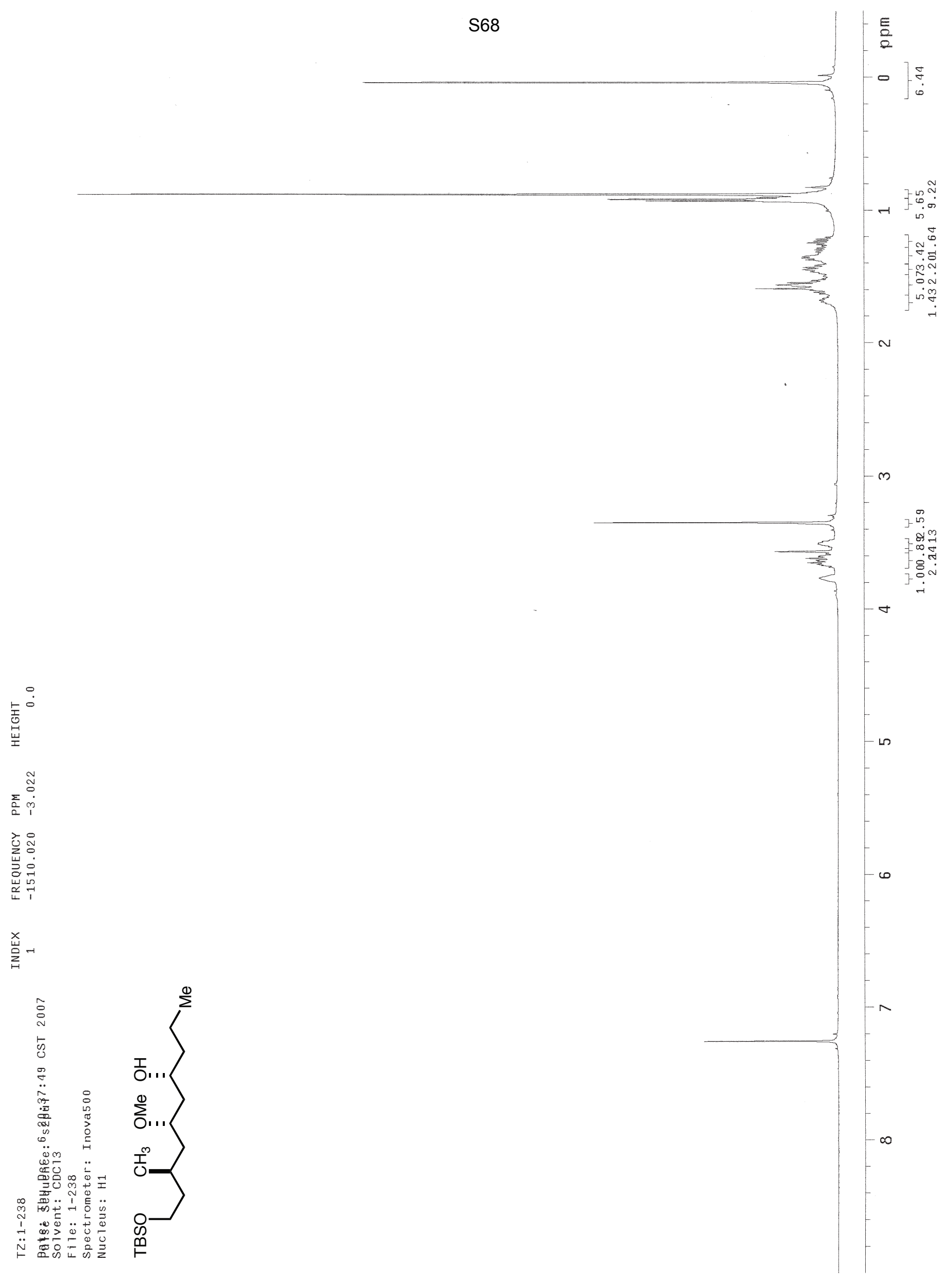




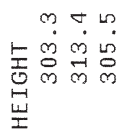

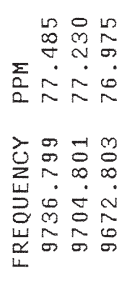

$\sum_{\text {嵌 }}^{x} N m$

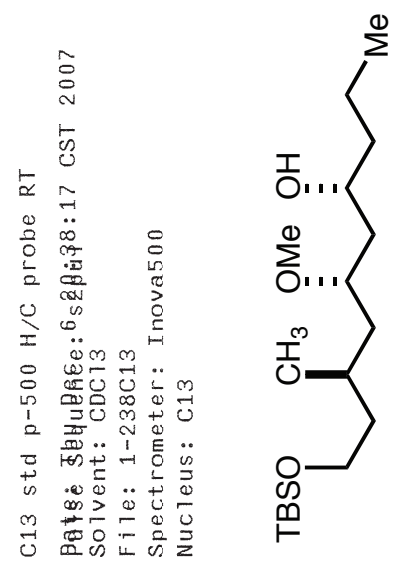




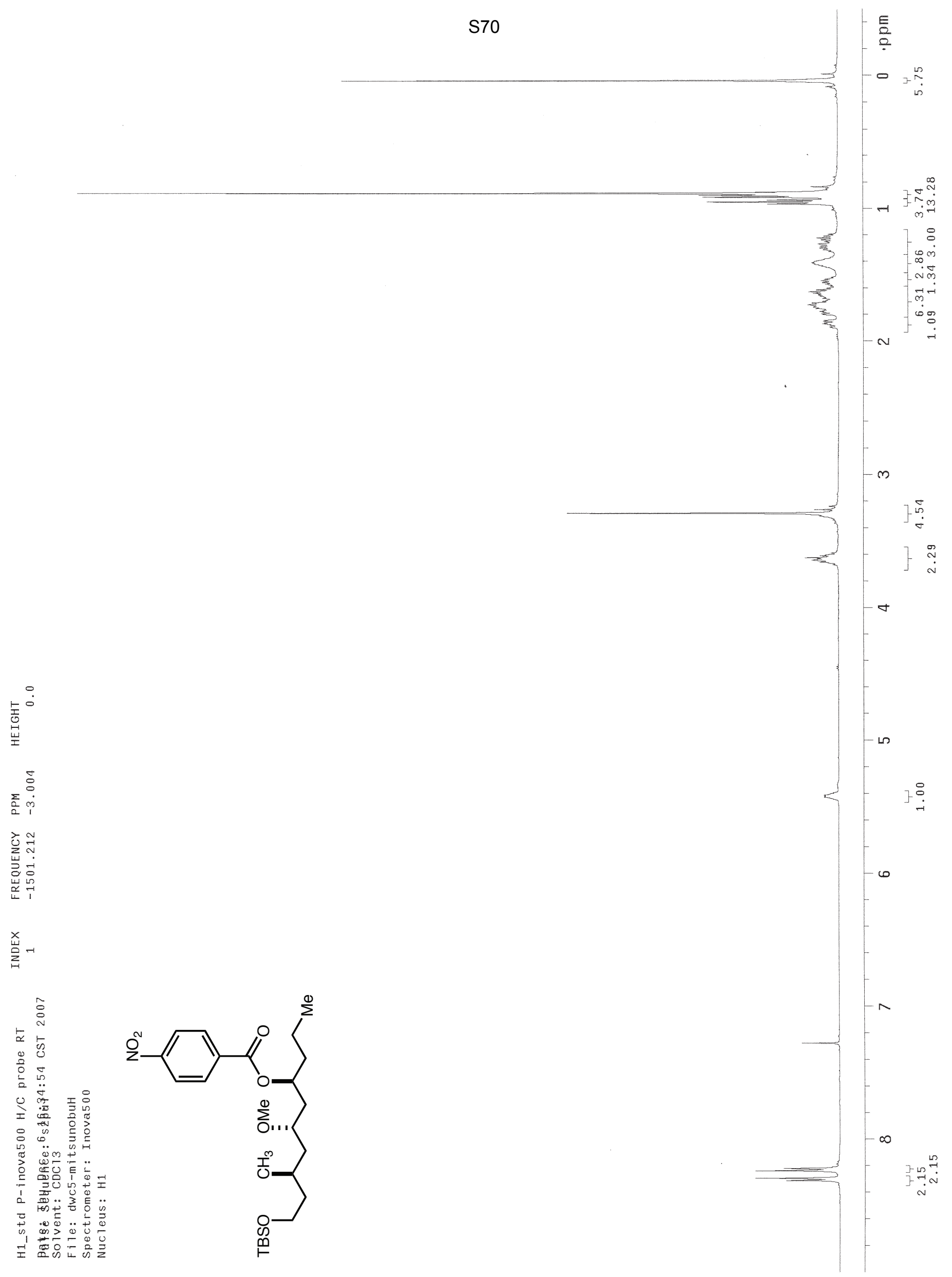




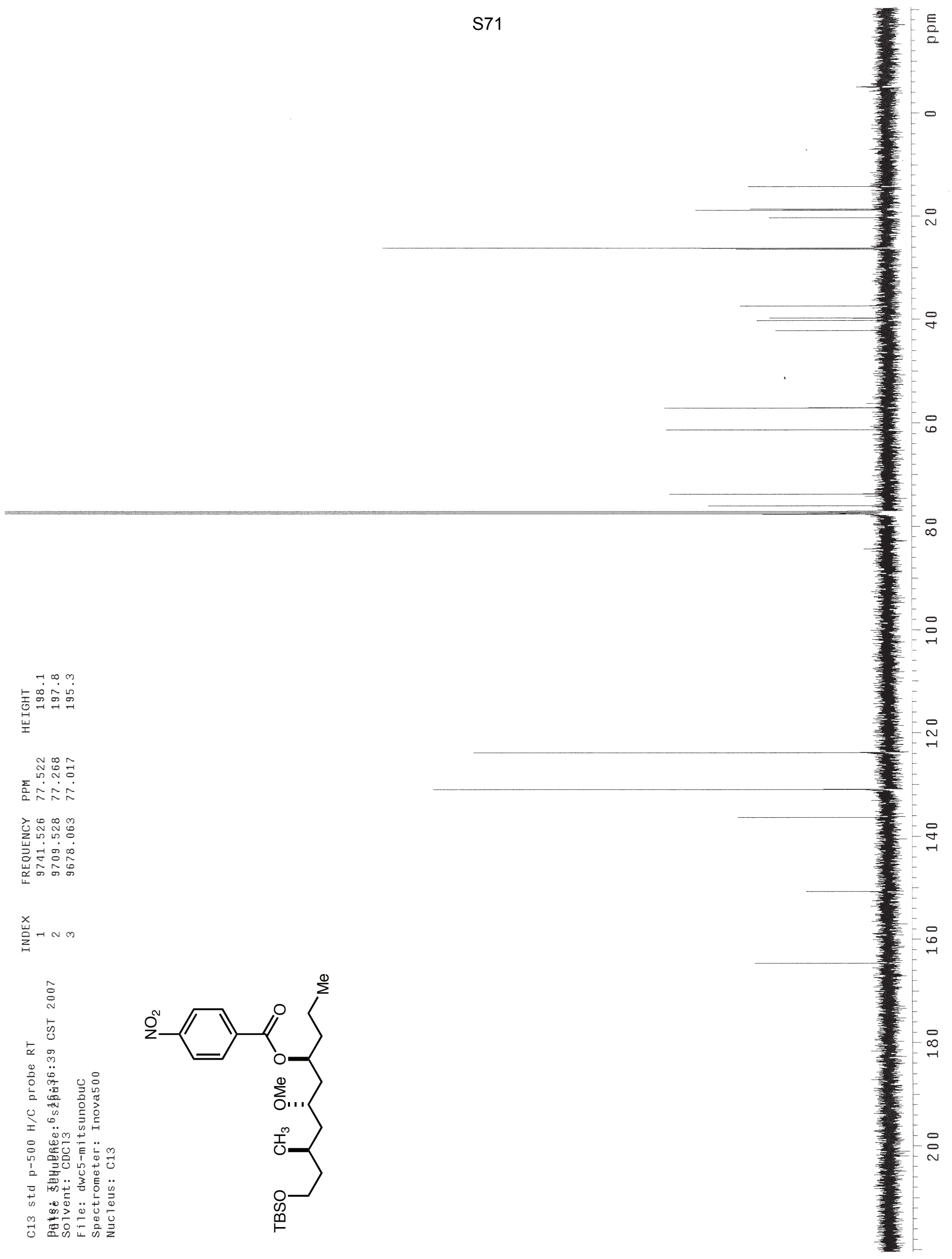




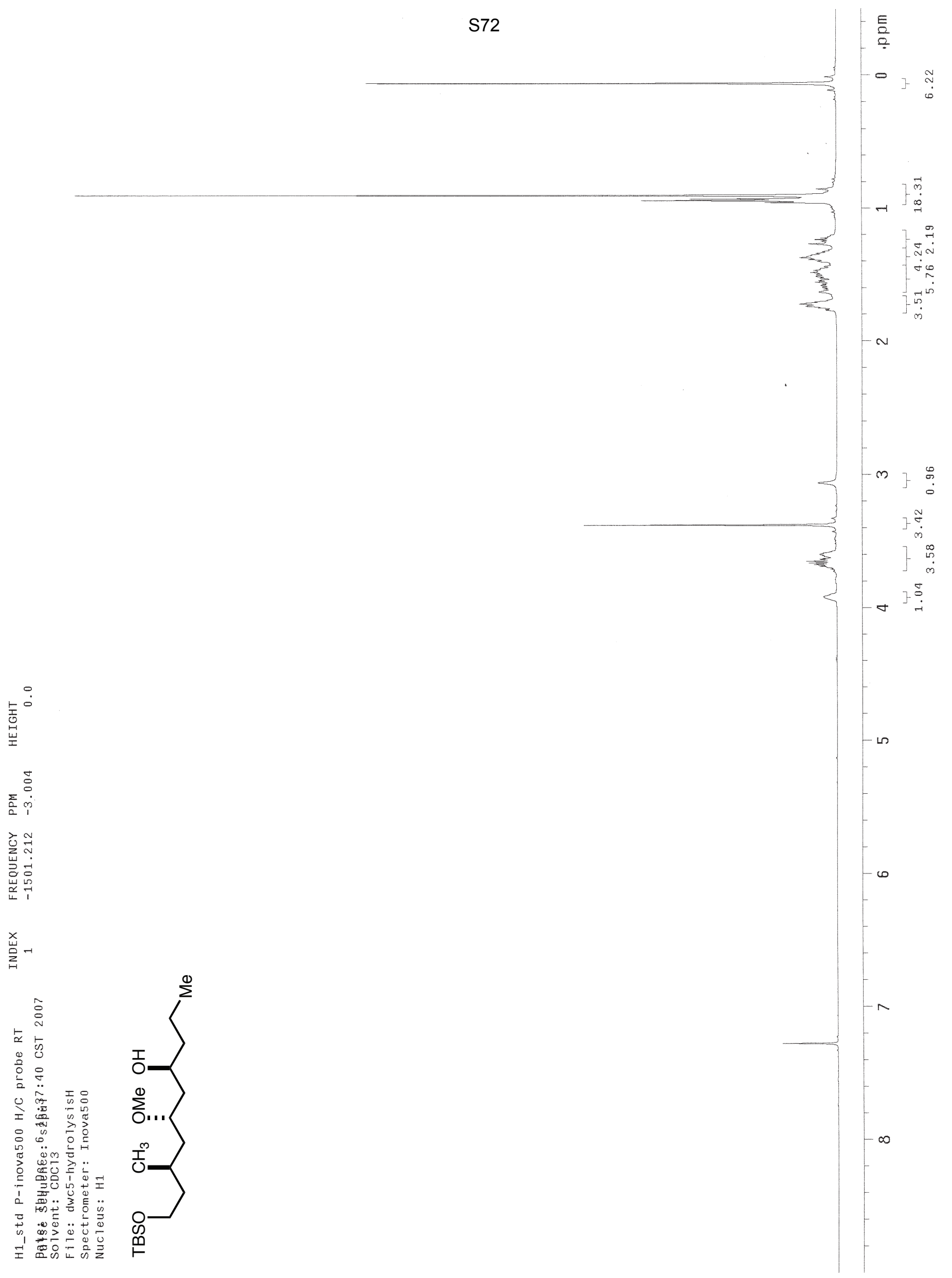




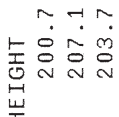

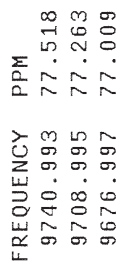

$\sum_{i=1}^{x} \dashv N m$

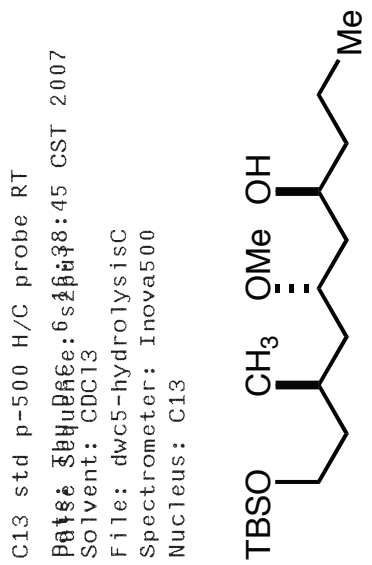




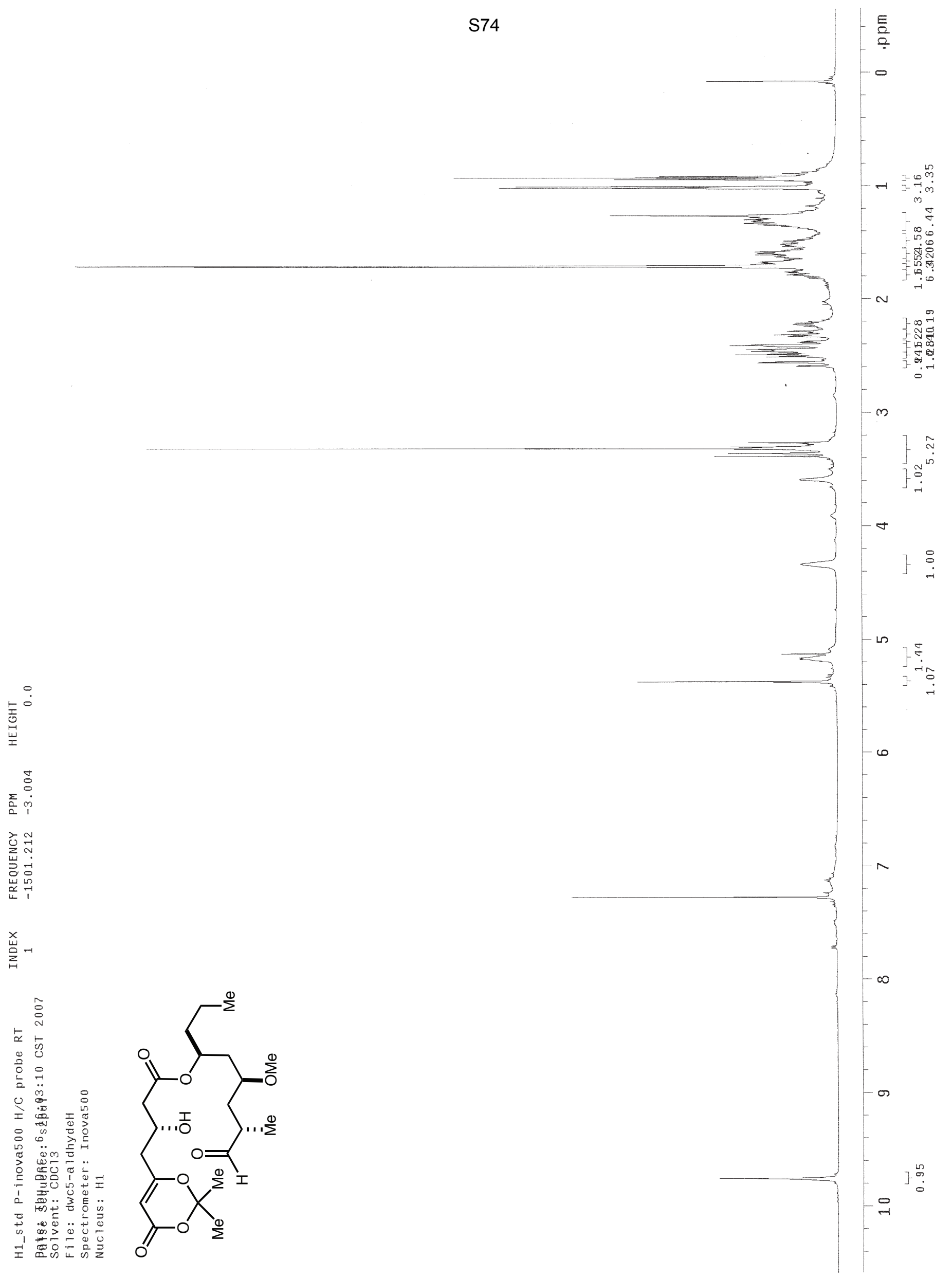




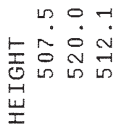

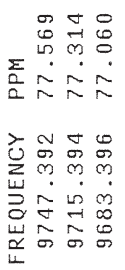

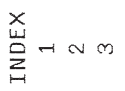
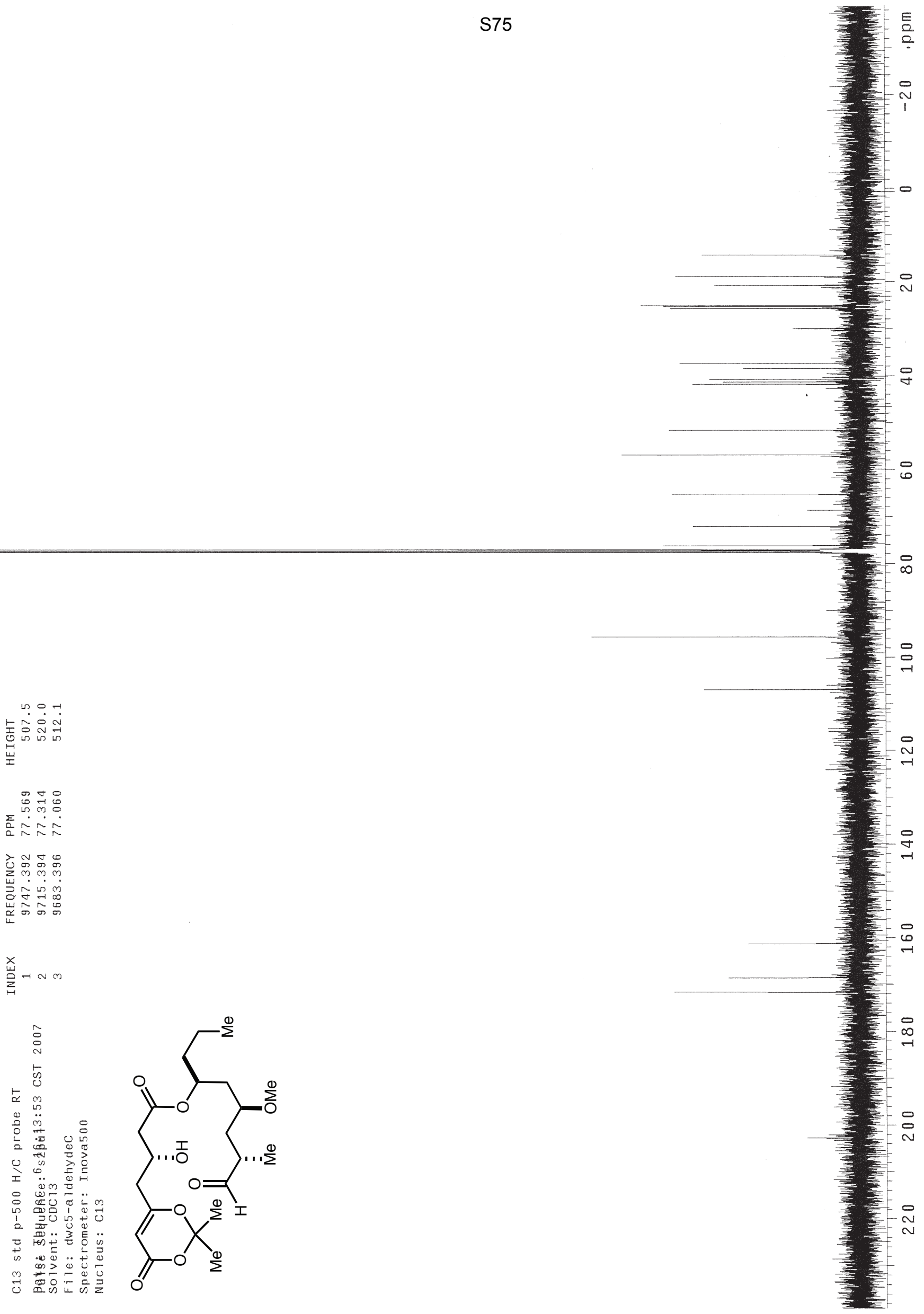


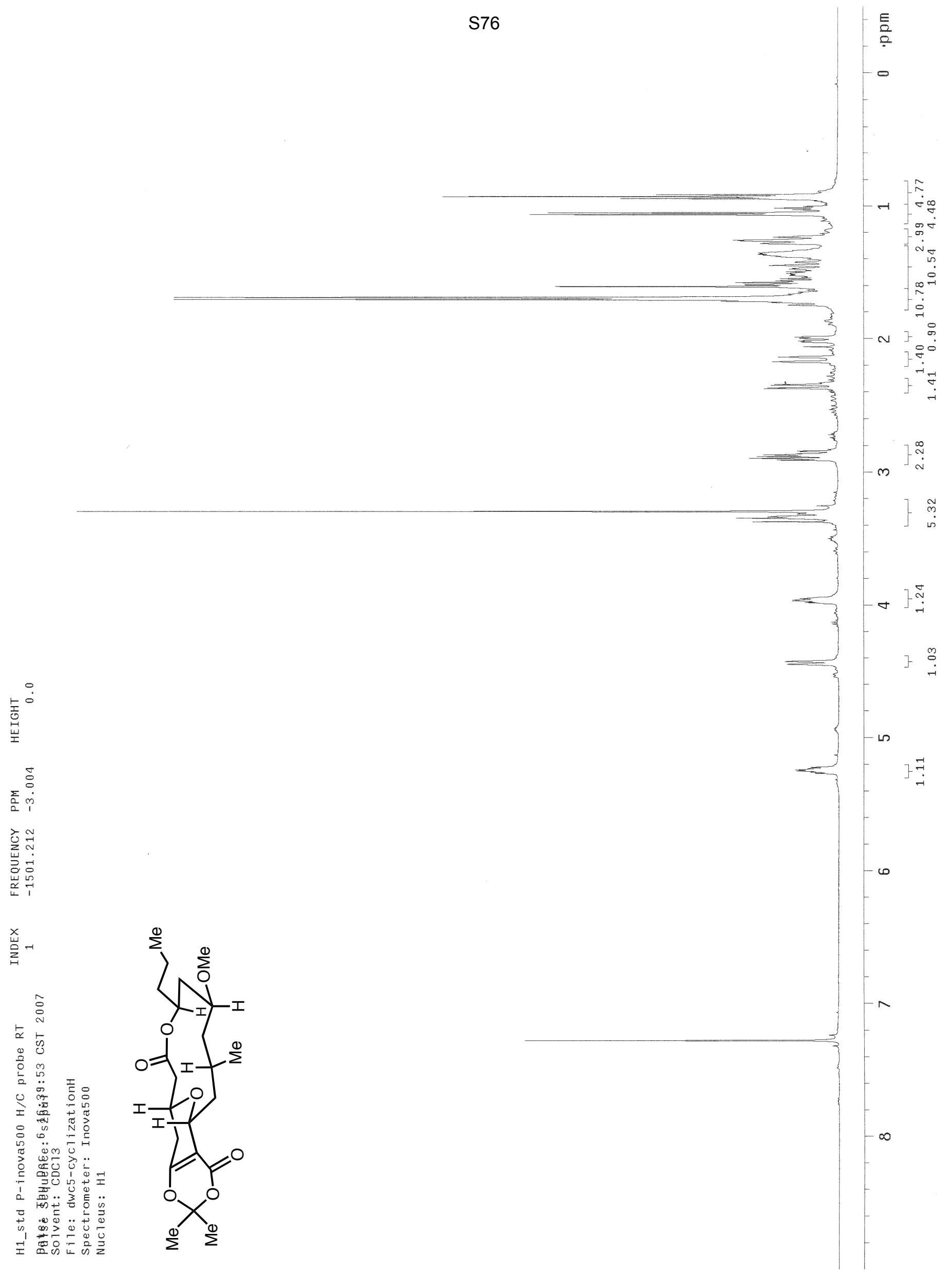




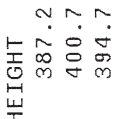

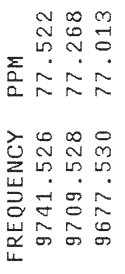
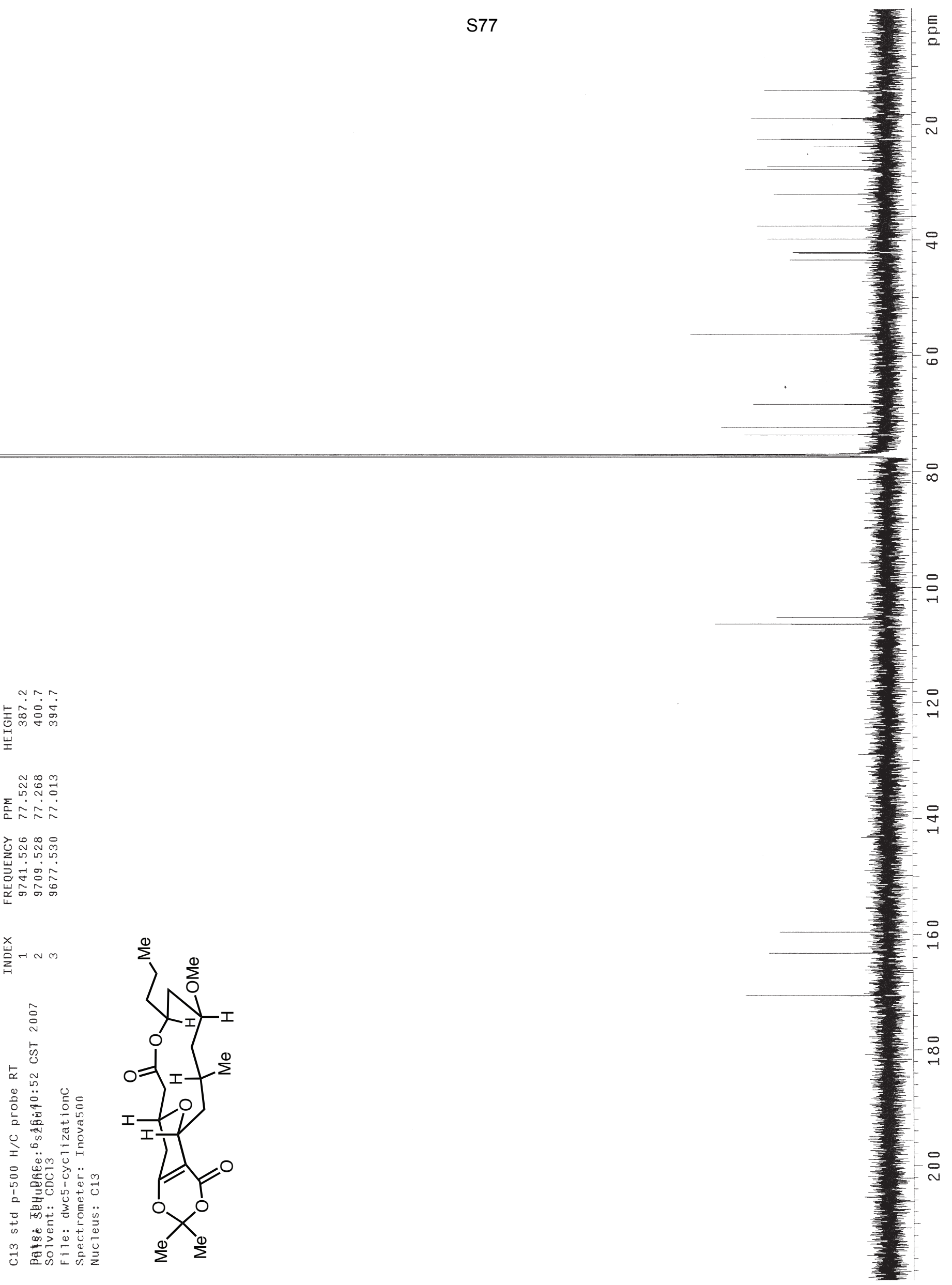


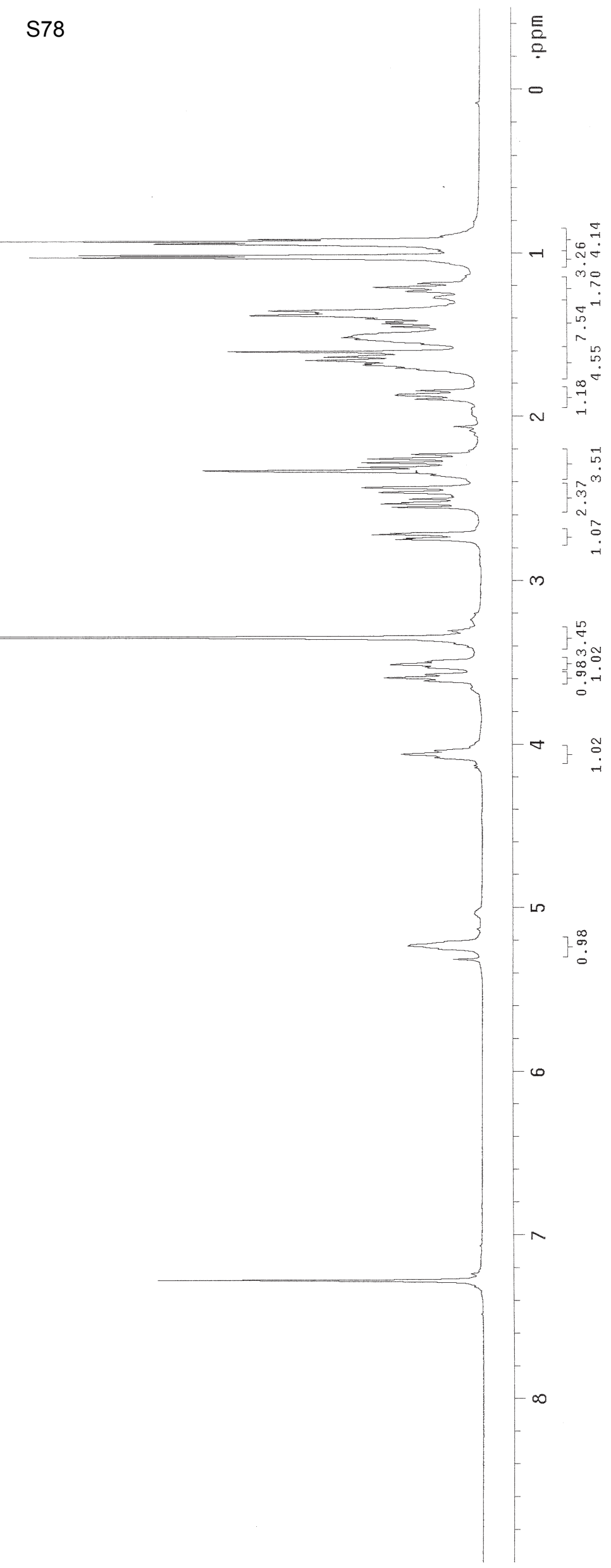




$$
1
$$




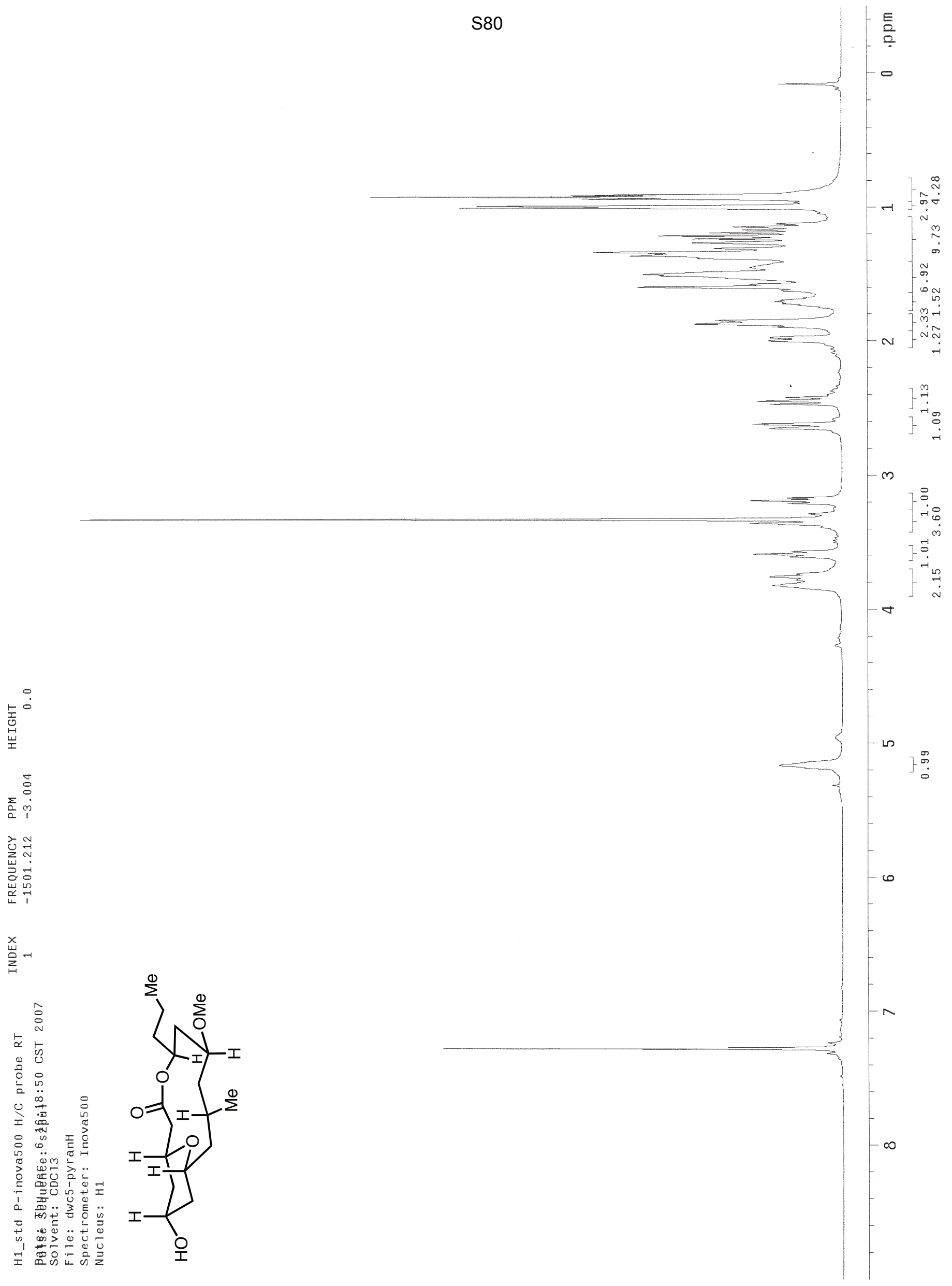




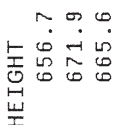

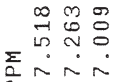

高交京

टे

岁字京定

twa

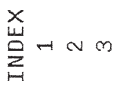
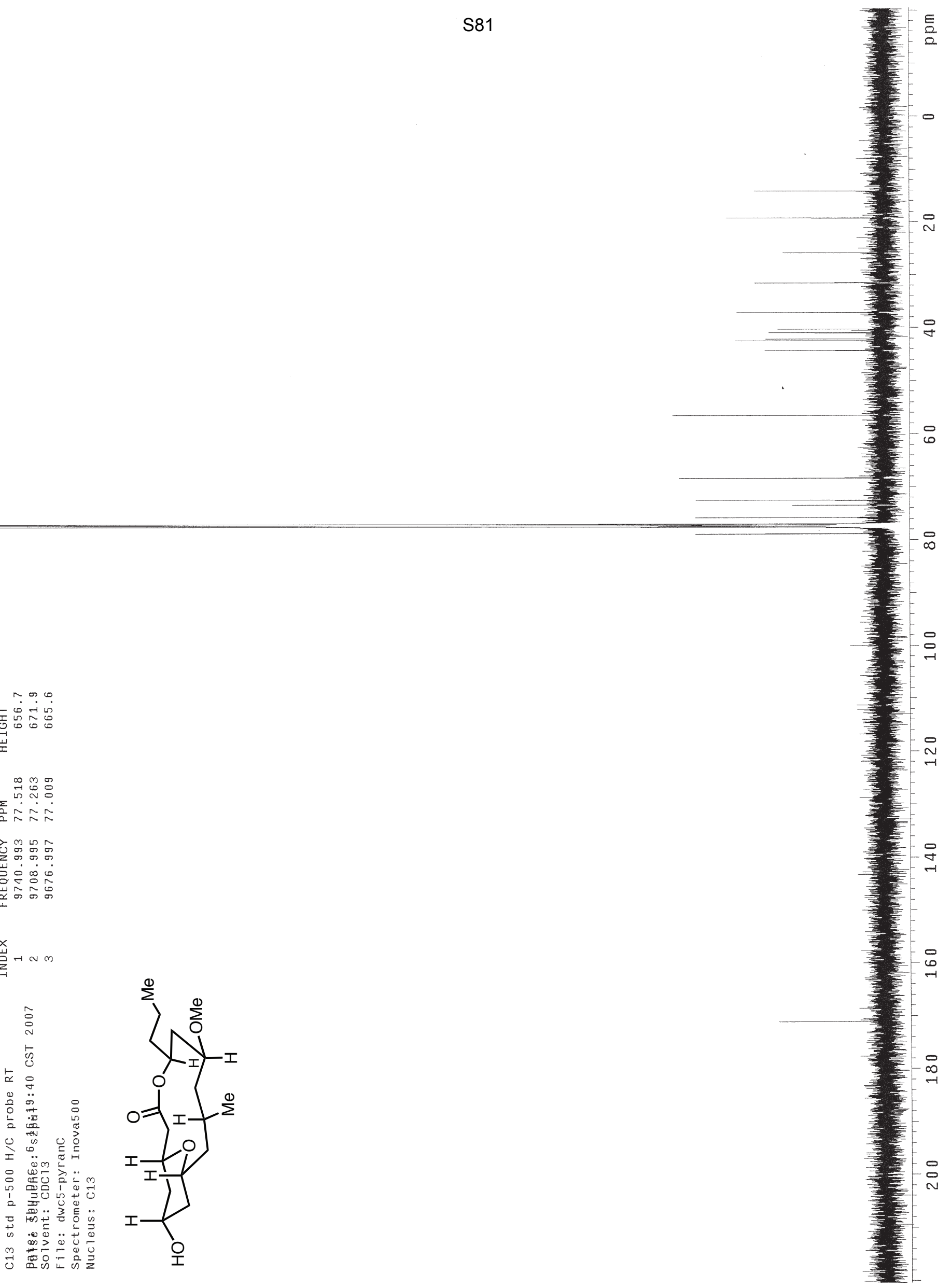


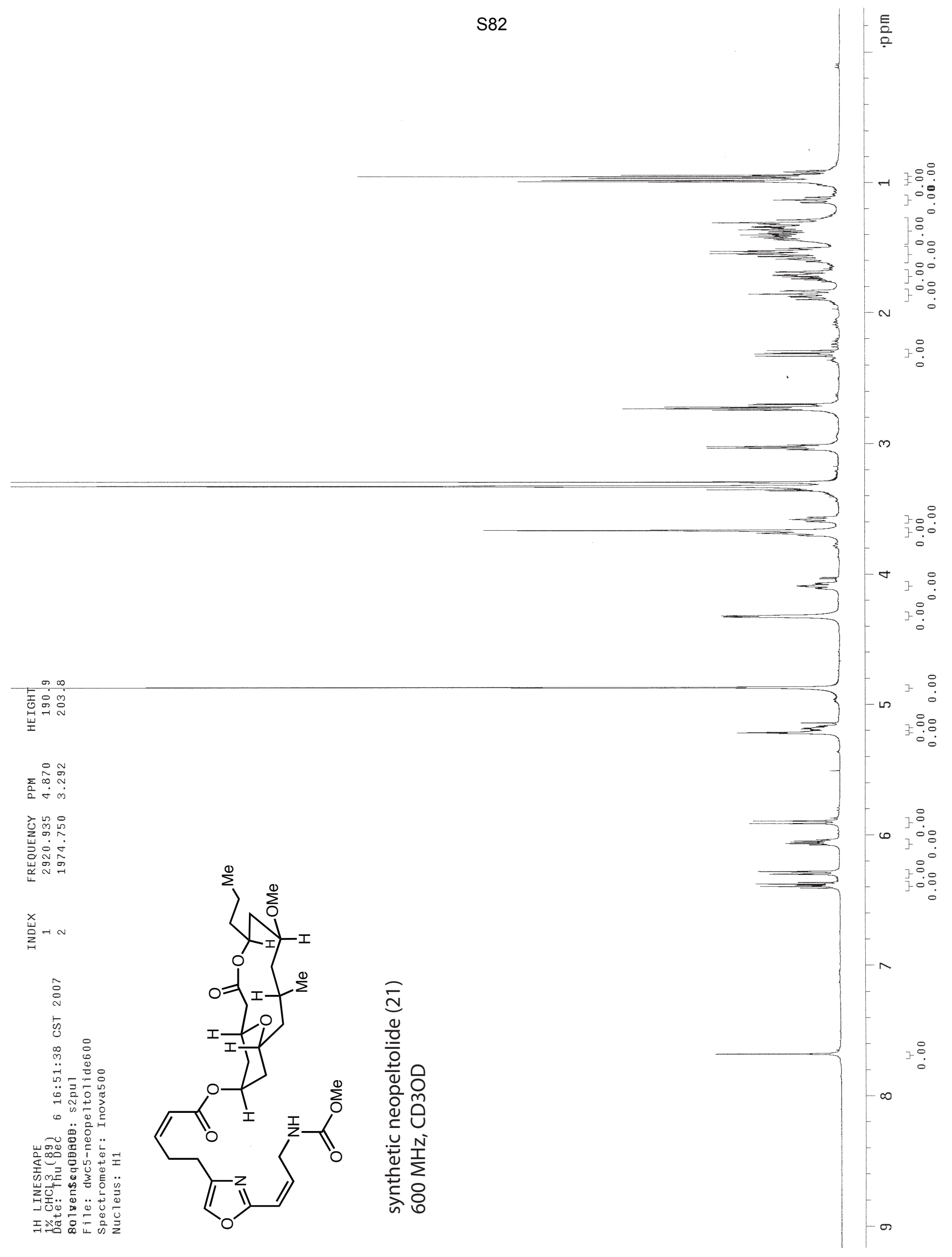




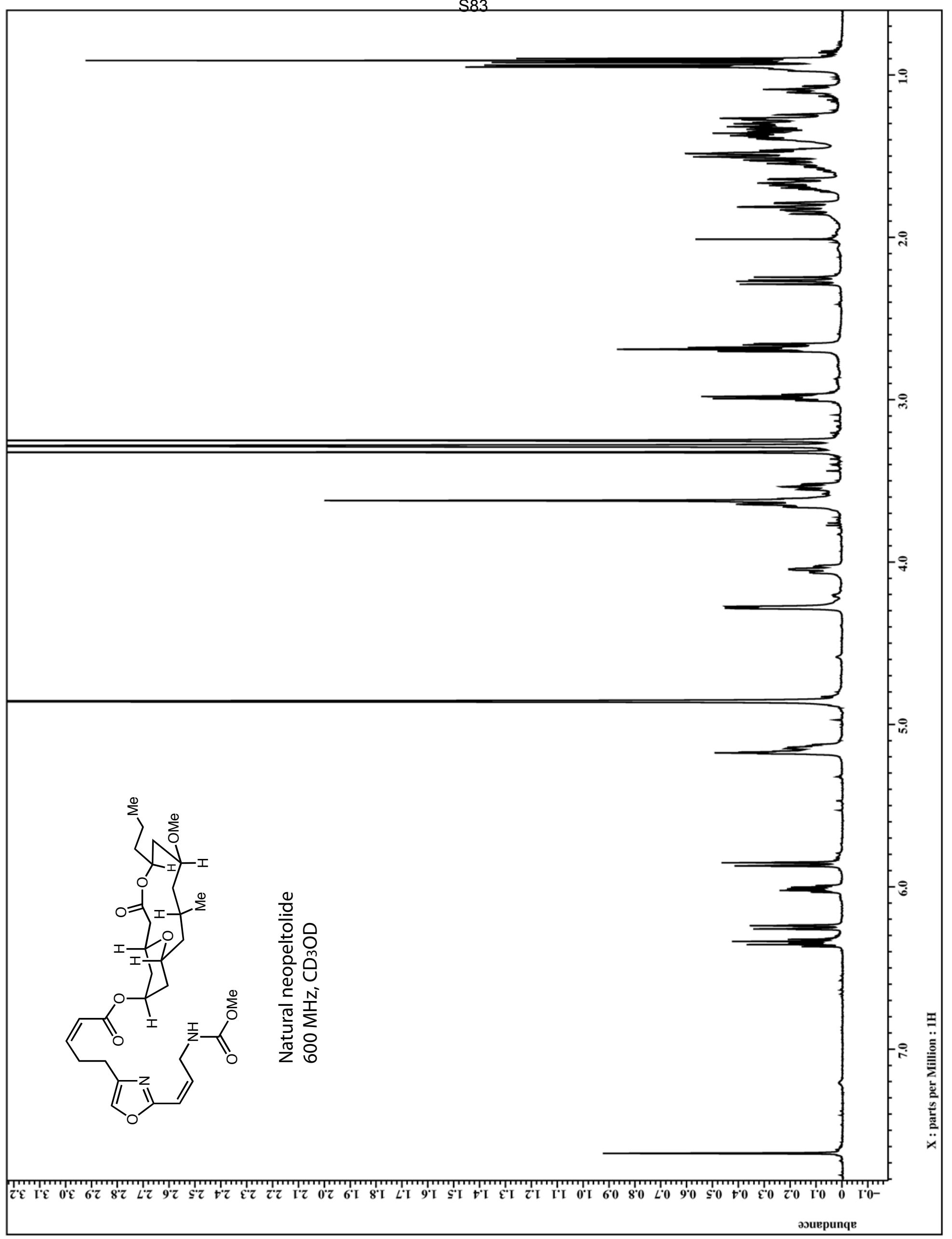




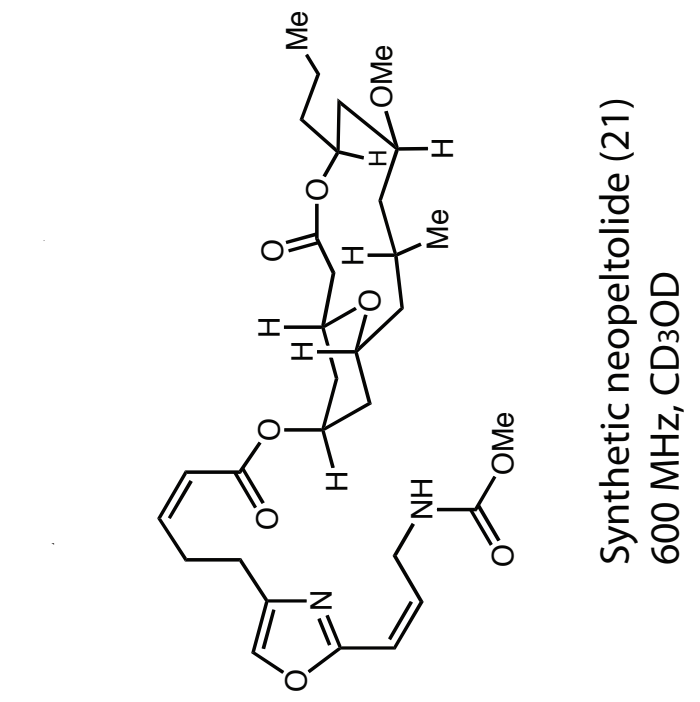

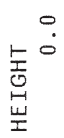

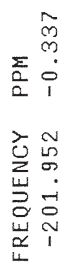

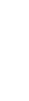

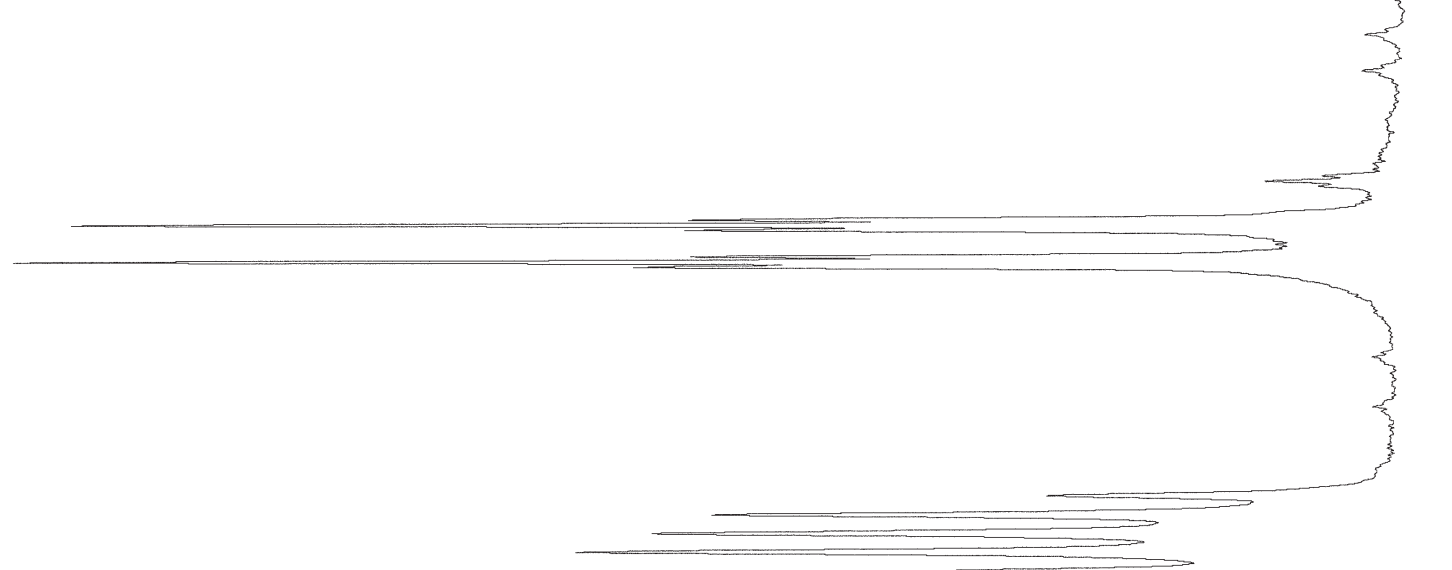

㞾-1

。ํ.

5

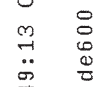

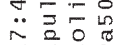

is

$\sim$ 蒠。

山。.

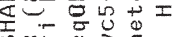

㟧势

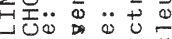

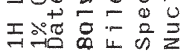




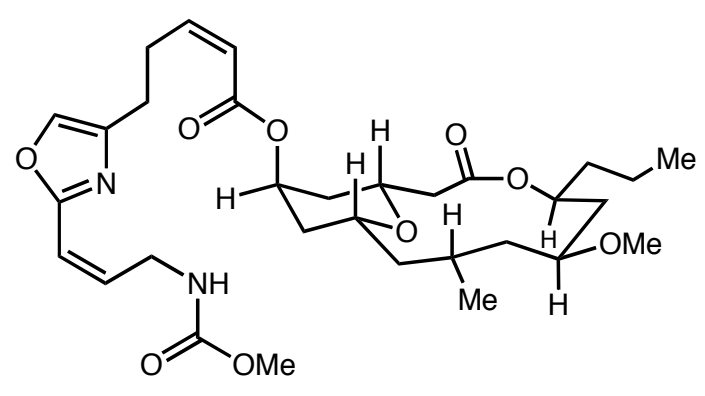

Natural neopeltolide

$600 \mathrm{MHz}, \mathrm{CD}_{3} \mathrm{OD}$ 


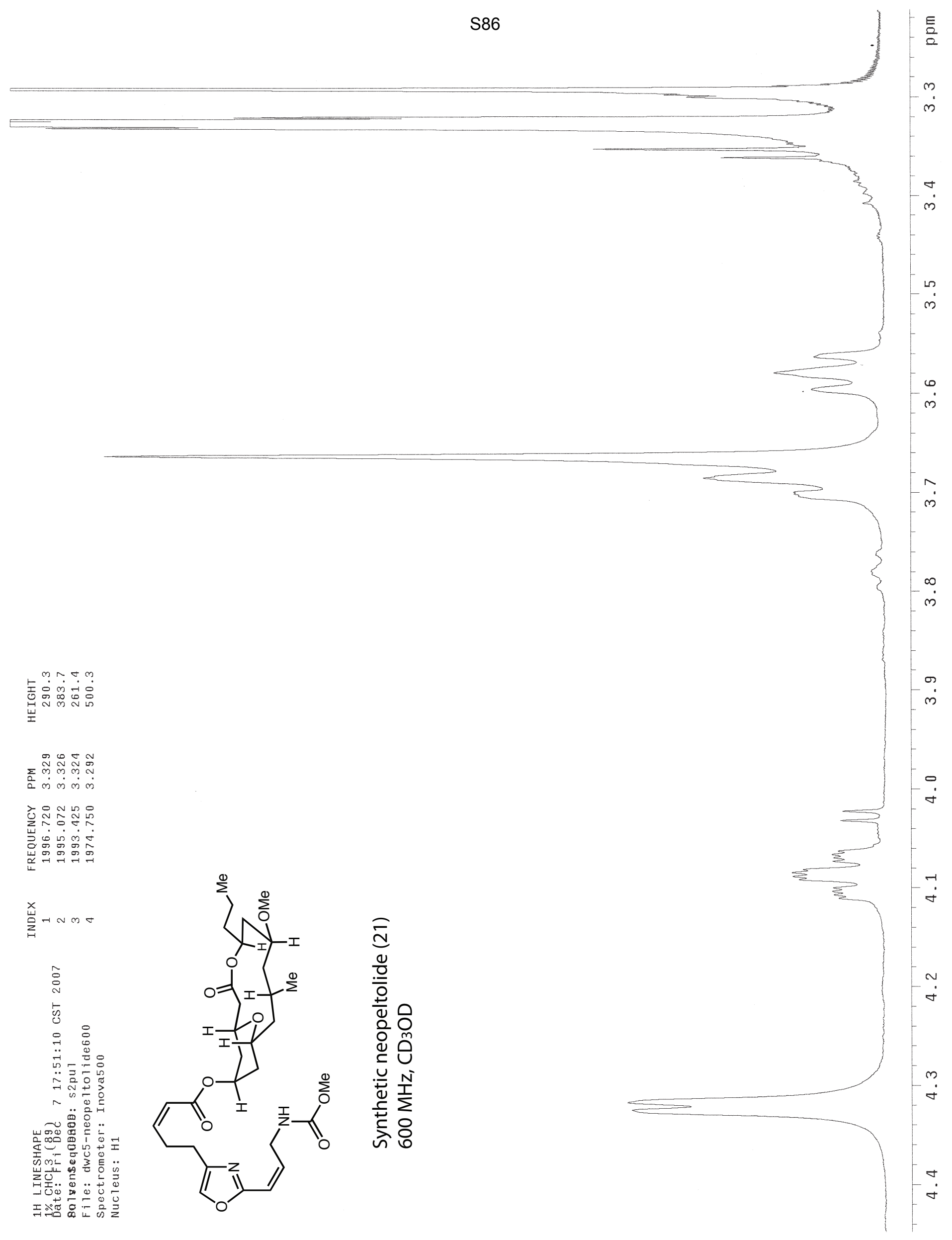




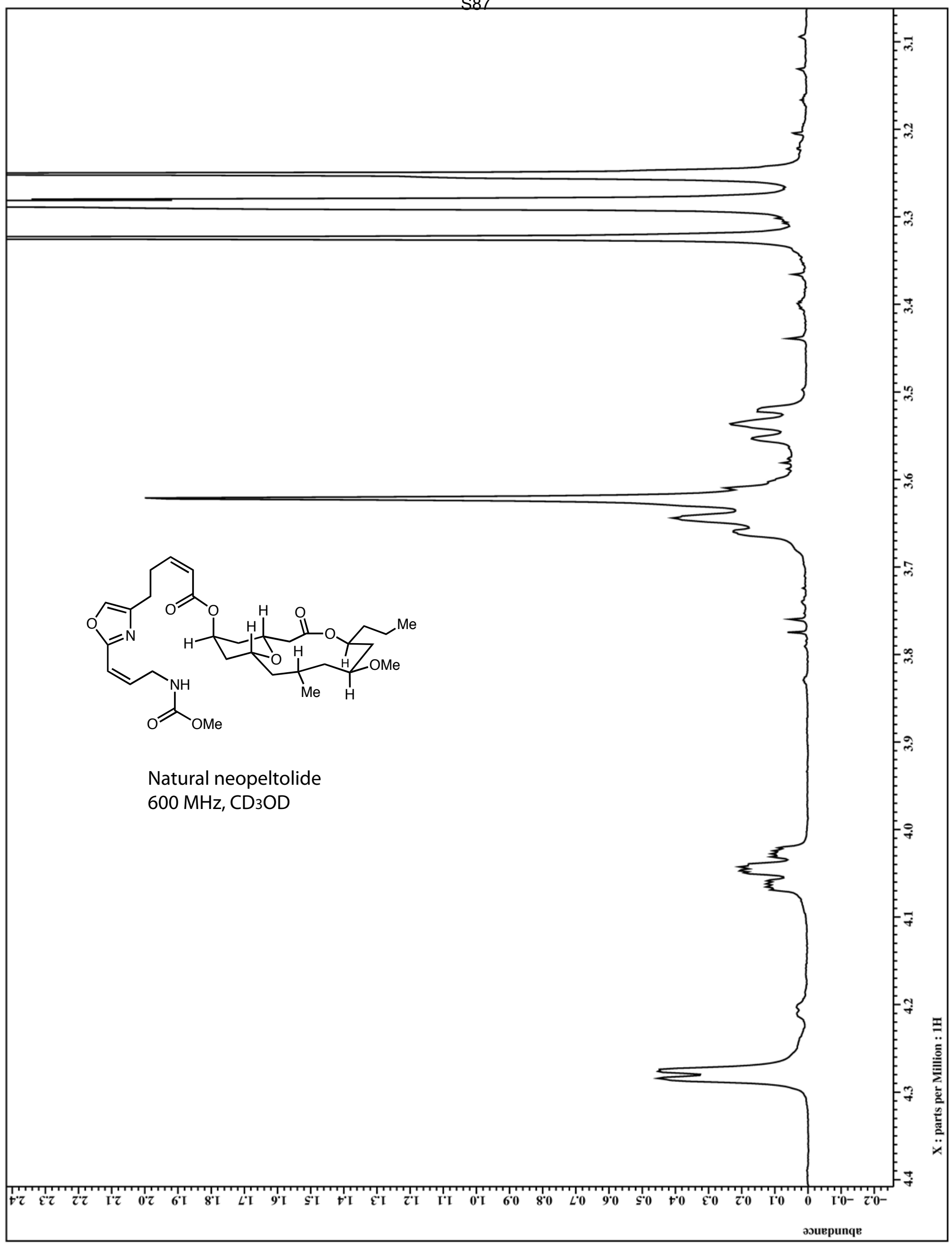


志递

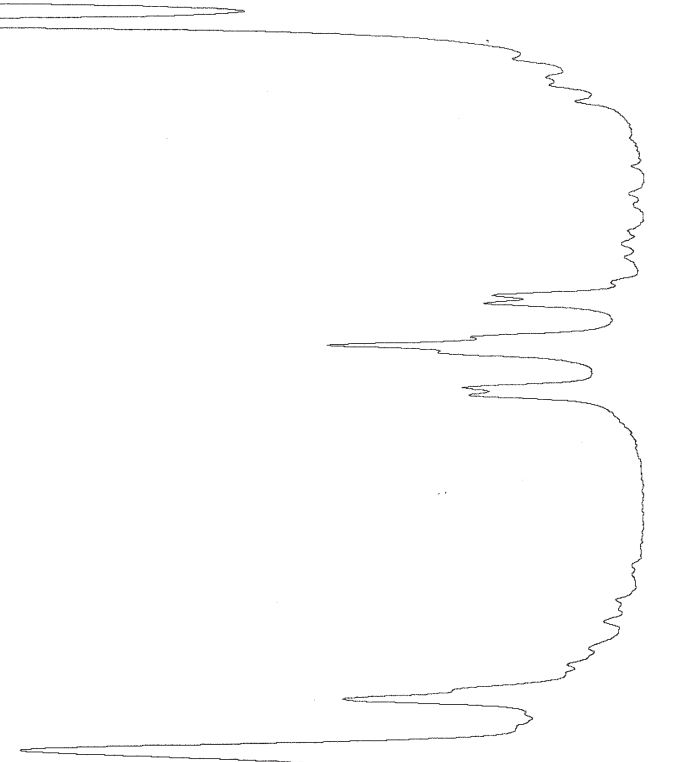

$\therefore$

$-1$

$\stackrel{n}{-1}$

-

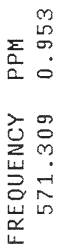

㞾-
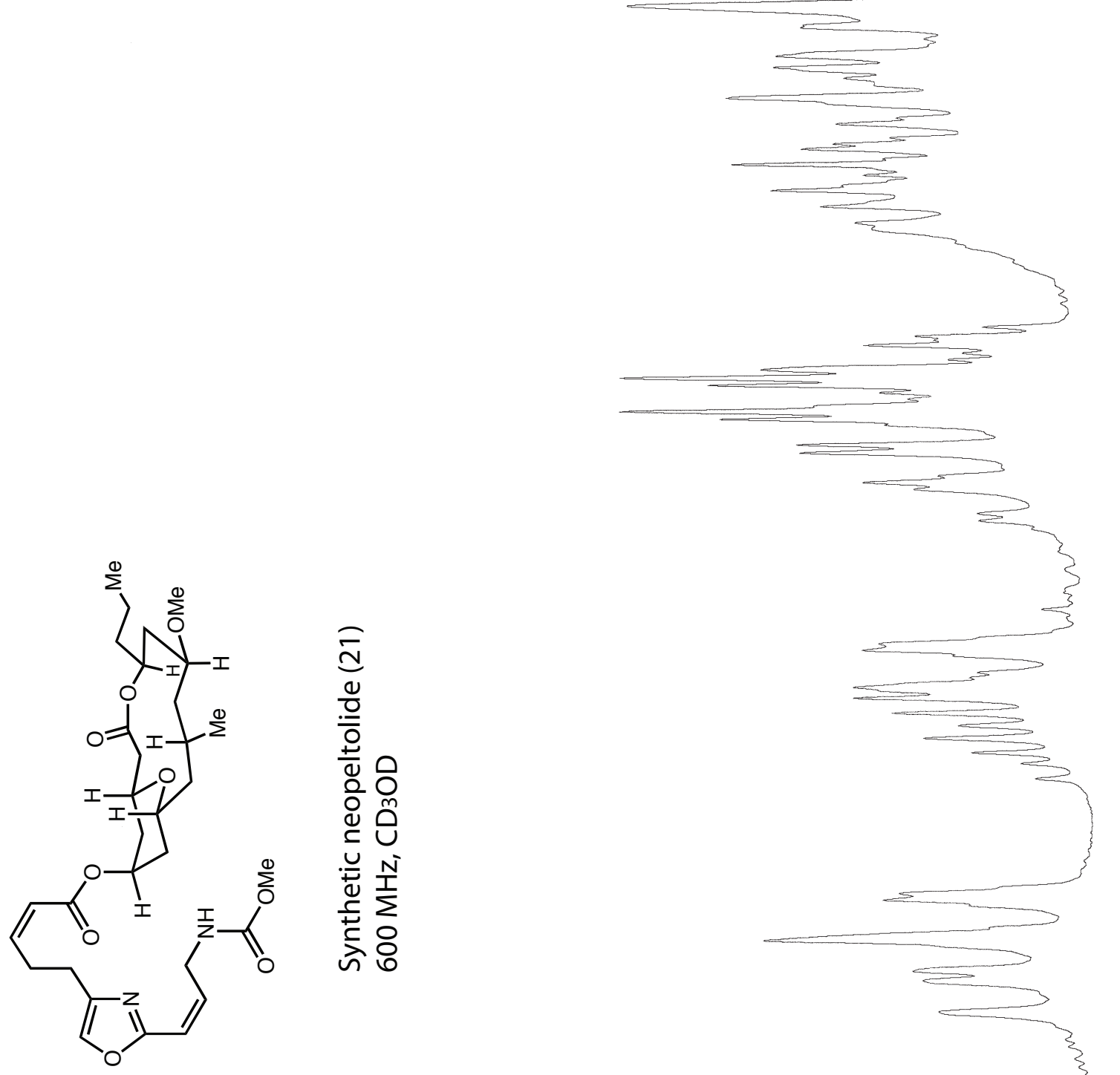

$\stackrel{-}{-}$
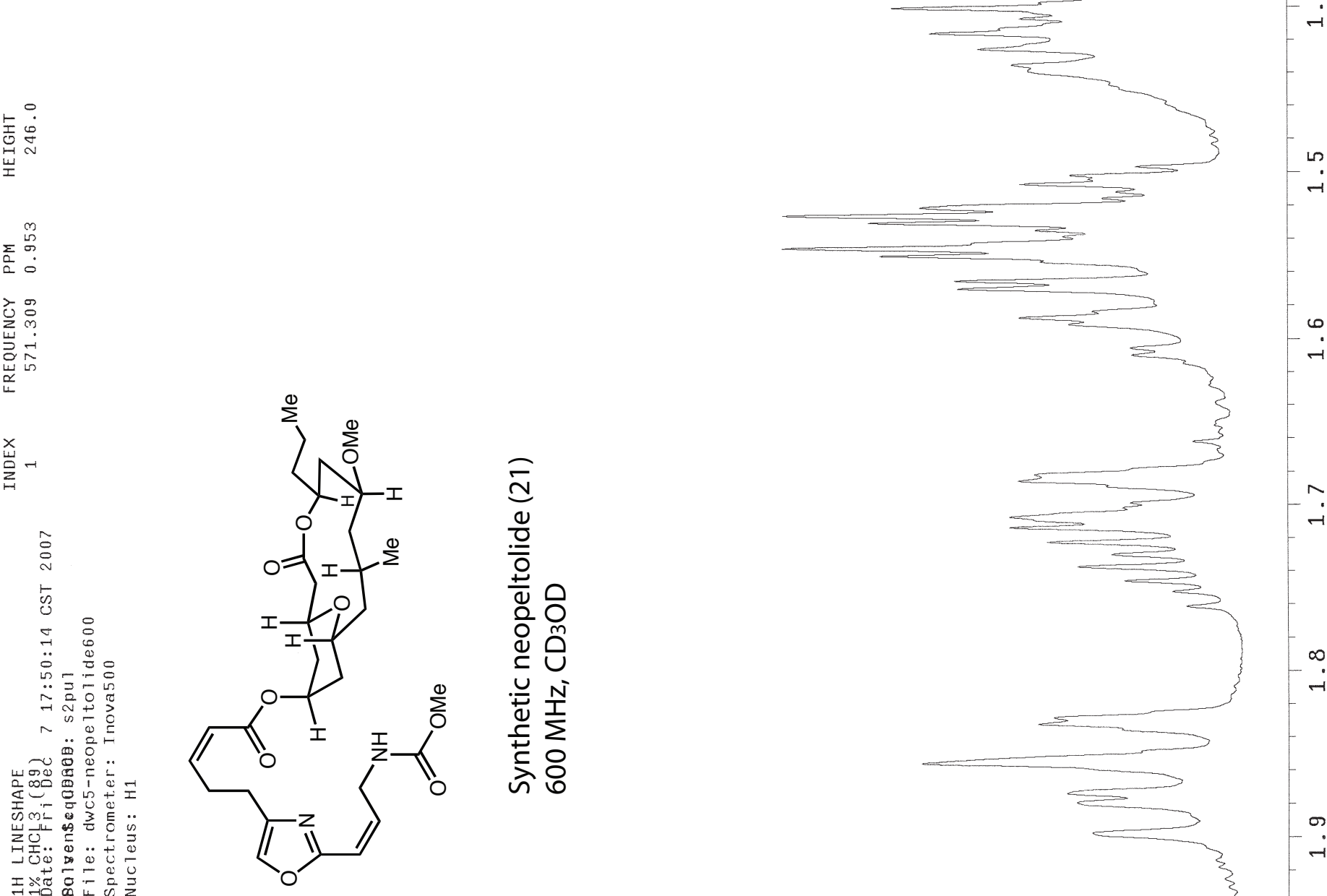

ปิ
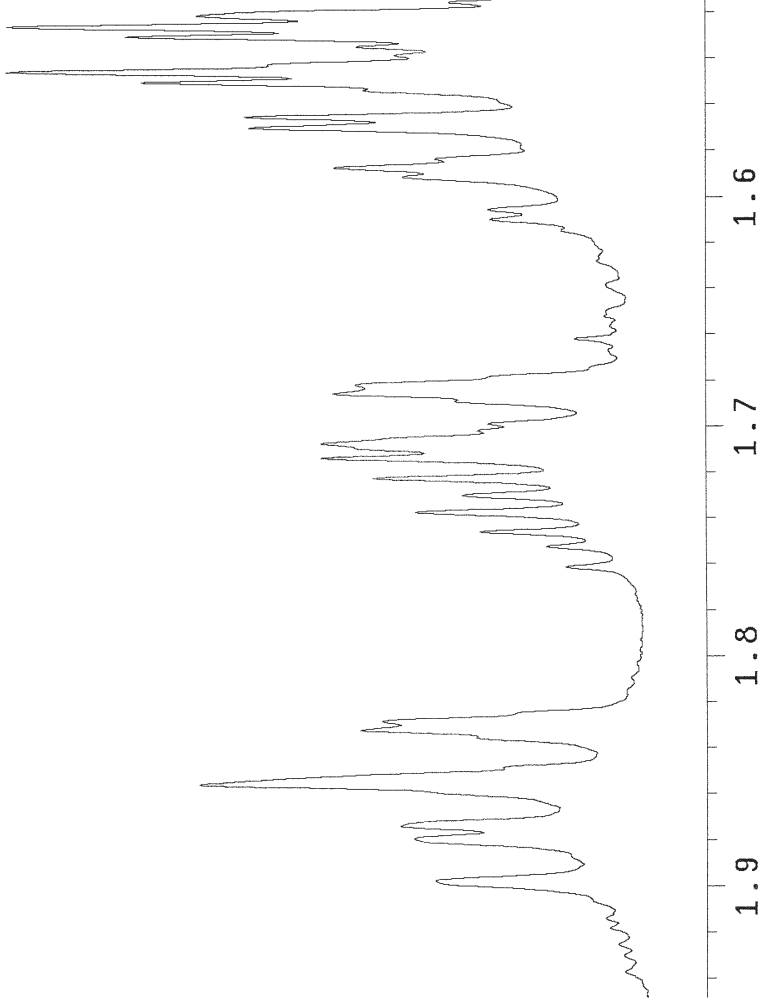


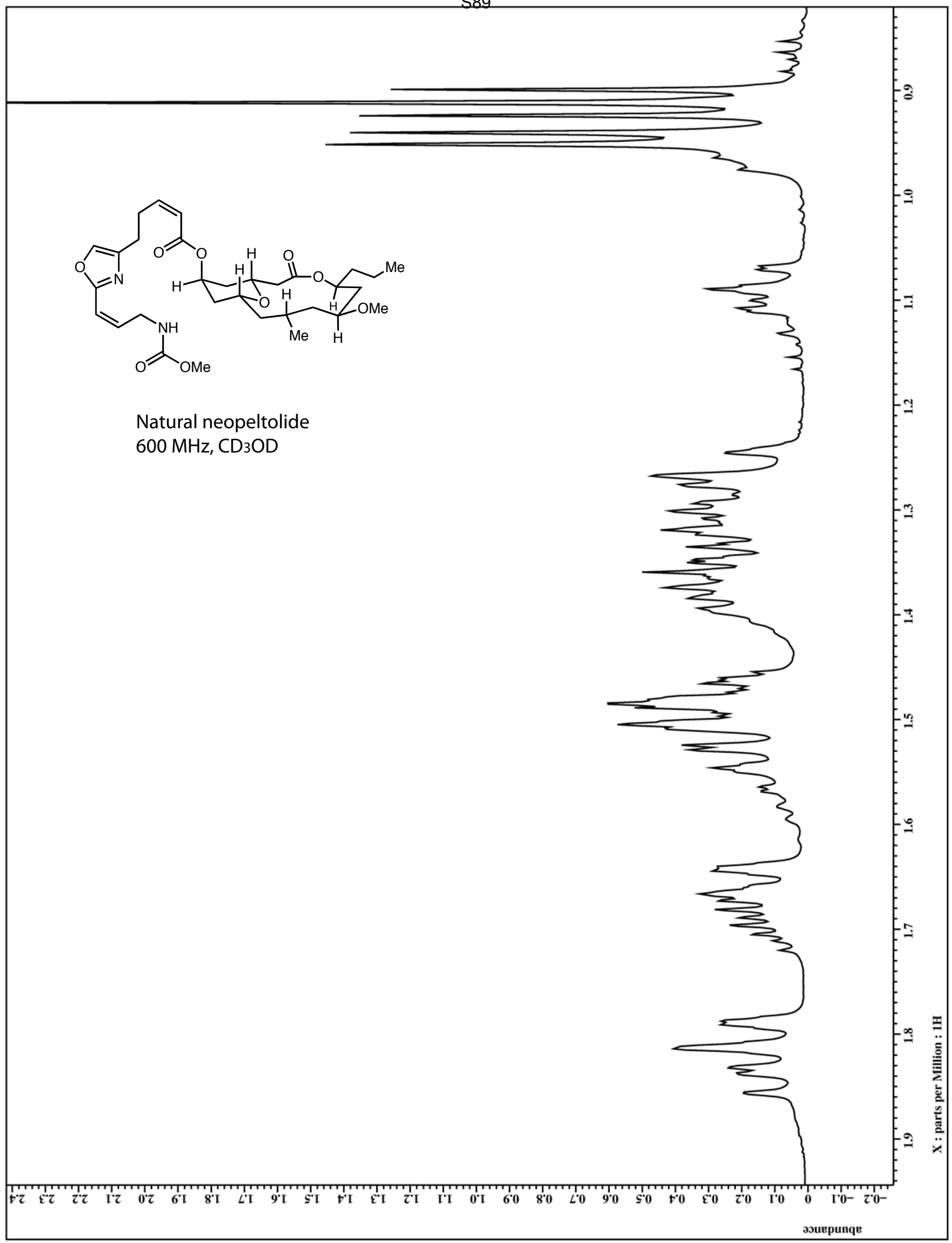




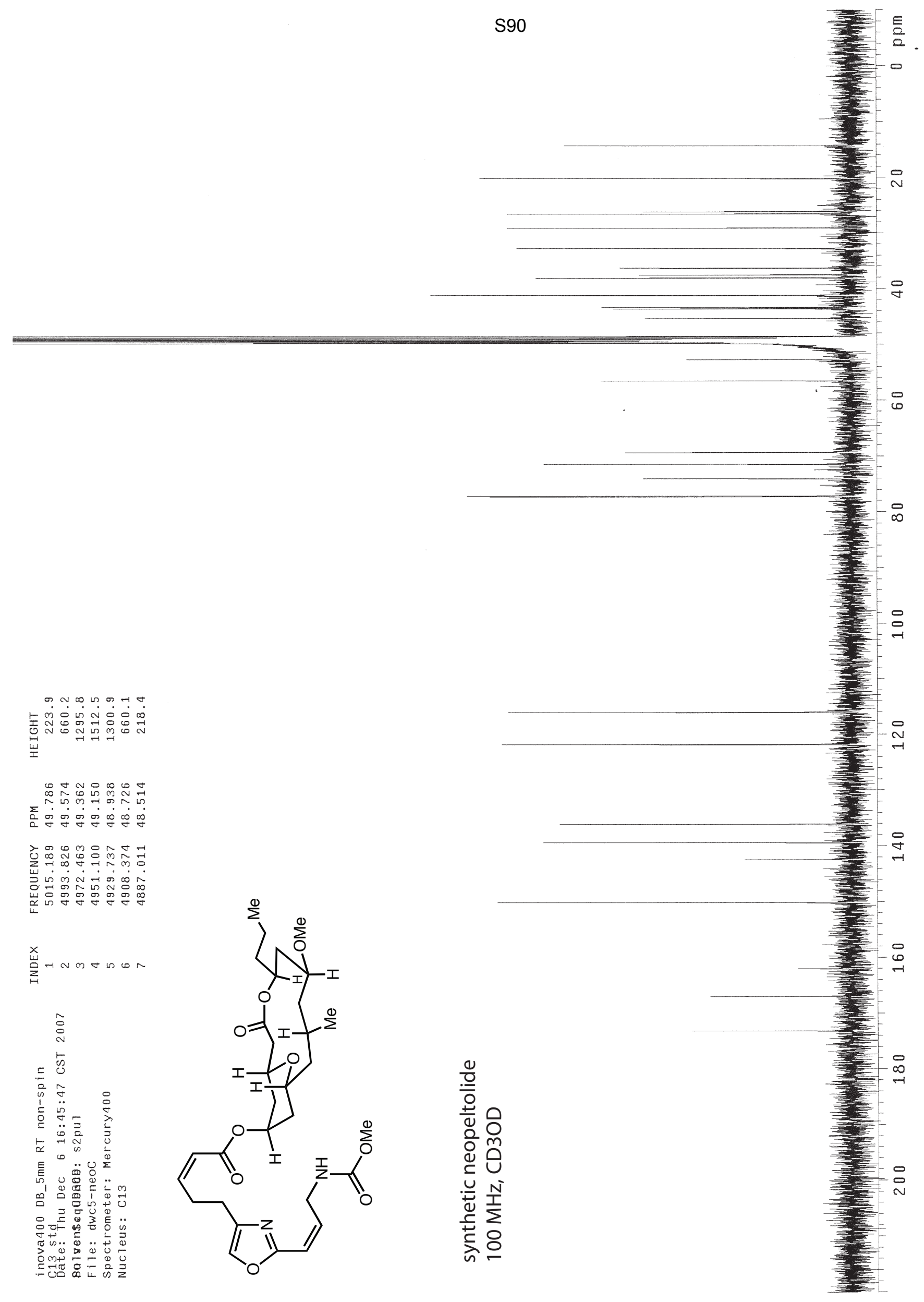




$$
1
$$




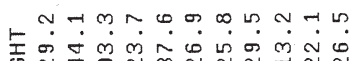

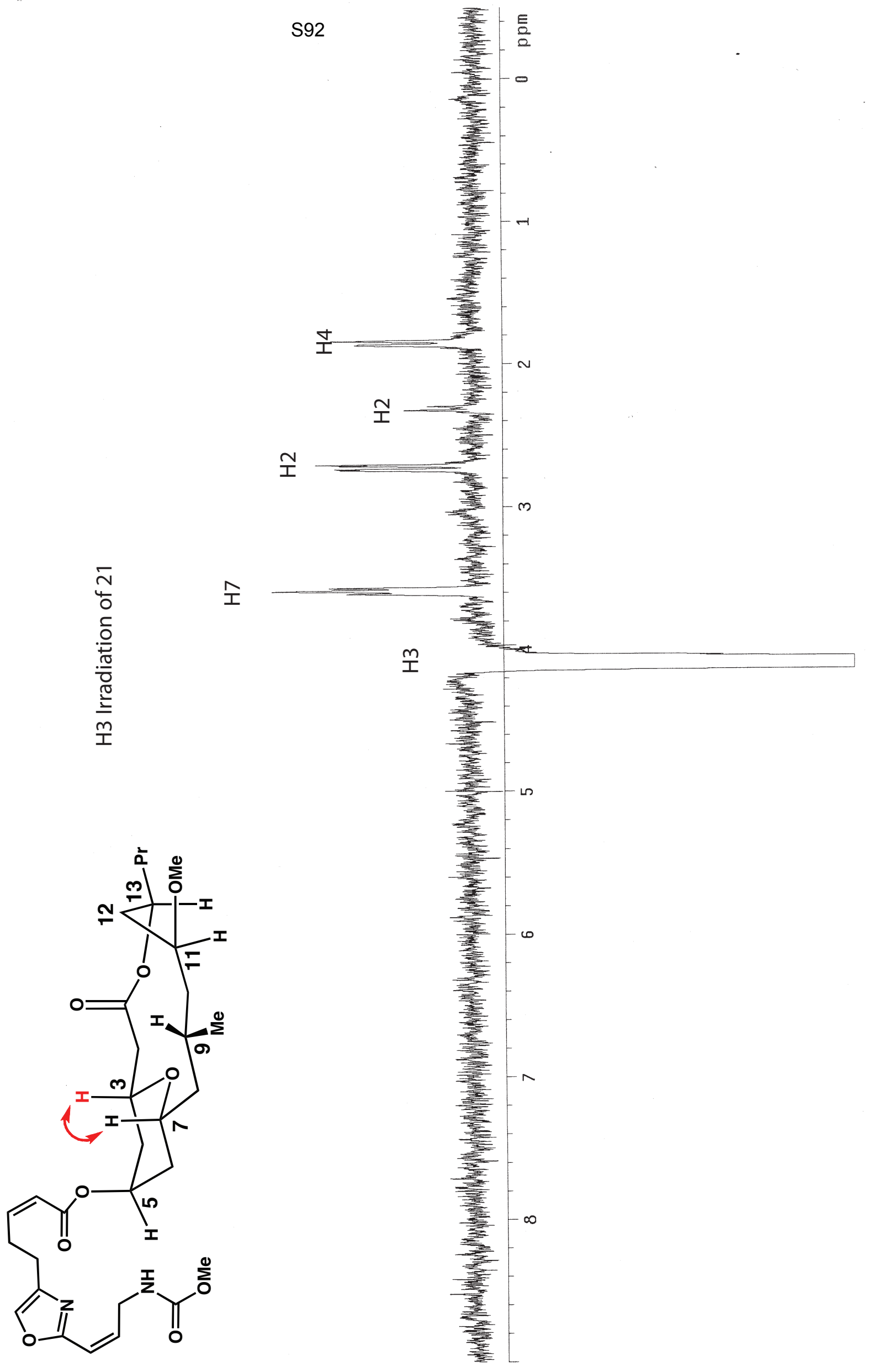




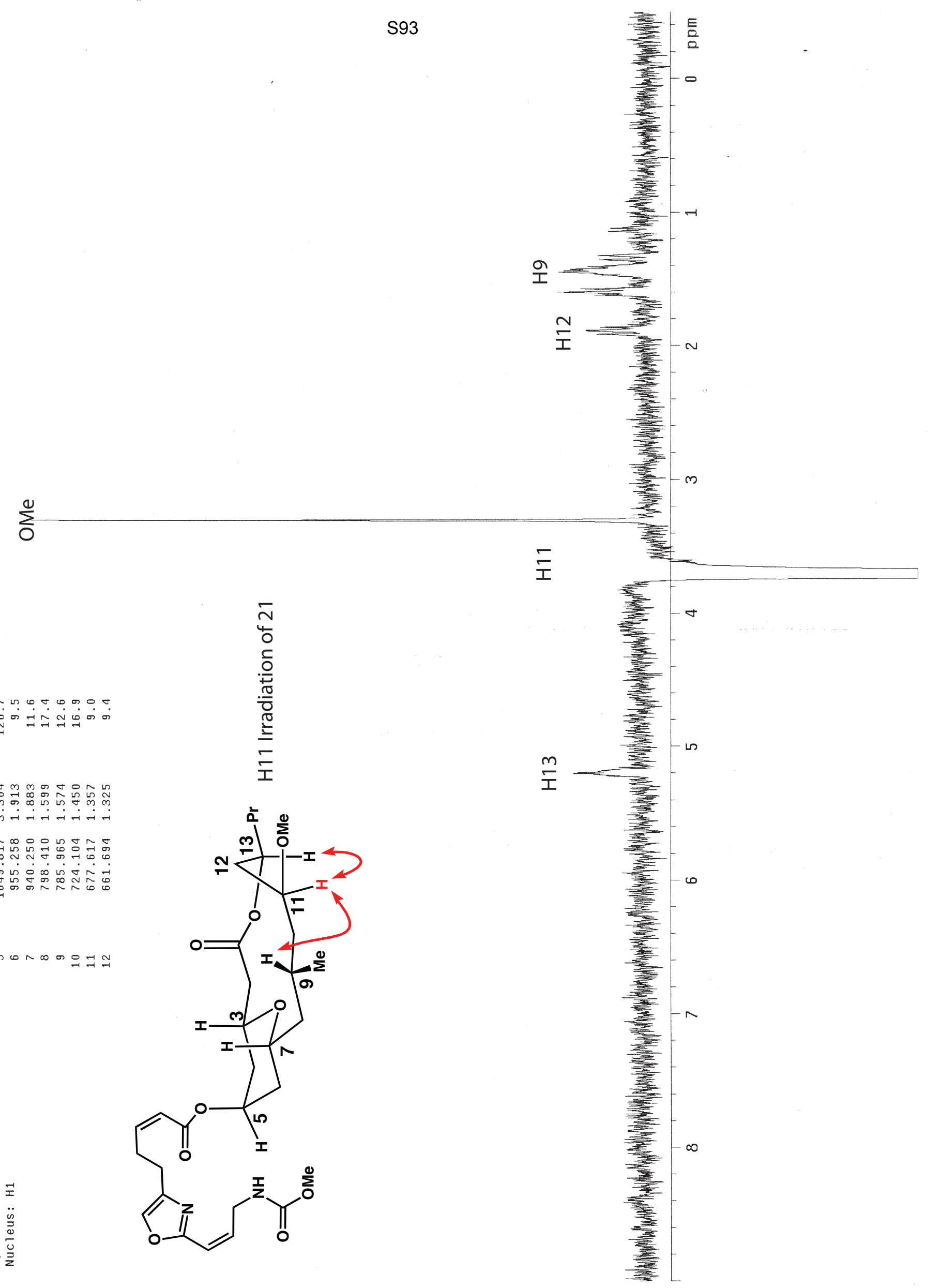

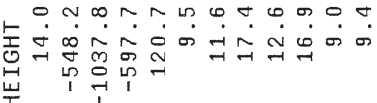

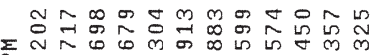

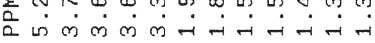

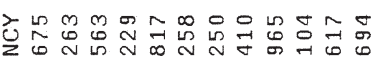

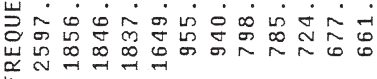

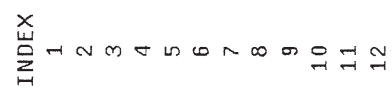

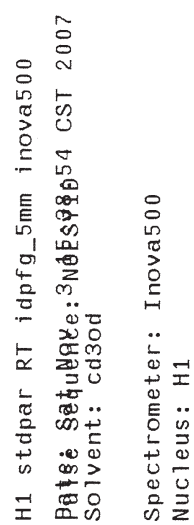

壬 


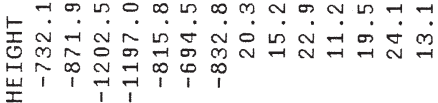

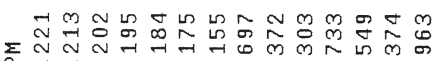

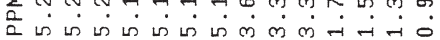

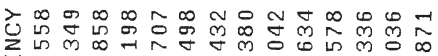

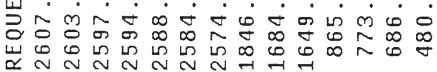

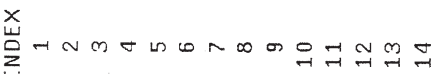

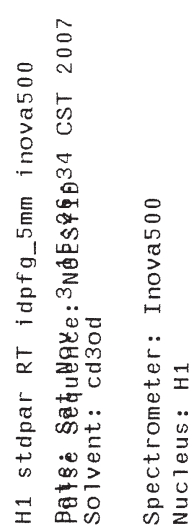

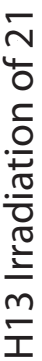

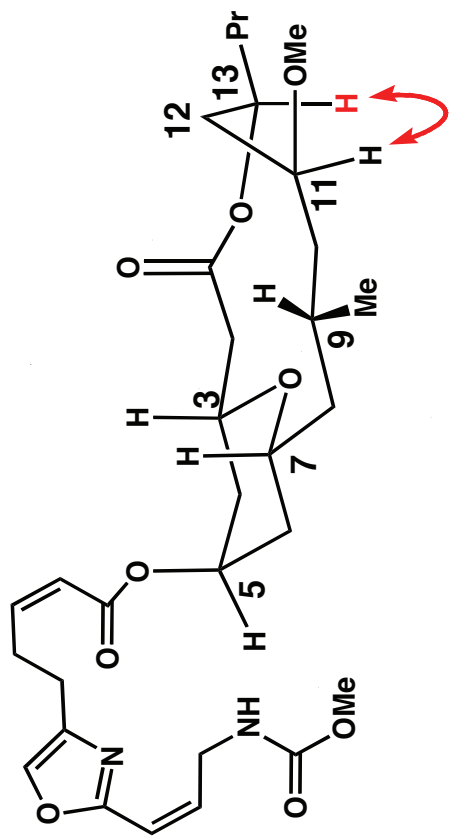

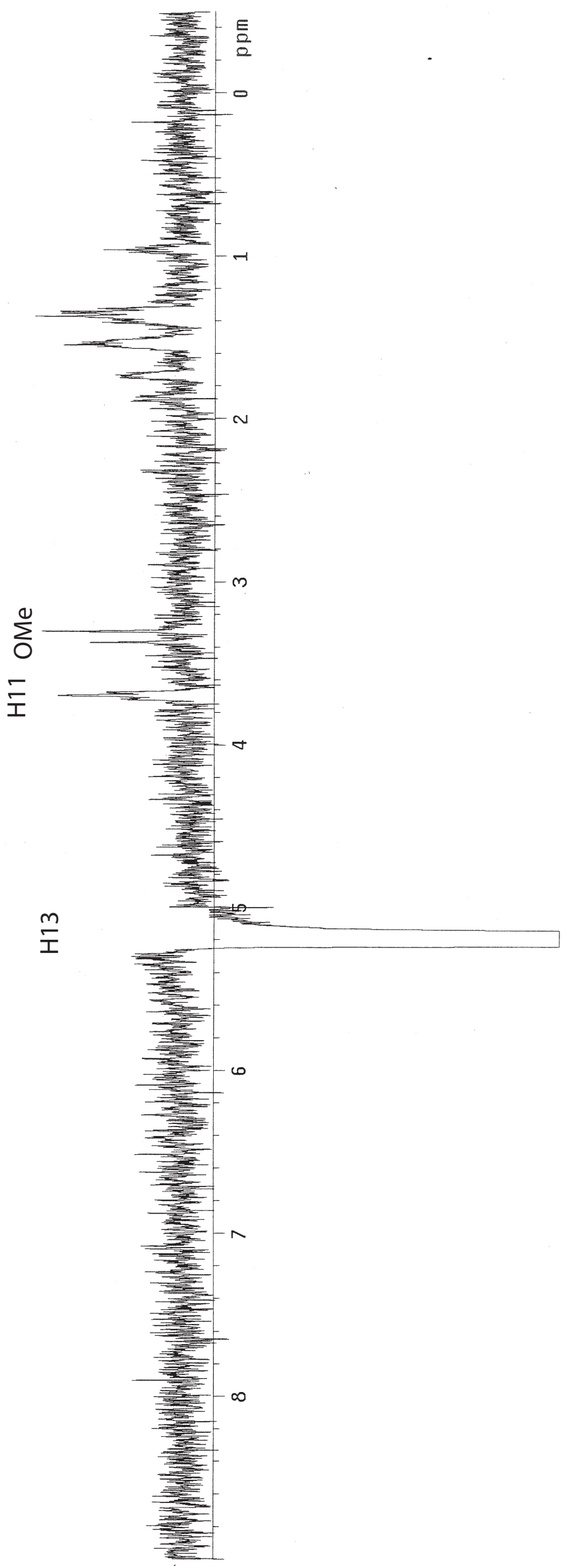




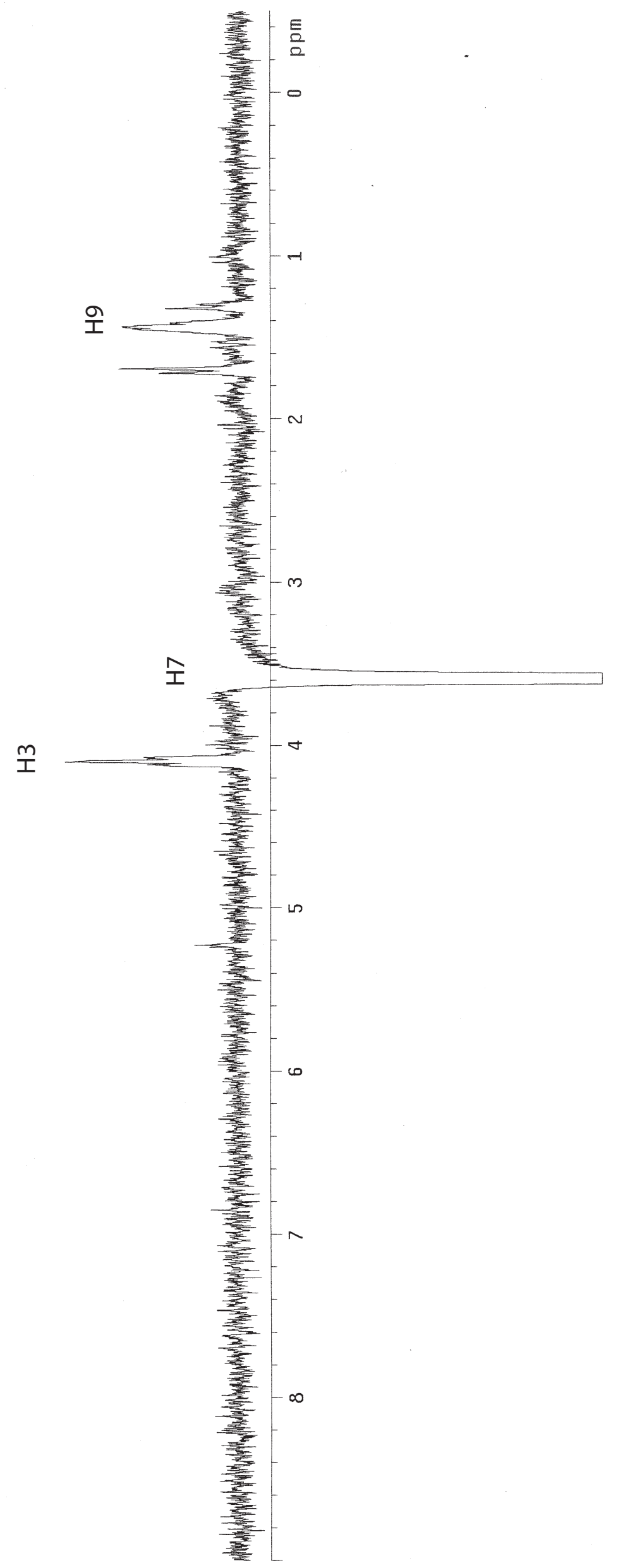

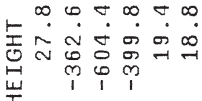

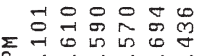

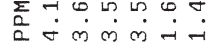

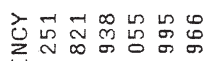

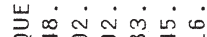

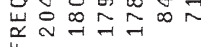

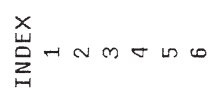
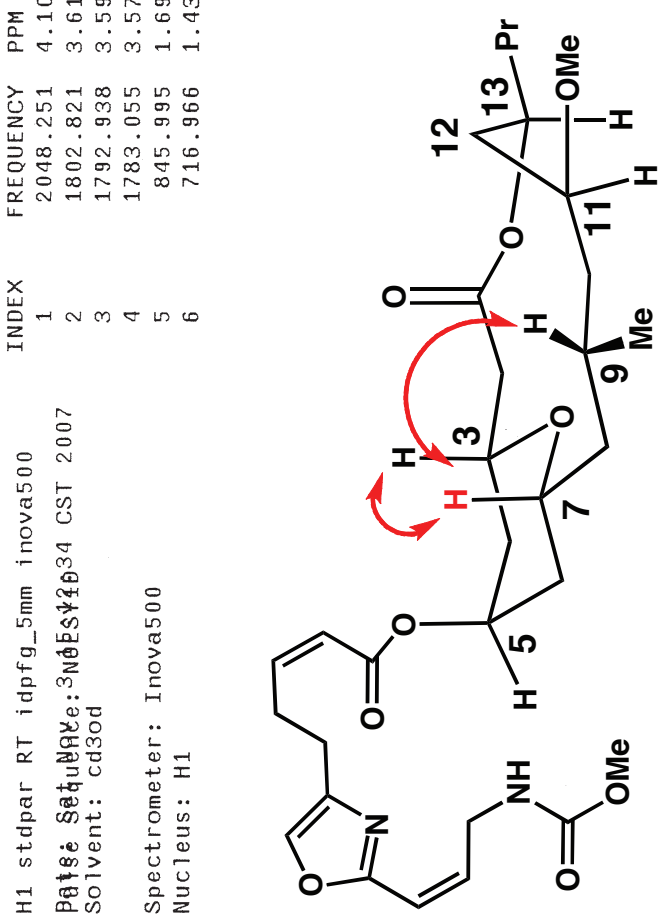

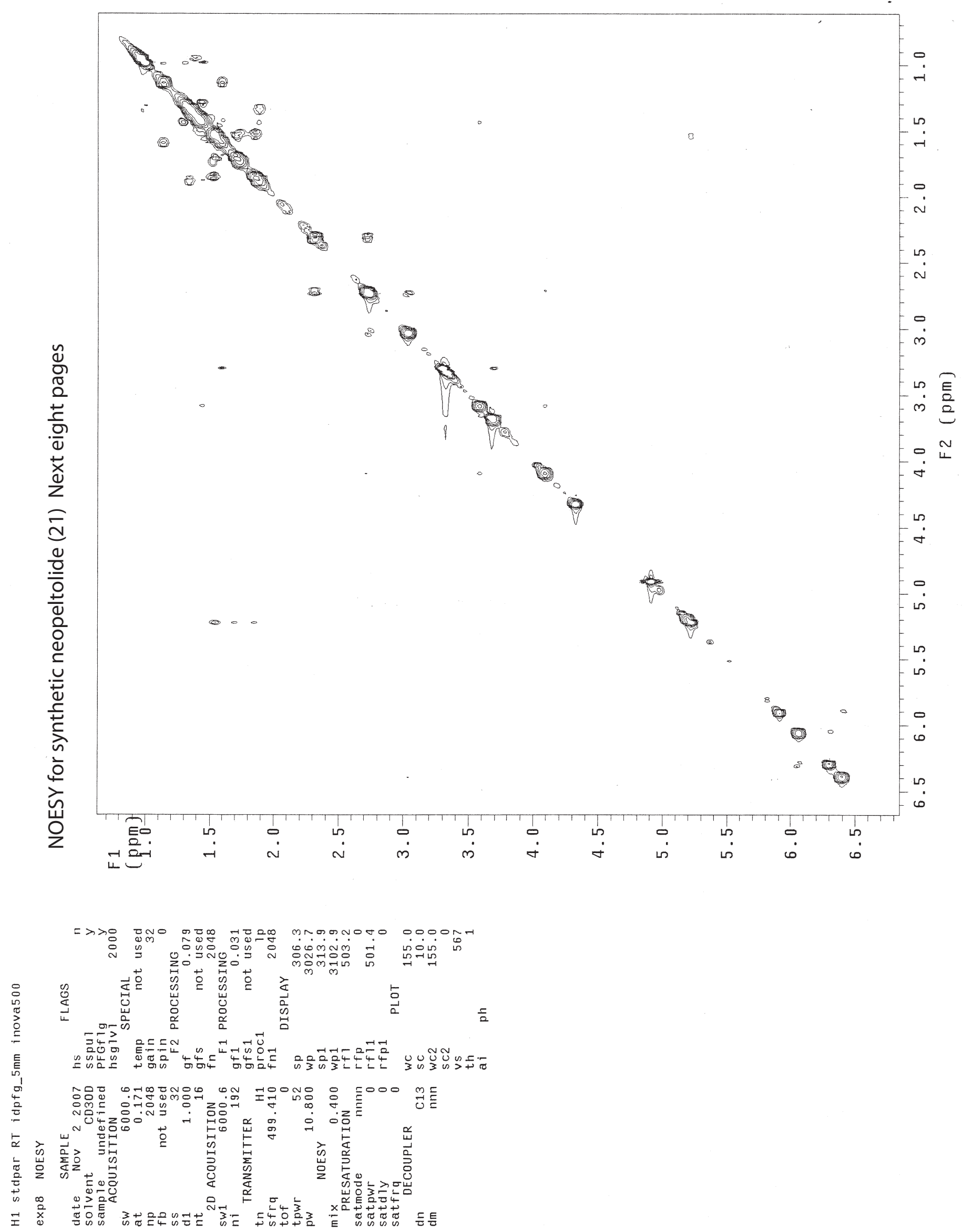

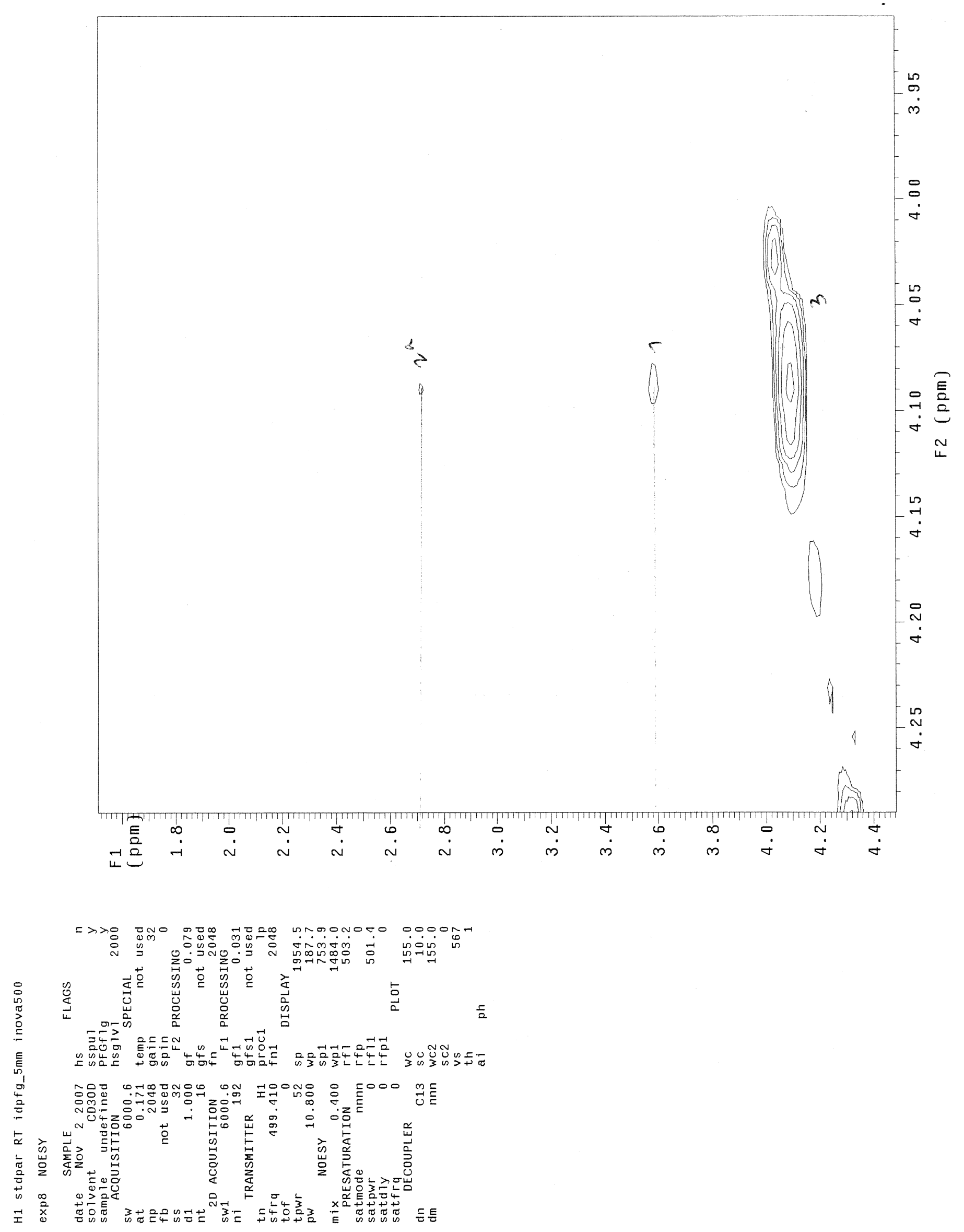

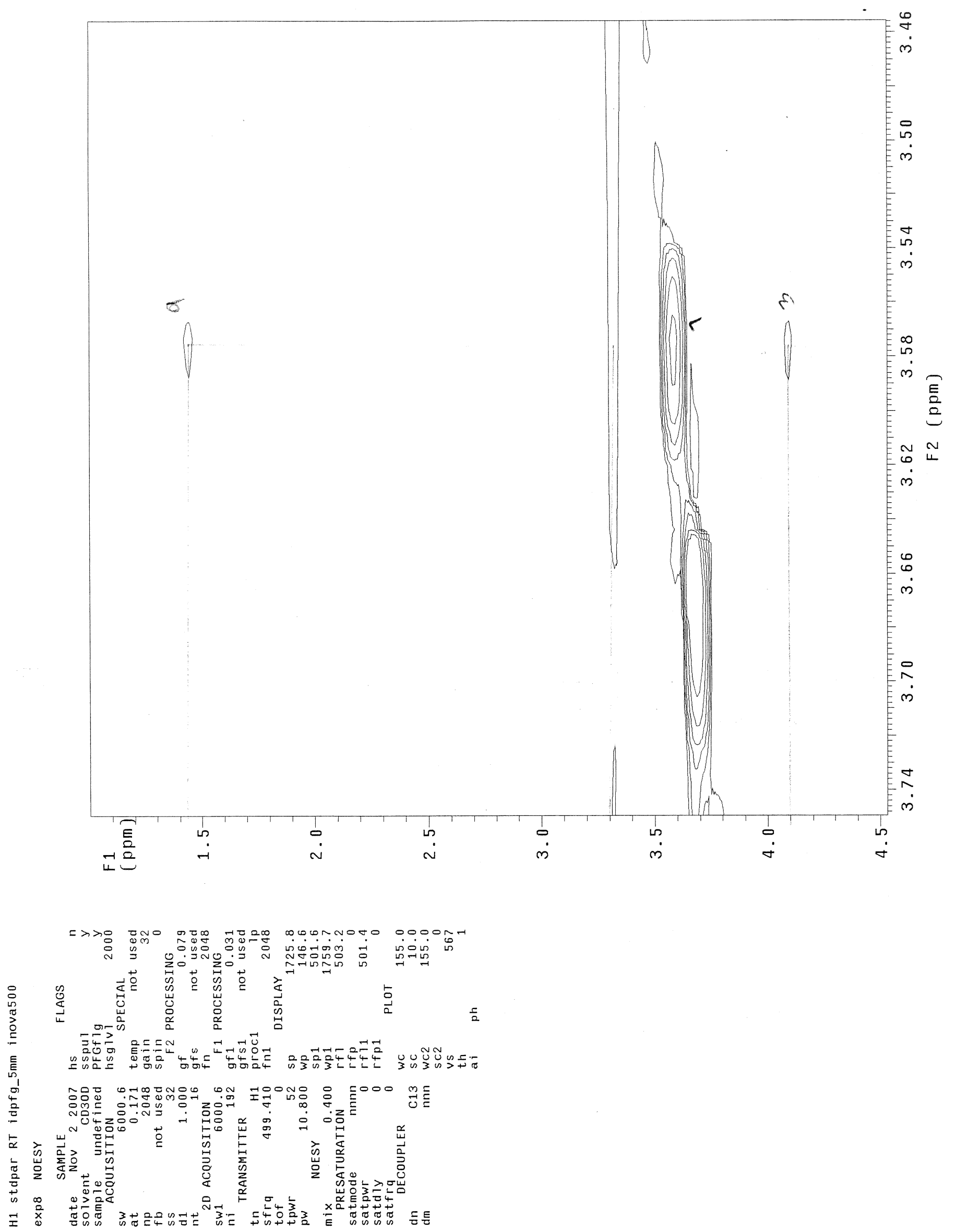

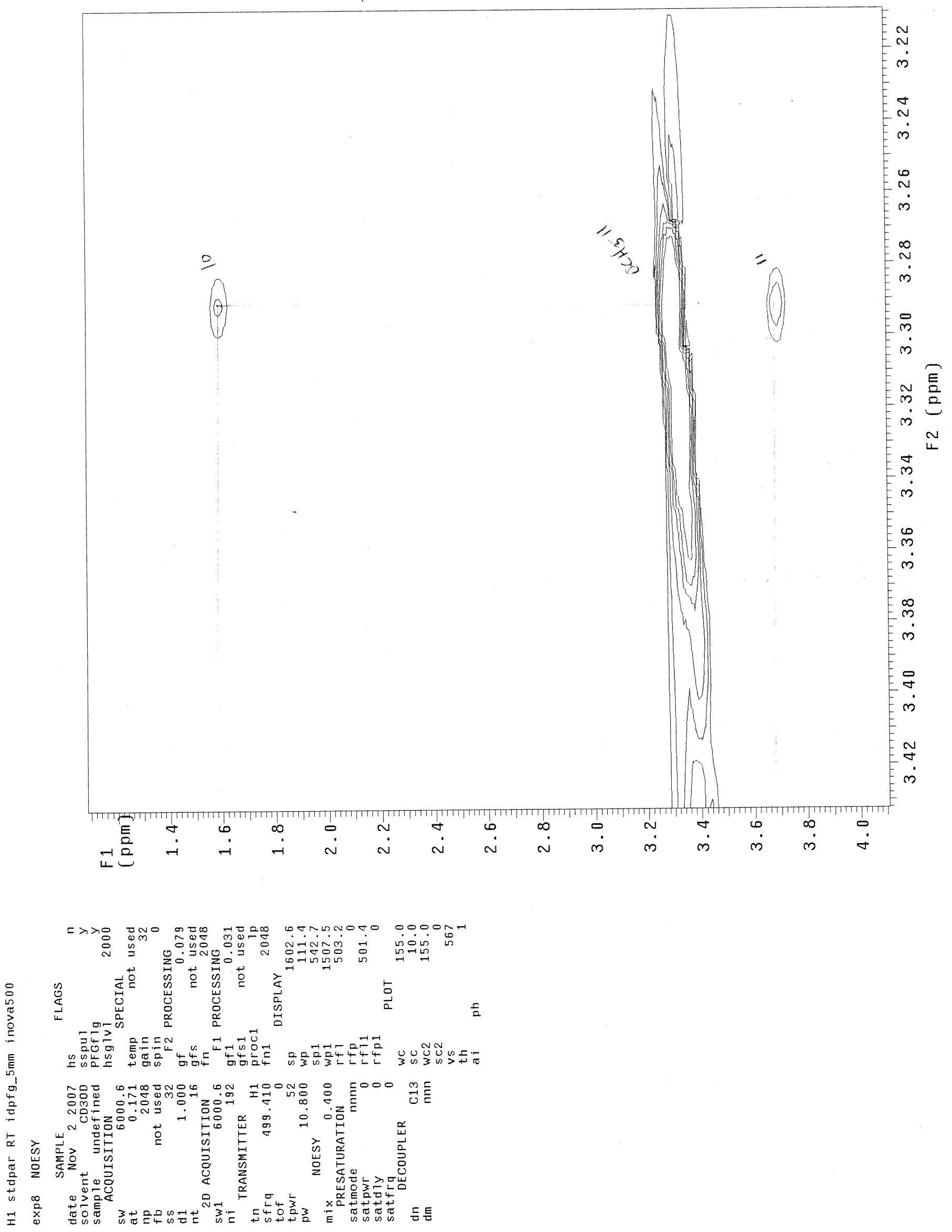
S100
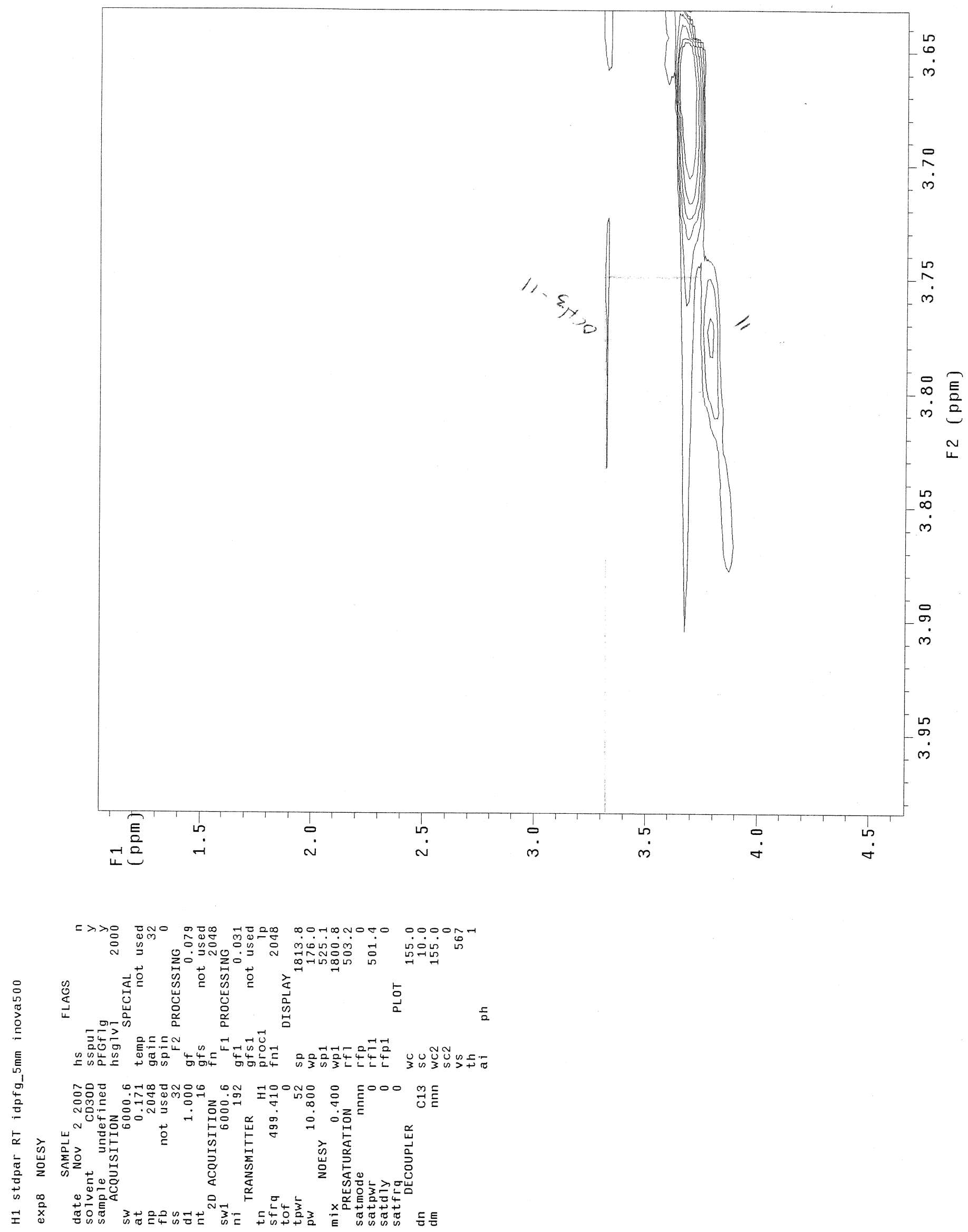
S101
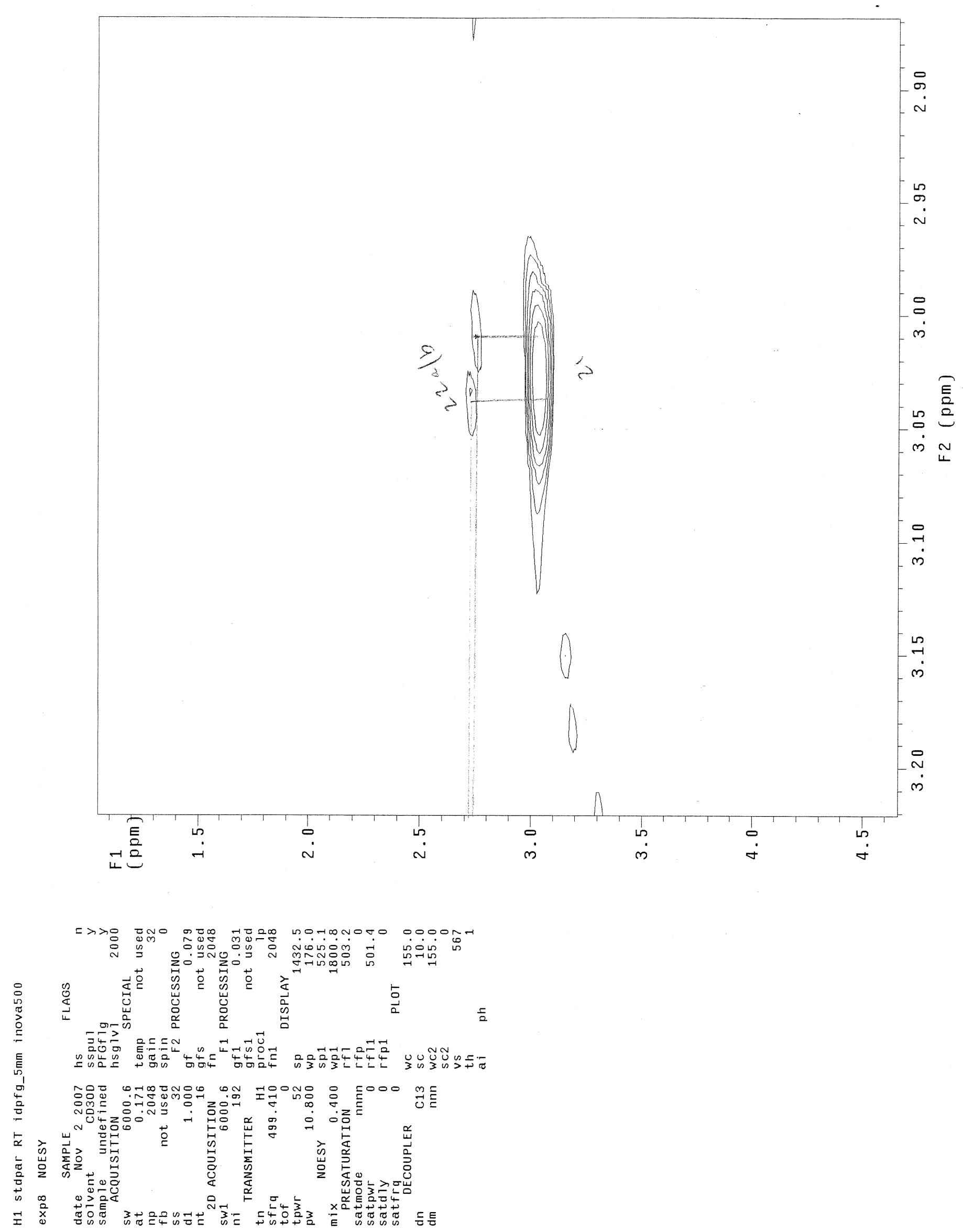
S102
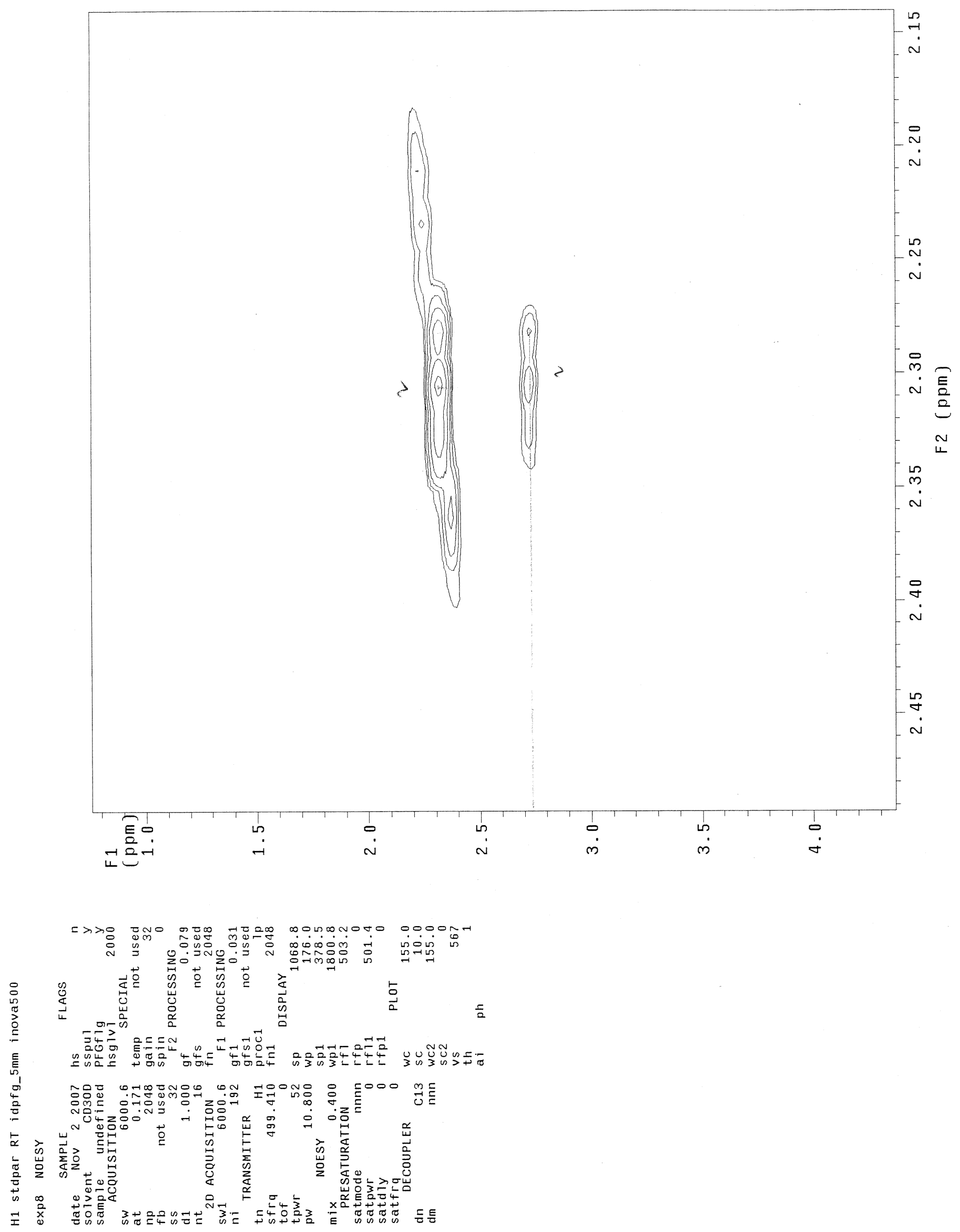

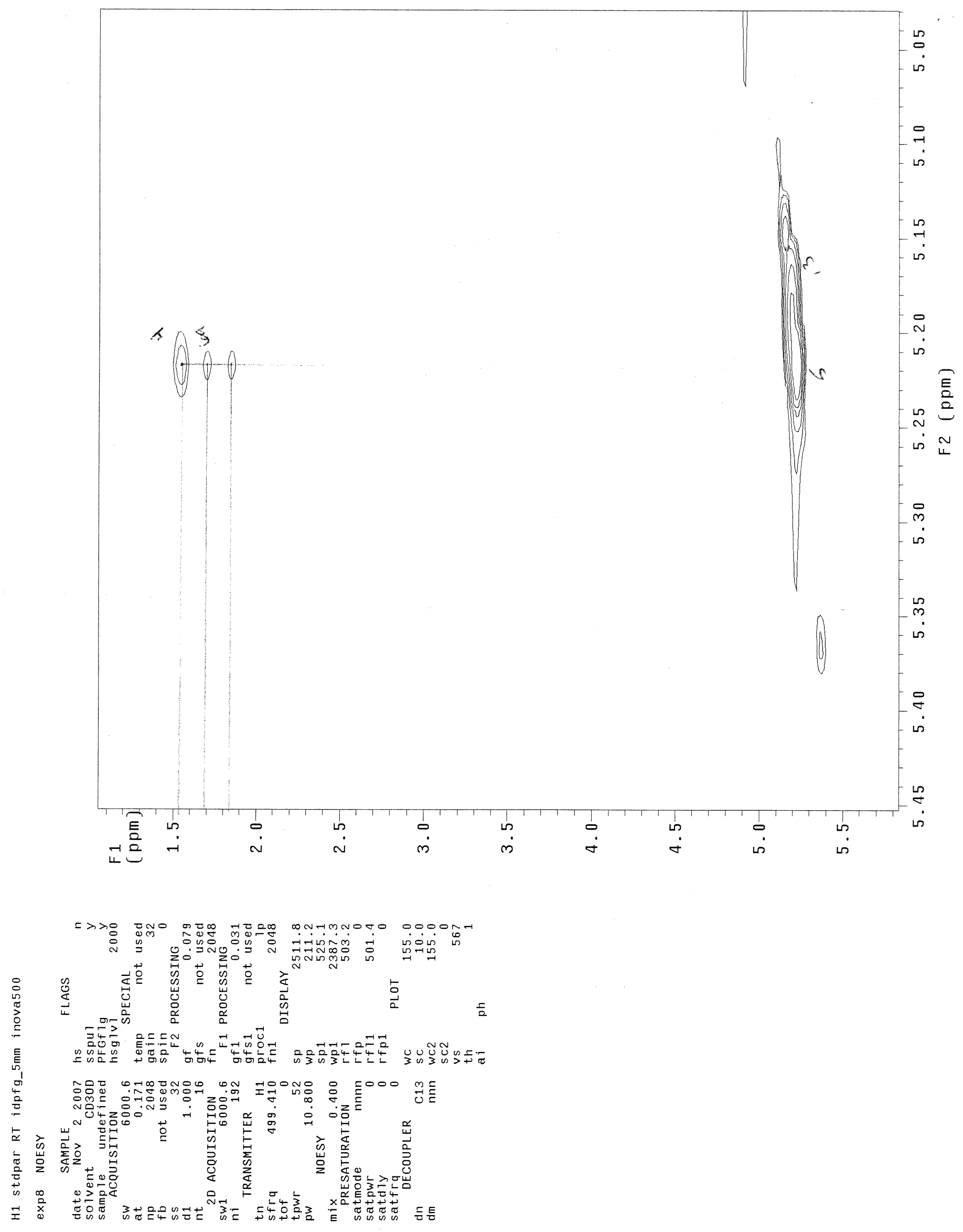
MS Formula Results: + Scan (0.360-0.457 min) - NeoPelt.d

\begin{tabular}{|c|c|c|c|c|c|c|c|c|c|}
\hline $\mathrm{Mz}$ & Species & Formula & Abundan & & & & & & \\
\hline 591.32772 & $(\mathrm{M}+\mathrm{H})+$ & $\mathrm{C} 31 \mathrm{H} 47 \mathrm{~N} 2 \mathrm{O}$ & 160 & 605 & & & & & \\
\hline Best & Formula & \begin{tabular}{l|l} 
Scor \\
\end{tabular} & Mass & Calc & c. Mass Diff & (ppm) & Abs Diff ( & (ppm) & $\overline{D B E}$ \\
\hline 四 & $\mathrm{C} 31 \mathrm{H} 46 \mathrm{~N} 2 \mathrm{O}$ & & \begin{tabular}{l|l}
00.32044 \\
\end{tabular} & 590 & 0.32033 & -0.18 & & 0.18 & 10 \\
\hline & Isotope & Abund \% & Calc Abund & & $\mathrm{m} / \mathrm{z}$ & Calcul & ated $\mathrm{m} / \mathrm{z}$ & Diff ( $p$ & pm) \\
\hline & 1 & 100 & & 100 & 591.32772 & & 591.32761 & & 0.18 \\
\hline & 2 & 29.13 & & 5.14 & 592.33123 & & 592.33088 & & 0.58 \\
\hline & 3 & 4.74 & & 7.84 & 593.33173 & & 593,33362 & & 3.18 \\
\hline & 4 & 0.67 & & 1.31 & 594.33161 & & 594.33629 & & 7.88 \\
\hline
\end{tabular}

\begin{tabular}{|c|c|c|c|c|c|c|c|c|c|c|}
\hline \multirow{2}{*}{$\begin{array}{c}\text { Best } \\
\square\end{array}$} & \multicolumn{2}{|c|}{ Formula } & \multicolumn{2}{|c|}{ Score } & Mass & \multicolumn{2}{|c|}{ Calc. Mass Diff (ppm) } & \multicolumn{2}{|c|}{ Abs Diff (ppm) } & \multirow[t]{2}{*}{ DBE } \\
\hline & $\mathrm{C} 27 \mathrm{H} 50 \mathrm{~N} 2 \mathrm{C}$ & $10 \mathrm{Si}$ & & 69.47 & 590.32044 & 590.32347 & 5.14 & & 5.14 & \\
\hline & Isotope & Abun & & Calc & Abund $\%$ & $\mathrm{~m} / \mathbf{z}$ & Calculate & $\mathrm{d} / \mathrm{z}$ & Diff (ppm) & \\
\hline & 1 & & 00 & & 100 & 591.32772 & & 1.33075 & 5.13 & \\
\hline & 2 & & & & 35.98 & 592.33123 & & 22.3335 & 3.83 & \\
\hline & 3 & & 74 & & 11.59 & 593.33173 & & 3.33358 & 3.11 & \\
\hline & 4 & & 67 & & 2.62 & 594.33161 & & 4.33474 & 5.27 & \\
\hline
\end{tabular}

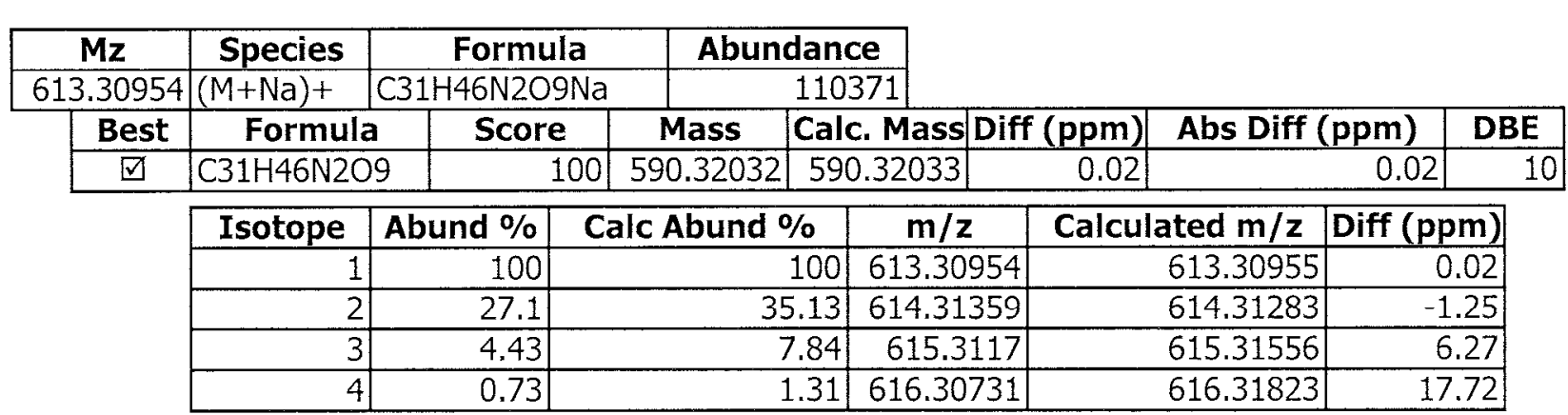

\begin{tabular}{|c|c|c|c|c|c|c|c|c|c|c|}
\hline \multirow{2}{*}{$\frac{\text { Best }}{\square}$} & \multicolumn{2}{|c|}{ Formula } & \multicolumn{2}{|c|}{ Score } & Mass & Calc. Mass & \multirow{2}{*}{\begin{tabular}{|r} 
Diff (ppm) \\
5.35
\end{tabular}} & \multicolumn{2}{|c|}{ Abs Diff (ppm) } & \multirow{2}{*}{$\frac{\mathrm{DBE}}{5}$} \\
\hline & C27H5ON2 & $10 \mathrm{Si}$ & & 71.25 & 590.32032 & 590.32347 & & & 5.35 & \\
\hline & Isotope & Abu & & Calc & Abund \% & $\mathrm{m} / \mathrm{z}$ & Calculate & $\mathrm{d} / \mathrm{z}$ & Diff (ppm) & \\
\hline & & & 100 & & 100 & 613.30954 & & 3.31269 & 5.14 & \\
\hline & 2 & & 7.1 & & 35.97 & 614.31359 & & 4.31544 & 3.01 & \\
\hline & 3 & & 4.43 & & 11.59 & 615.3117 & & 5.31552 & 6.21 & \\
\hline & & & 0.73 & & 2.62 & 616.30731 & & 5.31668 & 15.2 & \\
\hline
\end{tabular}




\section{Plot Window Report}

+ Scan (0.360-0.457 min, 7 scans) NeoPelt.d

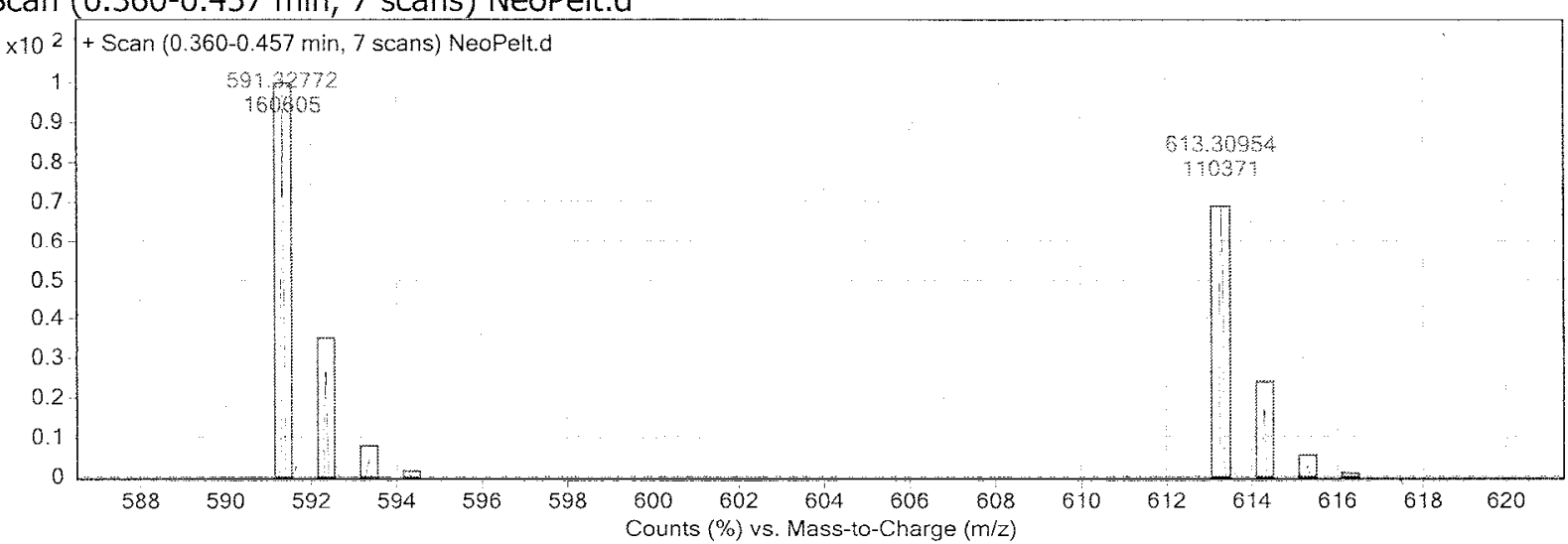


S106

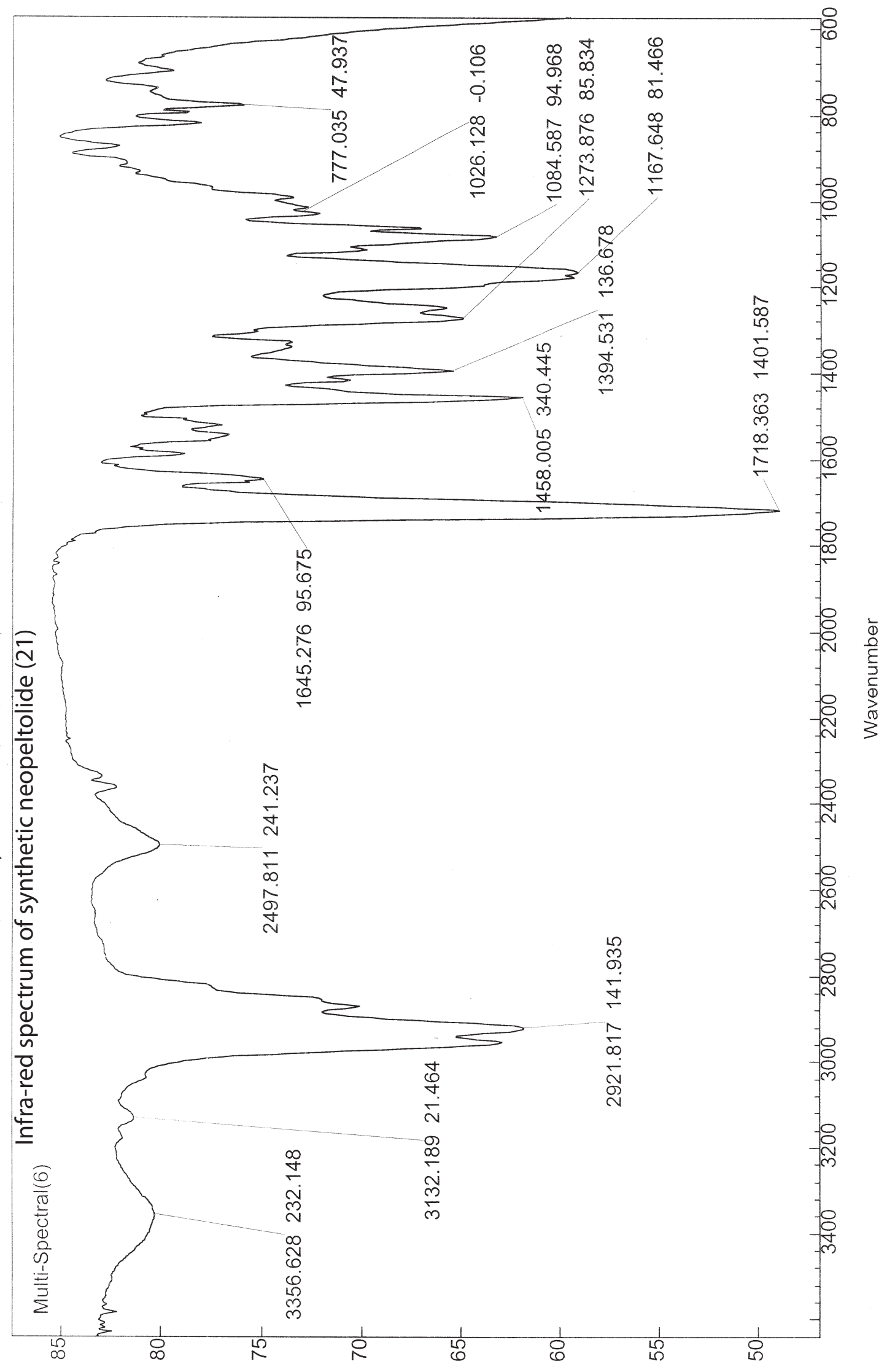

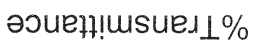

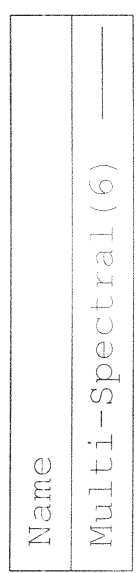

